This electronic thesis or dissertation has been

downloaded from the King's Research Portal at

https://kclpure.kcl.ac.uk/portal/

\title{
TOPICS IN OPTIMAL REINSURANCE DESIGN, RISK MEASURES, AND FORWARD
} PERFORMANCE PROCESSES

\section{Chong, Wing Fung}

Awarding institution:

King's College London

The copyright of this thesis rests with the author and no quotation from it or information derived from it may be published without proper acknowledgement.

\section{END USER LICENCE AGREEMENT}

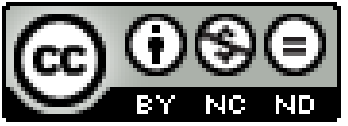

Unless another licence is stated on the immediately following page this work is licensed

under a Creative Commons Attribution-NonCommercial-NoDerivatives 4.0 International

licence. https://creativecommons.org/licenses/by-nc-nd/4.0/

You are free to copy, distribute and transmit the work

Under the following conditions:

- Attribution: You must attribute the work in the manner specified by the author (but not in any way that suggests that they endorse you or your use of the work).

- $\quad$ Non Commercial: You may not use this work for commercial purposes.

- $\quad$ No Derivative Works - You may not alter, transform, or build upon this work.

Any of these conditions can be waived if you receive permission from the author. Your fair dealings and other rights are in no way affected by the above.

Take down policy

If you believe that this document breaches copyright please contact librarypure@kcl.ac.uk providing details, and we will remove access to the work immediately and investigate your claim. 


\section{TOPICS IN OPTIMAL REINSURANCE DESIGN, RISK MEASURES, AND FORWARD PERFORMANCE PROCESSES \\ by \\ CHONG, WING FUNG \\ B.Sc., M.Phil. H.K.}

A thesis submitted in partial fulfilment of the requirements for the Degree of Joint Doctor of Philosophy at The University of Hong Kong and King's College London

August 2017 


\section{DECLARATION}

I declare that this thesis represents my own work under the supervision of Dr. Ka Chun Cheung and Dr. Gechun Liang during the period 2014-2017 for the degree of Joint Doctor of Philosophy at The University of Hong Kong and King's College London. The work submitted has not been previously included in any thesis, dissertation or report submitted to these Universities or to any other institution for a degree, diploma or other qualification.

The copyright of this thesis rests with the author and is made available under a Creative Commons Attribution Non-Commercial No Derivatives licence. Researchers are free to copy, distribute or transmit the thesis on the condition that they attribute it, that they do not use it for commercial purposes and that they do not alter, transform or build upon it. For any reuse or redistribution, researchers must make clear to others the licence terms of this work. 


\title{
Abstract of thesis entitled \\ TOPICS IN OPTIMAL REINSURANCE \\ DESIGN, RISK MEASURES, AND FORWARD PERFORMANCE PROCESSES
}

submitted by

\section{CHONG, WING FUNG}

\author{
for the Degree of Joint Doctor of Philosophy \\ at The University of Hong Kong and King's College London \\ in August 2017
}

In this thesis, three important topics in actuarial science and financial mathematics are investigated, namely, optimal reinsurance design, risk measures, and forward performance processes.

For the first topic, two general problems of optimal reinsurance design are solved. The first one is the minimization of a general functional of the expectation, Value-at-Risk, and Tail Value-at-Risk of the total retained loss with the convex order preserving premium principle and the budget constraint. Karlin-NovikoffStoyan-Taylor (multiple) crossing conditions are applied to solve the first general problem. The second problem is the minimization of a general law-invariant coherent risk measure of the total retained loss with the law-invariant coherent premium principle and the budget constraint. Representations in terms of distortion functions, application of the mini-max theorem in the infinite dimensional space, and Neyman-Pearson argument are applied to solve the second general problem.

For the second topic, the forward entropic risk measures are investigated. Un- 
der the stochastic factor market model, by making use of the ergodic backward stochastic differential equation representation of the exponential forward investment performance process, a finite horizon backward stochastic differential equation representation of the forward entropic risk measure is obtained. By utilizing the finite horizon backward stochastic differential equation representation of the forward entropic risk measure, the large maturity behavior of the forward entropic risk measure for the risk positions that are deterministic functions of the stochastic factor processes is studied. Specifically, the forward entropic risk measure converges to a constant, which is independent of the initial value of the stochastic factor processes, with an exponential convergence rate. An example with numerical illustrations are demonstrated.

For the third topic, under the stochastic factor market model, an infinite horizon backward stochastic differential equation representation of the exponential forward investment and consumption performance process is obtained. 


\section{ACKNOWLEDGEMENTS}

The author would like to express his deepest gratitude to his supervisors, Dr. Ka Chun Cheung and Dr. Gechun Liang, for their continual guidance and constant encouragement throughout the period of the author's postgraduate studies and in the preparation of this thesis. The author is also very grateful to his collaborators Prof. Ying Hu, Dr. Ambrose Lo, Dr. Sheung Chi Phillip Yam, and Prof. Thaleia Zariphopoulou, for their invaluable comments and insights on the subjects of this thesis.

The author wants to thank Dr. Yingying Lai, Dr. Chi Chung Siu, Dr. Kwok Chuen Wong, Dr. Zheng Zhang, Man Ho Michael Chau, Ki Wai Chau, and Yiqun Li, for their supports and many helpful discussions. The author also wants to thank Tomasz Kosmala, Asuka Kumon, Udomsak Rakwongwan, Kwok Wing Ghaleo Tsoi, Umesh Umesh, Dr. Peter Young, Dr. Menglan April Jiang, Lin Li, Preeya Samani, Shuai James Yuan, Aijin Li, Hui Yan Suzanne Wong, and Yixuan Ellie Zhu, for their cares in the period of the author's postgraduate studies in London.

The author is deeply grateful to his family in Hong Kong, for their precious love, cares, and supports. 


\section{Contents}

\begin{tabular}{lll}
\hline & Introduction & 9
\end{tabular}

1.1 Risk Measures . . . . . . . . . . . . . . . . . . . . . . . . . . . . . 10

1.2 Optimal Reinsurance Design . . . . . . . . . . . . . . . . . . . 13

1.3 Forward Performance Processes . . . . . . . . . . . . . . . . . . . 16

1.4 Main Contributions and Outline . . . . . . . . . . . . . . . . . 19

2 Optimal Reinsurance Design 22

2.1 General Problem Formulation . . . . . . . . . . . . . . . . . . . . 22

2.2 Optimal Reinsurance with Tail Risk Measuring Objective and Convex Order Preserving Premium . . . . . . . . . . . . . . . . . . 24

2.2 .1 Problem Formulation . . . . . . . . . . . . . . . . . . . . 24

2.2 .2 Sufficient Conditions for Convex Ordering . . . . . . . . . 25

2.2 .3 Main Results . . . . . . . . . . . . . . . . . . . . 26

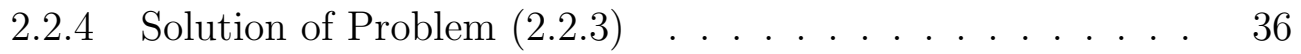

$2.2 .5 \quad$ Solutions of Sub-problems of Problem (2.2.3) . . . . . . . 38

2.3 Optimal Reinsurance with General Coherent Risk Measuring Objective and Premium . . . . . . . . . . . . . . . . . . . 44

2.3 .1 Problem Formulation . . . . . . . . . . . . . . . . . . . . . 44

$2.3 .2 \quad$ First Step: In Terms of Distortion Functions . . . . . . . . 45

2.3.3 Second Step: Interchange of Infimum and Supremum . . . 50

2.3.4 Third Step: Solving Auxiliary Optimal Reinsurance Problem 52 
2.3.5 Second Step Continued: Conditions for Mini-max Theorem 59

$2.3 .6 \quad$ Solution of Problem (2.3.8) . . . . . . . . . . . . 64

3 Forward Entropic Risk Measures $\quad 66$

3.1 Motivation of Forward Entropic Risk Measures: Maturity-independent Risk Measurement . . . . . . . . . . . . . . . . . 66

3.2 Forward Entropic Risk Measures . . . . . . . . . . . . . . . . . . 69

3.3 Stochastic Factor Market Model . . . . . . . . . . . . . . . . . . . 71

$3.4 \quad$ Ergodic BSDE Representation for Exponential Forward Investment Performance Processes . . . . . . . . . . . . . . . . . . . . 73

3.5 BSDE Representation of Forward Entropic Risk Measures . . . . 75

$3.6 \quad$ Large Maturity Behavior of Forward Entropic Risk Measures . . . 84

3.6.1 Markovian Exponential Forward Investment Performance Processes . . . . . . . . . . . . . 86

3.6.2 Non-Markovian Exponential Forward Investment Performance Processes . . . . . . . . . . . . . . . . . . . . 99

3.7 Example and Numerical Illustrations

4 Forward Investment and Consumption Performance Processes 107

4.1 Motivation . . . . . . . . . . . . . . . . . . . . . . . . 107

4.2 Forward Investment and Consumption Performance Processes . . 108

4.3 Stochastic Factor Market Model . . . . . . . . . . . . . . . . . . . 110

4.4 Infinite Horizon BSDE Representation of Exponential Forward Investment and Consumption Performance Processes . . . . . . . . 112

\begin{tabular}{lr}
\hline Bibliography & 118
\end{tabular} 


\section{List of Figures}

$3.1 \quad$ Forward entropic risk measure against the maturity $T$ with $\gamma=1$, $\alpha=0.1, K_{1}=K_{2}=10, \kappa_{1}=0.9, \kappa_{2}=0.1 ;$ blue upward-pointing triangle for $v=5$, red circle for $v=7.5$, green asterisk for $v=10$, black cross for $v=12.5$ and magenta square $v=15$. . . . . . . . . 104

3.2 Forward entropic risk measure against the maturity $T$ with $\gamma=1$, $\alpha=0.1, K_{1}=K_{2}=10, \kappa_{1}=0.5, \kappa_{2}=0.5 ;$ blue upward-pointing triangle for $v=5$, red circle for $v=7.5$, green asterisk for $v=10$, black cross for $v=12.5$ and magenta square $v=15$. . . . . . . . . 105

$3.3 \quad$ Forward entropic risk measure against the maturity $T$ with $\gamma=1$, $\alpha=0.1, K_{1}=K_{2}=10, \kappa_{1}=0.0, \kappa_{2}=1.0 ;$ blue upward-pointing triangle for $v=5$, red circle for $v=7.5$, green asterisk for $v=10$, black cross for $v=12.5$ and magenta square $v=15$. . . . . . . . . 106 


\section{Chapter 1}

\section{Introduction}

Consider the following simple scenario between an insurer and a reinsurer. The insurer, who confronts a future loss for her policyholder, seeks for a risk sharing by purchasing a reinsurance contract from the reinsurer. The reinsurer then price the contract by evaluating the shared risk with the insurer. From the perspective of the insurer, she would like to perform the best risk sharing by signing the optimal reinsurance contract with the reinsurer. On the other hand, from the perspective of the reinsurer, she would like to lay down a universal pricing mechanism to assess any shared risk with the insurer. These two fundamental interests for the insurer and the reinsurer, respectively, motivated the study of the optimal reinsurance design and premium principles, in actuarial science, or risk measures, in finance, which are the first two topics of this thesis, in the literature. We delay the motivation of the third topic of this thesis, namely, the forward performance processes, after we introduce the indifference pricing approach for risk measures.

In the following, we shall give a review of these topics, in the order of, risk measures, optimal reinsurance design, and forward performance processes. 


\section{$1.1 \quad$ Risk Measures}

Risk measures or premium principles are simply functionals which assign every risk position in consideration to a real number. The study of risk measures or premium principles is thus to design these kind of functionals which lead to reasonable risk measurements. We hereby list some desirable properties of risk measures or premium principles which shall appear in this thesis. Denote $X$ and $Y$ as the losses and $\rho$ as the risk measure or premium principle.

1. Translation invariance: for any constant $c \in \mathbb{R}, \rho(X+c)=\rho(X)+c$;

2. Monotonicity: if $X \leq Y$ a.s., then $\rho(X) \leq \rho(Y)$;

3. Convexity: for any constant $\lambda \in[0,1], \rho(\lambda X+(1-\lambda) Y) \leq \lambda \rho(X)+(1-$ $\lambda) \rho(Y)$;

4. Positive homogeneity: for any constant $c \geq 0, \rho(c X)=c \rho(X)$;

5. Comonotonic additivity: if $X$ and $Y$ are comonotonic, then $\mathbb{E}[X+Y]=$ $\mathbb{E}[X]+\mathbb{E}[Y]$

6. Law invariance: $\rho(X)$ depends on $X$ via the distribution of $X$ only;

7. Continuity from above: if $\left\{X_{n}\right\}_{n \in \mathbb{N}}$ is a sequence of risks such that $X_{n} \downarrow X$, then $\rho\left(X_{n}\right) \downarrow \rho(X)$.

In the literature, there are three main approaches of designing the functionals.

The first approach is called the ad-hoc approach. This approach simply assumes that the risk measures or premium principles take particular reasonable forms. After that, the risk measures or premium principles are examined via their properties. Gerber (1979) and Goovaerts et al. (1984) are monographs of 
this approach. In the last decade, this approach was re-adopted to introduce the Value-at-Risk and Tail Value-at-Risk:

$$
\begin{aligned}
\operatorname{VaR}_{\alpha}(X) & \triangleq F_{X}^{-1}(\alpha) \\
& =\inf \left\{x \in[0, \operatorname{ess} \sup (X)] \mid F_{X}(x) \geq \alpha\right\} \\
& =\inf \left\{y \in[0, \operatorname{ess} \sup (Y)] \mid S_{X}(x) \leq 1-\alpha\right\} \\
& =S_{X}^{-1}(1-\alpha)
\end{aligned}
$$

and

$$
\operatorname{TVaR}_{\alpha}(X) \triangleq \begin{cases}\frac{1}{1-\alpha} \int_{\alpha}^{1} \operatorname{VaR}_{\lambda}(X) d \lambda, & \text { if } \alpha \in[0,1), \\ \operatorname{ess} \sup (X), & \text { if } \alpha=1,\end{cases}
$$

where $\alpha \in[0,1]$ is a risk level, $F_{X}$ is the distribution function of $X$, and $S_{X}$ is the survival function of $X$. For comprehensive studies of the properties of Value-atRisk and Tail Value-at-Risk, see, for example, Denuit et al. (2005), Dhaene et al. (2006) and Kaas et al. (2008).

The second approach is the axiomatic approach, which is the converse of the first approach. Instead of assuming the forms of the risk measures or premium principles in the first place, this approach deduces the functionals of the risk measures or premium principles from a set of prescribed reasonable axioms that the risk measures or premium principles should satisfy. For instance, Wang et al. (1997) developed the Wang's premium principle by this axiomatic approach. After that, Artzner et al. (1999) initiated the coherent risk measure theory while Föllmer and Schied (2002) and Frittelli and Gianin (2002) further developed the convex risk measure theory. A risk measure is called coherent risk measure if it satisfies the properties of translation invariance, monotonicity, convexity, and positive homogeneity. A risk measure is called convex risk measure if it satisfies the properties of translation invariance, monotonicity, and convexity. It is wellknown that Value-at-Risk is not a coherent risk measure while Tail Value-at-Risk 
is a coherent risk measure. Föllmer and Schied (2010) is a monograph of this axiomatic approach.

The third approach is the indifference pricing approach. The risk measure or premium principle of a risk position is defined as a solution of an implicit equation by equating two objective values. One of the objective values is the value function of the initial wealth of the seller, while another objective value is the value function of the total wealth of the seller, comprising of her initial wealth, the risk position, and the price of the risk position defined by the risk measure or premium principle. In other words, the risk measure or premium principle of the risk position is a quantity that the seller should receive so that she will be indifferent regardless of the decision made at the beginning. Generally speaking, for the risk position $X$, the value function $V$ of the seller, and the initial wealth $x$ of the seller, the indifference price $\rho(X)$ of the risk position is defined as a solution of the equation:

$$
V(x, 0)=V(x+\rho(X), X) .
$$

If the value functional $V$ is the classical expected utility, with the utility function denoted by $u$, the indifference price $\rho(X)$ defined by

$$
u(x)=\mathbb{E}[u(x+\rho(X)-X)],
$$

is known as the premium principle of zero utility in actuarial science. See, for instance, Bühlmann (1970), Gerber (1979) and Goovaerts et al. (1984). In finance, the value function of the seller includes the investment performance as well. Mathematically, under the classical expected utility paradigm, for the risk position $\xi$ which matures at time $T$, the utility function $u_{T}$ at time $T$, the wealth process $X^{\pi, x}$ with the initial wealth $x$ adapting the investment strategy $\pi$, the indifference price $\rho(\xi)$ of the risk position is defined as a solution of the equation:

$$
\sup _{\pi} \mathbb{E}\left[u_{T}\left(X_{T}^{\pi, x}\right)\right]=\sup _{\pi} \mathbb{E}\left[u_{T}\left(X_{T}^{\pi, x+\rho(\xi)}-\xi\right)\right]
$$


Since Hodges and Neuberger (1989) first proposed this indifference pricing approach to valuate a contingent claim in finance, many works, for instance, in El Karoui and Rouge (2000), Henderson (2002), Henderson and Liang (2014), Hu et al. (2005), Mania and Schweizer (2005), and Musiela and Zariphopoulou (2004), further investigated and enhanced this indifference pricing theory in the last decade. Carmona (2009) is a monograph of these indifference pricing theories. We shall review this indifference pricing approach again when we give the motivation of studying forward performance processes.

The risk measure theory was also extended from the static setting to the dynamic setting by incorporating the availability of the information and the continuous supervision of the risk position. See, for instance, Detlefsen and Scandolo (2005), Jobert and Rogers (2008), Klöppel and Schweizer (2007), and Riedel (2004). Using the $g$-expectation, Coquet et al. (2002) and Peng (2004) provided a linkage between the dynamic risk measures and backward stochastic differential equations (BSDEs).

A remark should be made here that risk measures or premium principles are defined on the random variables in terms of losses in this chapter and Chapter 2 . In Chapter 3, the risk measures are defined on the random variables in terms of gains or payoffs. Hence, some inequalities in Chapter 3 would have different signs comparing to the properties listed above.

\subsection{Optimal Reinsurance Design}

The problem of the optimal (re)insurance design aims to find the optimal (re)insurance indemnity function such that the agent optimizes her objective. These were originated from the founded works by Borch (1960), Ohlin (1969), Arrow (1974), and Raviv (1979). Borch (1960) minimized the variance of the retained loss; Ohlin 
(1969) minimized a measure of the dispersion of the retained and ceded loss; Arrow (1974) maximized the expected utility of the terminal wealth; Raviv (1979) solved the Pareto optimality when both the policyholder and the insurer maximizing the expected utility.

In the last two decades, the problems of the optimal (re)insurance design have been caught attention again. To name a few, Gollier and Schlesinger (1996) extended the result of Arrow (1974) by maximizing an increasing convex order preserving objective of the terminal wealth; Young (1999) extended the result of Arrow (1974) by considering the Wang's premium principle; Kaluszka (2001) extended the result of Borch (1960) by considering the mean-variance premium principle; Kaluszka (2004) minimized a general expected convex functional of the retained loss with a general increasing concave premium principle; Kaluszka (2005) continued the study in Kaluszka (2004) with a general convex premium principle; Promislow and Young (2005) provided a universal argument for the solutions of the problems appeared so far at that time; Cai and Tan (2007) solved the optimal retention level of a stop-loss reinsurance by minimizing the Value-at-Risk and Conditional Tail Expectation of the retained loss; Cai et al. (2008) extended the result of Cai and Tan (2007) by solving general optimal reinsurance indemnity with the same objective functions, namely, Value-at-Risk and Conditional Tail Expectation of the retained loss; Kaluszka and Okolewski (2008) extended the result of Arrow (1974) with the convex combination of the expected value and the maximal loss premium principle; Balbás et al. (2009) extended the result in Cai and Tan (2007) by minimizing a general risk measure of the retained loss; Tan et al. (2009) extended the result of Cai and Tan (2007) by solving optimal quota-share as well; Cheung (2010) revisited the problem of Cai et al. (2008) and extended their result with the Wang's premium principle; Sung et al. (2011) maximized the objective using the Cumulative Prospect Theory by Kahneman and Tversky (1992); Tan et al. (2011) extended the result in Cai et al. (2008) with premium constraints; Cai 
and Wei (2012) solved the multivariate optimal reinsurance design problem with positively dependent risks; Chi (2012a) extended the result of Cai et al. (2008) with variance related premium principles; Chi (2012b) minimized the Value-atRisk and Tail Value-at-Risk of the risk-adjusted liability with the convex order preserving premium principle; Asimit et al. (2013) minimized the Value-at-Risk and general distortion risk measure of the retained loss under the presence of the default risk by the reinsurer; Cheung et al. (2013) solved the multivariate optimal reinsurance design problem with a general law-invariant convex risk measure and the worst case dependence structure; Chi and Tan (2013) extended the result in Cai et al. (2008) with a general stop-loss order preserving premium principle; Chi and Weng (2013) extended the result in Chi (2012b) by restricting the feasible indemnities which satisfy the Vajda condition; Cui et al. (2013) minimized a general distortion risk measure of the retained loss with the distortion premium principle; Cheung et al. (2014) minimized a general law-invariant convex risk measure of the retained loss; Zheng and Cui (2014) extended the result in Cui et al. (2013) with the premium constraint; Cheung et al. (2015b) maximized the disappointment utilities by various disappointment models of the terminal wealth with the convex combination of the expected value and the maximal loss premium principle; Bernard et al. (2015) maximized the rank-dependent utility of the terminal wealth; Cheung and Lo (2017) used a cost-benefit argument to extend the result in Cui et al. (2013); Boonen et al. (2016) investigated the effect on the optimal solutions in the presence of the representative reinsurer; Cai et al. (2016) minimized the convex combination of the Value-at-Risk of the loss of the insurer and the reinsurer, together with two different constraints; Cai and Weng (2016) extended the result in Chi (2012b) with the risk margin in the liability measured by the expectile; Zhuang et al. (2016) solved some three party problems of the optimal insurance and reinsurance designs; Lo (2017) employed the Neyman-Pearson type argument to solve three general classes of optimal reinsurance problems. 


\subsection{Forward Performance Processes}

To motivate the forward performance processes, we recall the indifference pricing approach in risk measures. Under the classical expected utility framework, for the risk position $\xi$ which matures at time $T$, the utility function $u_{T}$ at time $T$, the wealth process $X^{\pi, t, x}$ with the initial wealth $x$ at the initial time $t \in[0, T]$ adapting the investment strategy $\pi$, the indifference price $\rho_{t}(\xi)$ of the risk position $\xi$ at time $t$ is defined by

$$
\sup _{\pi} \mathbb{E}\left[u_{T}\left(X_{T}^{\pi, t, x}\right) \mid \mathcal{F}_{t}\right]=\sup _{\pi} \mathbb{E}\left[u_{T}\left(X_{T}^{\pi, t, x+\rho_{t}(\xi)}-\xi\right) \mid \mathcal{F}_{t}\right] .
$$

Hence, to determine the indifference price $\rho_{t}(\xi)$ is essentially to solve two classical Merton's type stochastic optimization problems.

In particular, the left hand side of the equation describes the performance of the investment through the expected terminal utility criteria, given the initial wealth $x$ at the initial time $t \in[0, T]$. Define the implied value function

$$
u(x, t ; T)=\sup _{\pi} \mathbb{E}\left[u_{T}\left(X_{T}^{\pi, t, x}\right) \mid \mathcal{F}_{t}\right], \quad \forall t \in[0, T],
$$

which can be intuitively regarded as a dynamic utility of the investor. Moreover, the value function $u(\cdot, \cdot ; T)$ satisfies the dynamic programming principle that

$$
u(x, t ; T)=\sup _{\pi} \mathbb{E}\left[u\left(X_{s}^{\pi, t, x}, s ; T\right) \mid \mathcal{F}_{t}\right], \quad \forall t \in[0, T], s \in[t, T] .
$$

This entails that, for any investment strategy $\pi$,

$$
u(x, t ; T) \geq \mathbb{E}\left[u\left(X_{s}^{\pi, t, x}, s ; T\right) \mid \mathcal{F}_{t}\right], \quad \forall t \in[0, T], s \in[t, T],
$$

while, if the supremum can be achieved, there exists an investment strategy $\pi^{*}$ such that

$$
u(x, t ; T)=\mathbb{E}\left[u\left(X_{s}^{\pi^{*}, t, x}, s ; T\right) \mid \mathcal{F}_{t}\right], \quad \forall t \in[0, T], s \in[t, T] .
$$


This further implies that $u\left(X_{t}^{\pi, x}, t ; T\right)$ is a supermartingale for any investment strategy $\pi$ while $u\left(X_{t}^{\pi^{*}, x}, t ; T\right)$ is a martingale for some optimal investment strategy $\pi^{*}$ on the investment horizon $[0, T]$.

Note that the above backward assessment of the investment performance could be achieved only if the duration $T$ of the investment horizon is fixed and the utility $u_{T}$ at time $T$ is exogenously chosen at the very beginning. Due to these two implicit assumptions, the indifference price $\rho_{t}$ cannot evaluate any risk positions matured beyond the prescribed maturity time $T$; the implied value function $u(\cdot, \cdot ; T)$ is generated backwardly, an opposite direction comparing to the forward evolution of the market. Most importantly, the second assumption enforces the investor to determine the current investment decisions using the assumed utility in the future, which is definitely not natural in practice.

To remedy the above issues arising from the classical expected utility maximization problem, Musiela and Zariphopoulou (2005, 2007, 2008, 2009a, b, 2010a,b) introduced and developed the notion of forward performance processes. Roughly speaking, instead of assuming a utility function at a future time point, the utility function $u_{0}$ is chosen at the initial time which is naturally known, or at least can be estimated, at the very beginning. Given the assumed initial utility function $u_{0}$, an adapted implied value process $U(\cdot, \cdot)$, with

$$
U(x, 0)=u_{0}(x)
$$

is generated forwardly by the criteria motivated from the classical expected utility paradigm:

(i) for any investment strategy $\pi$,

$$
U(x, t) \geq \mathbb{E}\left[U\left(X_{s}^{\pi, t, x}, s\right) \mid \mathcal{F}_{t}\right], \quad \forall t \geq 0, s \geq t
$$

(ii) there exists an investment strategy $\pi^{*}$ such that

$$
U(x, t)=\mathbb{E}\left[U\left(X_{s}^{\pi^{*}, t, x}, s\right) \mid \mathcal{F}_{t}\right], \quad \forall t \geq 0, s \geq t
$$


This implied value process is called the forward performance process. One should realize that the performance process $U(\cdot, \cdot)$ can be defined forwardly at all times $t \geq 0$, comparing to that the performance process $u(\cdot, \cdot ; T)$, under the classical expected utility framework, can only be defined backwardly on the fixed investment horizon $[0, T]$.

The above criteria only includes the investment performance, and thus the above process $U(\cdot, \cdot)$ is more precisely called the forward investment performance process. Naturally, Berrier and Tehranchi (2008) and Källblad (2016) extended the forward investment performance processes, by implementing the consumption component $C$ into the supermartingale and martingale optimality criteria, to introduce the adapted forward investment and consumption performance processes $U(\cdot, \cdot)$ and $U^{c}(\cdot, \cdot)$. Mathematically, given the assumed initial utility functions $u_{0}$ and $u_{0}^{c}$, a pair of adapted forward investment and consumption performance processes $U(\cdot, \cdot)$ and $U^{c}(\cdot, \cdot)$, with

$$
U(x, 0)=u_{0}(x)
$$

and

$$
U^{c}(C, 0)=u_{0}^{c}(C)
$$

is generated forwardly by the following criteria:

(i) for any investment strategy $\pi$ and consumption rate $C$,

$$
U(x, t) \geq \mathbb{E}\left[U\left(X_{s}^{\pi, C, t, x}, s\right)+\int_{t}^{s} U^{c}\left(C_{u}, u\right) d u \mid \mathcal{F}_{t}\right], \quad \forall t \geq 0, s \geq t
$$

(ii) there exist investment strategy $\pi^{*}$ and consumption rate $C^{*}$ such that

$$
U(x, t)=\mathbb{E}\left[U\left(X_{s}^{\pi^{*}, C^{*}, t, x}, s\right)+\int_{t}^{s} U^{c}\left(C_{u}^{*}, u\right) d u \mid \mathcal{F}_{t}\right], \quad \forall t \geq 0, s \geq t .
$$

The forward investment performance processes and the forward investment and consumption performance processes will be respectively defined more properly in Chapter 3 and Chapter 4 after the market models are introduced. 


\subsection{Main Contributions and Outline}

In the first part of this thesis, we solve two general optimal reinsurance problems in Chapter 2. The first one is the minimization of a general functional of the expectation, Value-at-Risk, and Tail Value-at-Risk of the total retained loss of the insurer while the premium principle determined by the reinsurer is a convex order preserving functional, together with the budget constraint. To solve this first general problem, instead of using the classical Karlin-Novikoff once-crossing criterion as in the literature, we apply a generalization of it, namely, the Karlin-NovikoffStoyan-Taylor (multiple) crossing conditions. To the best of our knowledge, this is the first application of the multiple crossing conditions to solve optimal reinsurance problems. Our results in the first problem are generalization of those in Chi (2012b). The second problem is the minimization of a general law-invariant continuous from above coherent risk measure of the total retained loss of the insurer while the premium principle determined by the reinsurer is a law-invariant comonotonic additive convex risk measure, together with the budget constraint. To solve this second general problem, we first represent the objective function and the premium principle in terms of distortion functions; we then apply the minimax theorem for infinite dimensional space to interchange the infimum on the space of indemnities and the supremum on the space of probability measures; we finally employ the recent results by Lo (2017) to solve the minimization problem. Our results in the second problem can be regarded as a partial generalization of Cheung et al. (2014), in which they minimized a general law-invariant convex risk measure but with the expected value premium principle and without the budget constraint.

In Chapter 3, the second part of this thesis, we study the forward entropic risk measures, first introduced by Zariphopoulou and Žitković (2010). The forward entropic risk measures, similar to the classical entropic risk measures, are 
defined in a decision theoretic framework using the indifference pricing approach. Moreover, the forward entropic risk measures are maturity independent risk measures, which were first introduced in Zariphopoulou and Žitković (2010) to remedy the deficiency in the classical dynamic risk measures. These motivated us to further investigate the behavior of the forward entropic risk measures. The important ingredient in the forward entropic risk measure is the exponential forward investment performance process. By making use of the ergodic BSDE representation of the exponential forward investment performance process by Liang and Zariphopoulou (2017), we provide a finite horizon BSDE representation of the forward entropic risk measure under a stochastic factor market model. By utilizing the BSDE representation of the risk measure, we study the large maturity behavior of the risk measure for any risk positions Markovian with respect to the stochastic factor. In particular, we showed that the forward entropic risk measure converges to a constant, which is independent of the initial value of the stochastic factor, with an exponential convergence rate. We also give a simple example together with numerical illustrations to echo the theoretical convergence result.

The third part of this thesis is motivated by the works in the second part. In particular, a crucial element in the second part of this thesis is the ergodic BSDE representation of the exponential forward investment performance process by Liang and Zariphopoulou (2017). Without that representation, the forward entropic risk measures are by no means represented by finite horizon BSDEs, and hence the study of their large maturity behaviors. Therefore, in Chapter 4 , the third part of this thesis, we show an infinite horizon BSDE representation of the exponential forward investment and consumption performance process under the stochastic factor market model. This representation result would probably be applied in future works.

The works in this thesis are based on the joint works in Cheung et al. (2015b), 
Cheung et al. (2017), Chong et al. (2017), and Chong and Liang (2017). 


\section{Chapter 2}

\section{Optimal Reinsurance Design}

\subsection{General Problem Formulation}

Let $X$ be a non-negative integrable random variable defined on a probability space $(\Omega, \mathcal{F}, \mathbb{P})$ to model an insurable loss of an insurer over a fixed period of time. Denote $F_{X}, S_{X}$, and $\operatorname{supp}(X)$ as the distribution function, the survival function, and the support of the random variable $X$, respectively. Assume that $0 \in \operatorname{supp}(X)$.

To reduce the risk exposure, the insurer seeks for a reinsurance protection from a reinsurer, who is responsible for a part of the loss, which is called the ceded loss and denoted by $I(X)$, where the ceded loss function $I$ satisfies certain reasonable assumptions to be specified below, and the insurer pays a non-negative premium $P(I(X))$ which is determined by the reinsurer. Consequently, the insurer retains the remaining part of the loss $X-I(X)$, which is called the retained loss; and hence, together with the premium paid, the total retained loss of the insurer is

$$
T_{I}(X) \triangleq X-I(X)+P(I(X)) .
$$

Any reasonable ceded loss payment by the reinsurer should satisfy the following intrinsic properties: 
(i) if the insurer does not report any loss, the payment is zero;

(ii) the payment is non-negative and is at most the loss reported by the insurer;

(iii) to avoid moral hazard, a unit increment of the payment is less than a unit increment of the loss reported by the insurer.

Mathematically, any feasible ceded loss function $I$ should satisfy:

(i) $I(0)=0$;

(ii) $0 \leq I(x) \leq x$, for any $x \in \operatorname{supp}(X)$;

(iii) $0 \leq I(x)-I(y) \leq x-y$, for any $x, y \in \operatorname{supp}(X)$ with $y<x$.

The condition (iii) is usually referred as 1-Lipschitz condition. With $y=0$, conditions (i) and (iii) implies the condition (ii). In the sequel, we assume that any feasible ceded loss function $I$ lies in the set $\mathcal{I}$ which is defined by

$$
\begin{aligned}
\mathcal{I} \triangleq\{I: \operatorname{supp}(X) \rightarrow[0, \operatorname{ess} \sup (X)]: & \\
& I(0)=0 \text { and } 0 \leq I(x)-I(y) \leq x-y, \forall x, y \in \operatorname{supp}(X) \text { with } y<x\} .
\end{aligned}
$$

By the condition (iii), for any $I \in \mathcal{I}$, both $I$ and $\mathrm{Id}-I$ are non-decreasing functions. Since the functions $I \in \mathcal{I}$ are Lipschitz continuous, they are absolutely continuous as well.

The optimal reinsurance design problem for the insurer is to search for the best ceded loss function to purchase from the reinsurer, by balancing the benefit of the payment from the reinsurer and the cost of the premium to the reinsurer, and meeting the budget constraint. Mathematically, the insurer solves the following constrained optimization problem:

$$
\begin{cases}\inf _{I \in \mathcal{I}} & V\left(T_{I}(X)\right), \\ \text { s.t. } & P(I(X)) \leq \pi,\end{cases}
$$


for some appropriate objective function $V$, a premium principle $P$, and a budget $\pi \in \mathbb{R}_{+}$. Two of the optimization problems (2.1.2) shall be studied in the following two respective sections.

In the sequel, we assume that the probability space $(\Omega, \mathcal{F}, \mathbb{P})$ is atomless, so that it supports a continuous distribution.

\subsection{Optimal Reinsurance with Tail Risk Mea- suring Objective and Convex Order Preserv- ing Premium}

\subsubsection{Problem Formulation}

In this section, we consider the scenario that the insurer minimizes a general function of the expectation, Value-at-Risk, and Tail Value-at-Risk of her total retained loss while the premium principle determined by the reinsurer preserves convex ordering. Mathematically, the insurer solves:

$$
\begin{cases}\inf _{I \in \mathcal{I}} & G\left(\mathbb{E}\left[T_{I}(X)\right], \operatorname{VaR}_{\alpha}\left(T_{I}(X)\right), \operatorname{TVaR}_{\alpha}\left(T_{I}(X)\right)\right), \\ \text { s.t. } & g\left(\mathbb{E}[I(X)], v_{\mathrm{cx}}(I(X))\right) \leq \pi\end{cases}
$$

where $G$ is a real-valued function on $\mathbb{R}_{+}^{3}$, non-decreasing in all arguments, $g$ is a non-negative real-valued function on $\mathbb{R}_{+}^{2}$, non-decreasing in both arguments, $v_{\mathrm{cx}}$ is a convex order preserving functional, in the sense that if $Y \leq_{\mathrm{cx}} Z$ then $v_{\text {cx }}(Y) \leq v_{\text {cx }}(Z), \alpha \in(0,1)$ is a threshold risk level, and $\pi \in \mathbb{R}_{+}$is the budget. Recall that $Y \leq_{\mathrm{cx}} Z$ if $\mathbb{E}[f(Y)] \leq \mathbb{E}[f(Z)]$ for any convex function $f$. The premium principle in Problem 2.2.3) is very general that it includes many classical premium principles, namely, expected value, Wang's, Dutch, variance, and standard deviation principles. 
To avoid unnecessary technical details, assume that the loss $X$ is continuous with a strictly increasing distribution function $F_{X}$. Our analysis and results can be easily generalized to any loss with a mixed distribution, which, in particular, includes the case that the loss $X$ has a point mass at 0 , which is ordinary in practice.

\subsubsection{Sufficient Conditions for Convex Ordering}

Since Problem (2.2.3) involves a convex order preserving functional, it is natural to apply the convex ordering approach on solving it. The approach makes use of sufficient conditions for convex orderings by the crossing(s) of the distribution functions. Before stating the conditions, we first define comprehensibly the meaning of "crossing".

Definition 2.2.1. Let $f_{1}$ and $f_{2}$ be two real-valued functions on $[a, b]$ with $a<b$. These two functions $f_{1}$ and $f_{2}$ are said to be crossing $n \in \mathbb{N}$ times if these exist ordered points in $(a, b)$ :

$$
\xi_{1}^{L} \leq \xi_{1}^{R}<\xi_{2}^{L} \leq \xi_{2}^{R}<\cdots<\xi_{n}^{L} \leq \xi_{n}^{R},
$$

such that, for each $i=1,2, \ldots, n$,

(i) for any $x \in\left(\xi_{i-1}^{R}, \xi_{i}^{L}\right)$ and $y \in\left(\xi_{i}^{R}, \xi_{i+1}^{L}\right)$,

$$
\left(f_{1}(x)-f_{2}(x)\right)\left(f_{1}(y)-f_{2}(y)\right)<0,
$$

where $\xi_{0}^{R}=a$ and $\xi_{n+1}^{L}=b$;

(ii) if $\xi_{i}^{L}<\xi_{i}^{R}$, for any $x \in\left(\xi_{i}^{L}, \xi_{i}^{R}\right), f_{1}(x)=f_{2}(x)$.

To facilitate the use of convex orderings, Karlin and Novikoff (1963) provided sufficient conditions in terms of distribution functions, known as Karlin-Novikoff once-crossing criterion. 
Theorem 2.2.2 (Karlin-Novikoff once-crossing criterion). Let $Y$ and $Z$ be two random variables with respective distribution functions $F_{Y}$ and $F_{Z}$. Assume that all of the following conditions are satisfied:

(i) $\mathbb{E}[Y]=\mathbb{E}[Z]$;

(ii) $F_{Y}$ and $F_{Z}$ cross once;

(iii) for any $x \in\left(a, \xi_{1}^{L}\right), F_{Y}(x)<F_{Z}(x)$, where $a=\inf \left\{x: F_{Y}(x) \neq F_{Z}(x)\right\}$.

Then $Y \leq_{c x} Z$

However, to solve Problem (2.2.3), the following generalization, by Stoyan (1983) and Taylor (1983), known as Karlin-Novikoff-Stoyan-Taylor crossing conditions, will be more handy.

Theorem 2.2.3 (Karlin-Novikoff-Stoyan-Taylor crossing conditions). Let $Y$ and $Z$ be two random variables with respective distribution functions $F_{Y}$ and $F_{Z}$. Assume that $F_{Y}$ and $F_{Z}$ cross $n \in \mathbb{N}$ times. Then $Y \leq_{c x} Z$ if and only if all of the following conditions are satisfied:

(i) $\mathbb{E}[Y]=\mathbb{E}[Z]$;

(ii) for any $x \in\left(a, \xi_{1}^{L}\right), F_{Y}(x)<F_{Z}(x)$, where $a=\inf \left\{x: F_{Y}(x) \neq F_{Z}(x)\right\}$.

(iii) $n=2 m-1$ for some $m \in \mathbb{N}$, and if $m \neq 1$, for each $j=1,2, \ldots, m-1$, $\pi_{Y}\left(\xi_{2 j}^{R}\right) \leq \pi_{Z}\left(\xi_{2 j}^{R}\right)$, where $\pi_{Y}(\cdot) \triangleq \mathbb{E}\left[(Y-\cdot)_{+}\right]$is the stop-loss transform of $Y$ and $\pi_{Z}(\cdot)$ is the stop-loss transform of $Z$.

\subsubsection{Main Results}

Define the following subsets of $\mathcal{I}$ :

$$
\begin{aligned}
& \mathcal{I}_{3} \triangleq\{I \in \mathcal{I}: \exists d_{1} \in[0, \operatorname{ess} \sup (X)], d_{2} \in\left[d_{1}, \operatorname{ess} \sup (X)\right], d_{3} \in\left[d_{2}, \operatorname{ess} \sup (X)\right]: \\
&\left.\forall x \in[0, \operatorname{ess} \sup (X)], I(x)=x-\left(x-d_{1}\right)_{+}+\left(x-d_{2}\right)_{+}-\left(x-d_{3}\right)_{+}\right\}
\end{aligned}
$$




$$
\begin{aligned}
& \mathcal{I}^{L} \triangleq\left\{I \in \mathcal{I} \backslash \mathcal{I}_{3}: \exists \tau \in[0, \operatorname{ess} \sup (X)], d_{1} \in[0, \tau], d_{2} \in\left[d_{1}, \tau\right]:\right. \\
& \left.\forall x \in[0, \tau], I(x)=x-\left(x-d_{1}\right)_{+}+\left(x-d_{2}\right)_{+}\right\} ; \\
& \mathcal{I}^{R} \triangleq\left\{I \in \mathcal{I} \backslash \mathcal{I}_{3}: \exists \tau \in[0, \operatorname{ess} \sup (X)], d_{3} \in[\tau, \operatorname{ess} \sup (X)]:\right. \\
& \left.\forall x \in[\tau, \operatorname{ess} \sup (X)], I(x)-I(\tau)=x-\left(x-d_{3}\right)_{+}\right\} .
\end{aligned}
$$

Before solving Problem (2.2.3), we prove two theorems, which are main results of this section.

Theorem 2.2.4. For any $I \in \mathcal{I} \backslash \mathcal{I}_{3}$, there exists an $\tilde{I} \in \mathcal{I}_{3}$, with parameters $\tilde{d}_{1} \in[0, \operatorname{ess} \sup (X)], \tilde{d}_{2} \in\left[\tilde{d}_{1}, \operatorname{ess} \sup (X)\right], \tilde{d}_{3} \in\left[\tilde{d}_{2}, \operatorname{ess} \sup (X)\right]$, such that

(i) $\operatorname{VaR}_{\alpha}(X) \in\left[\tilde{d}_{2}, \tilde{d}_{3}\right]$;

(ii) $\mathbb{E}[\tilde{I}(X)]=\mathbb{E}[I(X)]$;

(iii) $\operatorname{VaR}_{\alpha}(\tilde{I}(X))=\operatorname{VaR}_{\alpha}(I(X))$;

(iv) $T \operatorname{VaR} R_{\alpha}(\tilde{I}(X))=T \operatorname{VaR}_{\alpha}(I(X))$;

(v) $\tilde{I}(X) \leq_{c x} I(X)$.

Proof. Let $I \in \mathcal{I} \backslash \mathcal{I}_{3}$. By Intermediate Value Theorem, there exist $\tilde{d}_{1} \in\left[0, \operatorname{VaR}_{\alpha}(X)\right], \tilde{d}_{2} \in$ $\left[\tilde{d}_{1}, \operatorname{VaR}_{\alpha}(X)\right], \tilde{d}_{3} \in\left[\operatorname{VaR}_{\alpha}(X), \operatorname{ess} \sup (X)\right]$, such that

(a) $\mathbb{E}\left[X-\left(X-\tilde{d}_{1}\right)_{+}+\left(X-\tilde{d}_{2}\right)_{+}-\left(X-\tilde{d}_{3}\right)_{+}\right]=\mathbb{E}[I(X)]$

(b) $\operatorname{VaR}_{\alpha}(X)-\tilde{d}_{2}+\tilde{d}_{1}=I\left(\operatorname{VaR}_{\alpha}(X)\right)$;

(c) $\mathbb{E}\left[\left(\left(X-\left(X-\tilde{d}_{1}\right)_{+}+\left(X-\tilde{d}_{2}\right)_{+}-\left(X-\tilde{d}_{3}\right)_{+}\right)-\left(\operatorname{VaR}_{\alpha}(X)-\tilde{d}_{2}+\tilde{d}_{1}\right)\right)_{+}\right]=$ $\mathbb{E}\left[\left(I(X)-I\left(\operatorname{VaR}_{\alpha}(X)\right)\right)_{+}\right]$.

Define

$$
\tilde{I}(x)=x-\left(x-\tilde{d}_{1}\right)_{+}+\left(x-\tilde{d}_{2}\right)_{+}-\left(x-\tilde{d}_{3}\right)_{+}, \quad \forall x \in[0, \operatorname{ess} \sup (X)]
$$


Then, $\tilde{I} \in \mathcal{I}_{3} ;(a)$ is equivalent to that

$$
\mathbb{E}[\tilde{I}(X)]=\mathbb{E}[I(X)] ;
$$

Since both $I$ and $\tilde{I}$ are non-decreasing, by (b),

$$
\begin{aligned}
\operatorname{VaR}_{\alpha}(\tilde{I}(X)) & =\tilde{I}\left(\operatorname{VaR}_{\alpha}(X)\right) \\
& =\operatorname{VaR}_{\alpha}(X)-\tilde{d}_{2}+\tilde{d}_{1} \\
& =I\left(\operatorname{VaR}_{\alpha}(X)\right) \\
& =\operatorname{VaR}_{\alpha}(I(X)) ;
\end{aligned}
$$

Since $\operatorname{VaR}_{\alpha}(\tilde{I}(X))=\operatorname{VaR}_{\alpha}(I(X))$, by $(\mathrm{c})$,

$$
\begin{aligned}
\operatorname{TVaR}_{\alpha}(\tilde{I}(X))= & \operatorname{VaR}_{\alpha}(\tilde{I}(X))+\frac{1}{1-\alpha} \pi_{\tilde{I}(X)}\left(\operatorname{VaR}_{\alpha}(\tilde{I}(X))\right) \\
= & \operatorname{VaR}_{\alpha}(\tilde{I}(X))+\frac{1}{1-\alpha} \mathbb{E}\left[\left(\tilde{I}(X)-\operatorname{VaR}_{\alpha}(\tilde{I}(X))\right)_{+}\right] \\
= & \operatorname{VaR}_{\alpha}(\tilde{I}(X)) \\
& \quad+\frac{1}{1-\alpha} \mathbb{E}\left[\left(\left(X-\left(X-\tilde{d}_{1}\right)_{+}+\left(X-\tilde{d}_{2}\right)_{+}-\left(X-\tilde{d}_{3}\right)_{+}\right)\right.\right. \\
& \left.\left.\quad-\left(\operatorname{VaR}_{\alpha}(X)-\tilde{d}_{2}+\tilde{d}_{1}\right)\right)_{+}\right] \\
= & \operatorname{VaR}_{\alpha}(I(X))+\frac{1}{1-\alpha} \mathbb{E}\left[\left(I(X)-I\left(\operatorname{VaR}_{\alpha}(X)\right)\right)_{+}\right] \\
= & \operatorname{VaR}_{\alpha}(I(X))+\frac{1}{1-\alpha} \mathbb{E}\left[\left(I(X)-\operatorname{VaR}_{\alpha}(I(X))\right)_{+}\right] \\
= & \operatorname{VaR}_{\alpha}(I(X))+\frac{1}{1-\alpha} \pi_{I(X)}\left(\operatorname{VaR}_{\alpha}(I(X))\right) \\
= & \operatorname{TVaR}_{\alpha}(I(X)) ;
\end{aligned}
$$

The distribution function of $\tilde{I}(X)$ is given by

$$
F_{\tilde{I}(X)}(y)= \begin{cases}F_{X}(y), & \forall y \in\left[0, \tilde{d}_{1}\right), \\ F_{X}\left(y+\tilde{d}_{2}-\tilde{d}_{1}\right), & \forall y \in\left[\tilde{d}_{1}, \tilde{d}_{3}-\tilde{d}_{2}+\tilde{d}_{1}\right), \\ 1, & \forall y \in\left[\tilde{d}_{3}-\tilde{d}_{2}+\tilde{d}_{1}, \operatorname{ess} \sup (X)\right] .\end{cases}
$$


To apply the crossing conditions in Theorem 2.2.3, the form of $I \in \mathcal{I} \backslash \mathcal{I}_{3}$ and the value of $\operatorname{VaR}_{\alpha}(X)$ need to be specified more explicitly. Hence, the following different cases are studied. Note that $\mathbb{E}[\tilde{I}(X)]=\mathbb{E}[I(X)]$ has already been shown.

If $I \in \mathcal{I}^{L} \backslash \mathcal{I}^{R}$, with parameters $\tau \in[0, \operatorname{ess} \sup (X)], d_{1} \in[0, \tau], d_{2} \in\left[d_{1}, \tau\right]$, and if $\tau<\operatorname{VaR}_{\alpha}(X)$, the distribution functions $F_{\tilde{I}(X)}$ and $F_{I(X)}$ cross three times with $\xi_{1}^{L}=\xi_{1}^{R}=\tilde{d}_{1}, \xi_{2}^{L}=\xi_{2}^{R}=\operatorname{VaR}_{\alpha}(X)-\tilde{d}_{2}+\tilde{d}_{1}$, and $\xi_{3}^{L}=\xi_{3}^{R}=\tilde{d}_{3}-\tilde{d}_{2}+\tilde{d}_{1}$. For any $y \in\left(d_{1}, \xi_{1}^{L}\right), F_{\tilde{I}(X)}(y)<F_{I(X)}(y)$. Also, by $(c), \pi_{\tilde{I}(X)}\left(\xi_{2}^{R}\right)=\pi_{I(X)}\left(\xi_{2}^{R}\right)$. Hence, by Theorem 2.2.3, $\tilde{I}(X) \leq_{\mathrm{cx}} I(X)$.

If $I \in \mathcal{I}^{L} \backslash \mathcal{I}^{R}$, with parameters $\tau \in[0$, ess $\sup (X)], d_{1} \in[0, \tau], d_{2} \in\left[d_{1}, \tau\right]$, and if $d_{2}<\operatorname{VaR}_{\alpha}(X) \leq \tau$, then $\tilde{d}_{1}=d_{1}$ and $\tilde{d}_{2}=d_{2}$. In this case, the distribution functions $F_{\tilde{I}(X)}$ and $F_{I(X)}$ cross once with $\xi_{1}^{L}=\xi_{1}^{R}=\tilde{d}_{3}-d_{2}+d_{1}$. For any $y \in\left(I(\tau), \xi_{1}^{L}\right), F_{\tilde{I}(X)}(y)<F_{I(X)}(y)$. Hence, by Theorem 2.2.3, $\tilde{I}(X) \leq_{\mathrm{cx}} I(X)$.

If $I \in \mathcal{I}^{L} \backslash \mathcal{I}^{R}$, with parameters $\tau \in[0, \operatorname{ess} \sup (X)], d_{1} \in[0, \tau], d_{2} \in\left[d_{1}, \tau\right]$, and if $d_{1}<\operatorname{VaR}_{\alpha}(X) \leq d_{2}$, then $\tilde{d}_{1}=d_{1}$ and $\tilde{d}_{2}=\operatorname{VaR}_{\alpha}(X)$. In this case, the distribution functions $F_{\tilde{I}(X)}$ and $F_{I(X)}$ cross once with $\xi_{1}^{L}=\xi_{1}^{R}=\tilde{d}_{3}-\operatorname{VaR}_{\alpha}(X)+$ $d_{1}$. For any $y \in\left(d_{1}, \xi_{1}^{L}\right), F_{\tilde{I}(X)}(y)<F_{I(X)}(y)$. Hence, by Theorem 2.2.3. $\tilde{I}(X) \leq_{\mathrm{cx}}$ $I(X)$.

If $I \in \mathcal{I}^{L} \backslash \mathcal{I}^{R}$, with parameters $\tau \in[0, \operatorname{ess} \sup (X)], d_{1} \in[0, \tau], d_{2} \in\left[d_{1}, \tau\right]$, and if $0<\operatorname{VaR}_{\alpha}(X) \leq d_{1}$, then $\tilde{d}_{1}=\tilde{d}_{2}=\operatorname{VaR}_{\alpha}(X)$. In this case, the distribution functions $F_{\tilde{I}(X)}$ and $F_{I(X)}$ cross once with $\xi_{1}^{L}=\xi_{1}^{R}=\tilde{d}_{3}$. For any $y \in\left(d_{1}, \xi_{1}^{L}\right)$, $F_{\tilde{I}(X)}(y)<F_{I(X)}(y)$. Hence, by Theorem 2.2.3. $\tilde{I}(X) \leq_{\mathrm{cx}} I(X)$.

If $I \in \mathcal{I}^{R} \backslash \mathcal{I}^{L}$, with parameters $\tau \in[0$, ess $\sup (X)], d_{3} \in[\tau, \operatorname{ess} \sup (X)]$, and if $d_{3} \leq \operatorname{VaR}_{\alpha}(X)$, then $\tilde{d}_{3}=\operatorname{VaR}_{\alpha}(X)$. In this case, the distribution functions $F_{\tilde{I}(X)}$ and $F_{I(X)}$ cross once with $\xi_{1}^{L}=\xi_{1}^{R}=\tilde{d}_{1}$. For any $y \in\left(0, \xi_{1}^{L}\right), F_{\tilde{I}(X)}(y)<F_{I(X)}(y)$. Hence, by Theorem 2.2.3, $\tilde{I}(X) \leq_{\mathrm{cx}} I(X)$.

If $I \in \mathcal{I}^{R} \backslash \mathcal{I}^{L}$, with parameters $\tau \in[0$, ess $\sup (X)], d_{3} \in[\tau, \operatorname{ess} \sup (X)]$, and if 
$\tau \leq \operatorname{VaR}_{\alpha}(X)<d_{3}$, then $\tilde{d}_{3}=d_{3}$. In this case, the distribution functions $F_{\tilde{I}(X)}$ and $F_{I(X)}$ cross once with $\xi_{1}^{L}=\xi_{1}^{R}=\tilde{d}_{1}$. For any $y \in\left(0, \xi_{1}^{L}\right), F_{\tilde{I}(X)}(y)<F_{I(X)}(y)$. Hence, by Theorem 2.2.3, $\tilde{I}(X) \leq_{\mathrm{cx}} I(X)$.

If $I \in \mathcal{I}^{R} \backslash \mathcal{I}^{L}$, with parameters $\tau \in[0$, ess $\sup (X)], d_{3} \in[\tau, \operatorname{ess} \sup (X)]$, and if $0<\operatorname{VaR}_{\alpha}(X)<\tau$, the distribution functions $F_{\tilde{I}(X)}$ and $F_{I(X)}$ cross three times with $\xi_{1}^{L}=\xi_{1}^{R}=\tilde{d}_{1}, \xi_{2}^{L}=\xi_{2}^{R}=\operatorname{VaR}_{\alpha}(X)-\tilde{d}_{2}+\tilde{d}_{1}$, and $\xi_{3}^{L}=\xi_{3}^{R}=\tilde{d}_{3}-\tilde{d}_{2}+\tilde{d}_{1}$. For any $y \in\left(0, \xi_{1}^{L}\right), F_{\tilde{I}(X)}(y)<F_{I(X)}(y)$. Also, by $(c), \pi_{\tilde{I}(X)}\left(\xi_{2}^{R}\right)=\pi_{I(X)}\left(\xi_{2}^{R}\right)$. Hence, by Theorem 2.2.3, $\tilde{I}(X) \leq_{\mathrm{cx}} I(X)$.

If $I \in \mathcal{I}^{L} \cap \mathcal{I}^{R}$, with parameters $\tau_{1} \in[0, \operatorname{ess} \sup (X)], d_{1} \in\left[0, \tau_{1}\right], d_{2} \in\left[d_{1}, \tau_{1}\right], \tau_{2} \in$ $\left(\tau_{1}, \operatorname{ess} \sup (X)\right], d_{3} \in\left[\tau_{2}, \operatorname{ess} \sup (X)\right]$, and if $d_{3} \leq \operatorname{VaR}_{\alpha}(X)$, then $\tilde{d}_{3}=\operatorname{VaR}_{\alpha}(X)$. In this case, the distribution functions $F_{\tilde{I}(X)}$ and $F_{I(X)}$ cross once with $\xi_{1}^{L}=$ $\xi_{1}^{R}=\tilde{d}_{1}$. For any $y \in\left(d_{1}, \xi_{1}^{L}\right), F_{\tilde{I}(X)}(y)<F_{I(X)}(y)$. Hence, by Theorem 2.2 .3 . $\tilde{I}(X) \leq_{\mathrm{cx}} I(X)$.

If $I \in \mathcal{I}^{L} \cap \mathcal{I}^{R}$, with parameters $\tau_{1} \in[0, \operatorname{ess} \sup (X)], d_{1} \in\left[0, \tau_{1}\right], d_{2} \in\left[d_{1}, \tau_{1}\right], \tau_{2} \in$ $\left(\tau_{1}, \operatorname{ess} \sup (X)\right], d_{3} \in\left[\tau_{2}, \operatorname{ess} \sup (X)\right]$, and if $\tau_{2} \leq \operatorname{VaR}_{\alpha}(X)<d_{3}$, then $\tilde{d}_{3}=d_{3}$. In this case, the distribution functions $F_{\tilde{I}(X)}$ and $F_{I(X)}$ cross once with $\xi_{1}^{L}=\xi_{1}^{R}=\tilde{d}_{1}$. For any $y \in\left(d_{1}, \xi_{1}^{L}\right), F_{\tilde{I}(X)}(y)<F_{I(X)}(y)$. Hence, by Theorem 2.2.3, $\tilde{I}(X) \leq_{\mathrm{cx}}$ $I(X)$.

If $I \in \mathcal{I}^{L} \cap \mathcal{I}^{R}$, with parameters $\tau_{1} \in[0, \operatorname{ess} \sup (X)], d_{1} \in\left[0, \tau_{1}\right], d_{2} \in\left[d_{1}, \tau_{1}\right], \tau_{2} \in$ $\left(\tau_{1}, \operatorname{ess} \sup (X)\right], d_{3} \in\left[\tau_{2}, \operatorname{ess} \sup (X)\right]$, and if $\tau_{1}<\operatorname{VaR}_{\alpha}(X)<\tau_{2}$, the distribution functions $F_{\tilde{I}(X)}$ and $F_{I(X)}$ cross three times with $\xi_{1}^{L}=\xi_{1}^{R}=\tilde{d}_{1}, \xi_{2}^{L}=$ $\xi_{2}^{R}=\operatorname{VaR}_{\alpha}(X)-\tilde{d}_{2}+\tilde{d}_{1}$, and $\xi_{3}^{L}=\xi_{3}^{R}=\tilde{d}_{3}-\tilde{d}_{2}+\tilde{d}_{1}$. For any $y \in\left(d_{1}, \xi_{1}^{L}\right)$, $F_{\tilde{I}(X)}(y)<F_{I(X)}(y)$. Also, by $(c), \pi_{\tilde{I}(X)}\left(\xi_{2}^{R}\right)=\pi_{I(X)}\left(\xi_{2}^{R}\right)$. Hence, by Theorem $2.2 .3 . \tilde{I}(X) \leq_{\mathrm{cx}} I(X)$.

If $I \in \mathcal{I}^{L} \cap \mathcal{I}^{R}$, with parameters $\tau_{1} \in[0, \operatorname{ess} \sup (X)], d_{1} \in\left[0, \tau_{1}\right], d_{2} \in\left[d_{1}, \tau_{1}\right], \tau_{2} \in$ $\left(\tau_{1}, \operatorname{ess} \sup (X)\right], d_{3} \in\left[\tau_{2}, \operatorname{ess} \sup (X)\right]$, and if $d_{2}<\operatorname{VaR}_{\alpha}(X) \leq \tau_{1}$, then $\tilde{d}_{1}=d_{1}$ and 
$\tilde{d}_{2}=d_{2}$. In this case, the distribution functions $F_{\tilde{I}(X)}$ and $F_{I(X)}$ cross once with $\xi_{1}^{L}=\xi_{1}^{R}=\tilde{d}_{3}-d_{2}+d_{1}$. For any $y \in\left(I\left(\tau_{1}\right), \xi_{1}^{L}\right), F_{\tilde{I}(X)}(y)<F_{I(X)}(y)$. Hence, by Theorem 2.2.3, $\tilde{I}(X) \leq_{\mathrm{cx}} I(X)$.

If $I \in \mathcal{I}^{L} \cap \mathcal{I}^{R}$, with parameters $\tau_{1} \in[0, \operatorname{ess} \sup (X)], d_{1} \in\left[0, \tau_{1}\right], d_{2} \in\left[d_{1}, \tau_{1}\right], \tau_{2} \in$ $\left(\tau_{1}, \operatorname{ess} \sup (X)\right], d_{3} \in\left[\tau_{2}, \operatorname{ess} \sup (X)\right]$, and if $d_{1}<\operatorname{VaR}_{\alpha}(X) \leq d_{2}$, then $\tilde{d}_{1}=d_{1}$ and $\tilde{d}_{2}=\operatorname{VaR}_{\alpha}(X)$. In this case, the distribution functions $F_{\tilde{I}(X)}$ and $F_{I(X)}$ cross once with $\xi_{1}^{L}=\xi_{1}^{R}=\tilde{d}_{3}-\operatorname{VaR}_{\alpha}(X)+d_{1}$. For any $y \in\left(d_{1}, \xi_{1}^{L}\right), F_{\tilde{I}(X)}(y)<F_{I(X)}(y)$. Hence, by Theorem 2.2.3, $\tilde{I}(X) \leq_{\mathrm{cx}} I(X)$.

If $I \in \mathcal{I}^{L} \cap \mathcal{I}^{R}$, with parameters $\tau_{1} \in[0, \operatorname{ess} \sup (X)], d_{1} \in\left[0, \tau_{1}\right], d_{2} \in\left[d_{1}, \tau_{1}\right], \tau_{2} \in$ $\left(\tau_{1}, \operatorname{ess} \sup (X)\right], d_{3} \in\left[\tau_{2}, \operatorname{ess} \sup (X)\right]$, and if $0<\operatorname{VaR}_{\alpha}(X) \leq d_{1}$, then $\tilde{d}_{1}=\tilde{d}_{2}=$ $\operatorname{VaR}_{\alpha}(X)$. In this case, the distribution functions $F_{\tilde{I}(X)}$ and $F_{I(X)}$ cross once with $\xi_{1}^{L}=\xi_{1}^{R}=\tilde{d}_{3}$. For any $y \in\left(d_{1}, \xi_{1}^{L}\right), F_{\tilde{I}(X)}(y)<F_{I(X)}(y)$. Hence, by Theorem 2.2.3. $\tilde{I}(X) \leq_{\mathrm{cx}} I(X)$.

Finally, if $I \in \mathcal{I} \backslash\left(\mathcal{I}_{3} \cup \mathcal{I}^{L} \cup \mathcal{I}^{R}\right)$, the distribution functions $F_{\tilde{I}(X)}$ and $F_{I(X)}$ cross three times with $\xi_{1}^{L}=\xi_{1}^{R}=\tilde{d}_{1}, \xi_{2}^{L}=\xi_{2}^{R}=\operatorname{VaR}_{\alpha}(X)-\tilde{d}_{2}+\tilde{d}_{1}$, and $\xi_{3}^{L}=\xi_{3}^{R}=\tilde{d}_{3}-\tilde{d}_{2}+\tilde{d}_{1}$. For any $y \in\left(0, \xi_{1}^{L}\right), F_{\tilde{I}(X)}(y)<F_{I(X)}(y)$. Also, by $(c)$, $\pi_{\tilde{I}(X)}\left(\xi_{2}^{R}\right)=\pi_{I(X)}\left(\xi_{2}^{R}\right)$. Hence, by Theorem 2.2.3. $\tilde{I}(X) \leq_{\mathrm{cx}} I(X)$.

Therefore, in any case, $\tilde{I}(X) \leq_{\mathrm{cx}} I(X)$.

Theorem 2.2.5. For any $I \in \mathcal{I}_{3}$, there exists an $\tilde{I} \in \mathcal{I}_{3}$, with parameters $\tilde{d}_{1} \in$ $[0, \operatorname{ess} \sup (X)], \tilde{d}_{2} \in\left[\tilde{d}_{1}, \operatorname{ess} \sup (X)\right], \tilde{d}_{3} \in\left[\tilde{d}_{2}, \operatorname{ess} \sup (X)\right]$, such that

(i) $\operatorname{VaR}_{\alpha}(X) \in\left[\tilde{d}_{2}, \tilde{d}_{3}\right]$;

(ii) $\mathbb{E}[\tilde{I}(X)]=\mathbb{E}[I(X)]$;

(iii) $\operatorname{VaR}_{\alpha}(\tilde{I}(X))=\operatorname{VaR}_{\alpha}(I(X))$;

(iv) $T \operatorname{Va} R_{\alpha}(\tilde{I}(X))=T \operatorname{Va} R_{\alpha}(I(X))$; 
(v) $\tilde{I}(X) \leq_{c x} I(X)$

Proof. Let $I \in \mathcal{I}_{3}$, with parameters $d_{1} \in[0, \operatorname{ess} \sup (X)], d_{2} \in\left[d_{1}, \operatorname{ess} \sup (X)\right], d_{3} \in$ $\left[d_{2}, \operatorname{ess} \sup (X)\right]$. The distribution function of $I(X)$ is given by

$$
F_{I(X)}(y)= \begin{cases}F_{X}(y), & \forall y \in\left[0, d_{1}\right), \\ F_{X}\left(y+d_{2}-d_{1}\right), & \forall y \in\left[d_{1}, d_{3}-d_{2}+d_{1}\right), \\ 1, & \forall y \in\left[d_{3}-d_{2}+d_{1}, \operatorname{ess} \sup (X)\right] .\end{cases}
$$

If $\operatorname{VaR}_{\alpha}(X) \in\left[d_{2}, d_{3}\right]$, the proof completes by defining $\tilde{I}=I$.

If $d_{3}<\operatorname{VaR}_{\alpha}(X)$, by Intermediate Value Theorem, there exist $\tilde{d}_{1} \in\left[0, \operatorname{VaR}_{\alpha}(X)\right], \tilde{d}_{2} \in$ $\left[\tilde{d}_{1}, \operatorname{VaR}_{\alpha}(X)\right]$, such that

(a) $\mathbb{E}\left[X-\left(X-\tilde{d}_{1}\right)_{+}+\left(X-\tilde{d}_{2}\right)_{+}-\left(X-\operatorname{VaR}_{\alpha}(X)\right)_{+}\right]=\mathbb{E}[I(X)]$;

(b) $\operatorname{VaR}_{\alpha}(X)-\tilde{d}_{2}+\tilde{d}_{1}=I\left(\operatorname{VaR}_{\alpha}(X)\right)$.

Define

$$
\tilde{I}(x)=x-\left(x-\tilde{d}_{1}\right)_{+}+\left(x-\tilde{d}_{2}\right)_{+}-\left(x-\operatorname{VaR}_{\alpha}(X)\right)_{+}, \quad \forall x \in[0, \operatorname{ess} \sup (X)] .
$$

Then, $\tilde{I} \in \mathcal{I}_{3}$, with $\tilde{d}_{3}=\operatorname{VaR}_{\alpha}(X) ;(a)$ is equivalent to that

$$
\mathbb{E}[\tilde{I}(X)]=\mathbb{E}[I(X)]
$$

Since both $I$ and $\tilde{I}$ are non-decreasing, by (b),

$$
\begin{aligned}
\operatorname{VaR}_{\alpha}(\tilde{I}(X)) & =\tilde{I}\left(\operatorname{VaR}_{\alpha}(X)\right) \\
& =\operatorname{VaR}_{\alpha}(X)-\tilde{d}_{2}+\tilde{d}_{1} \\
& =I\left(\operatorname{VaR}_{\alpha}(X)\right) \\
& =\operatorname{VaR}_{\alpha}(I(X)) ;
\end{aligned}
$$


Since $\operatorname{VaR}_{\alpha}(\tilde{I}(X))=\operatorname{VaR}_{\alpha}(I(X))$

$$
\begin{aligned}
\operatorname{TVaR}_{\alpha}(\tilde{I}(X)) & =\operatorname{VaR}_{\alpha}(\tilde{I}(X))+\frac{1}{1-\alpha} \pi_{\tilde{I}(X)}\left(\operatorname{VaR}_{\alpha}(\tilde{I}(X))\right) \\
& =\operatorname{VaR}_{\alpha}(\tilde{I}(X))+\frac{1}{1-\alpha} \mathbb{E}\left[\left(\tilde{I}(X)-\operatorname{VaR}_{\alpha}(\tilde{I}(X))\right)_{+}\right] \\
& =\operatorname{VaR}_{\alpha}(\tilde{I}(X)) \\
& =\operatorname{VaR}_{\alpha}(I(X)) \\
& =\operatorname{VaR}_{\alpha}(I(X))+\frac{1}{1-\alpha} \mathbb{E}\left[\left(I(X)-\operatorname{VaR}_{\alpha}(I(X))\right)_{+}\right] \\
& =\operatorname{VaR}_{\alpha}(I(X))+\frac{1}{1-\alpha} \pi_{I(X)}\left(\operatorname{VaR}_{\alpha}(I(X))\right) \\
& =\operatorname{TVaR}_{\alpha}(I(X)) ;
\end{aligned}
$$

The distribution function of $\tilde{I}(X)$ is given by

$$
F_{\tilde{I}(X)}(y)= \begin{cases}F_{X}(y), & \forall y \in\left[0, \tilde{d}_{1}\right), \\ F_{X}\left(y+\tilde{d}_{2}-\tilde{d}_{1}\right), & \forall y \in\left[\tilde{d}_{1}, \operatorname{VaR}_{\alpha}(X)-\tilde{d}_{2}+\tilde{d}_{1}\right), \\ 1, & \forall y \in\left[\operatorname{VaR}_{\alpha}(X)-\tilde{d}_{2}+\tilde{d}_{1}, \operatorname{ess} \sup (X)\right] .\end{cases}
$$

In this case, the distribution functions $F_{\tilde{I}(X)}$ and $F_{I(X)}$ cross once with $\xi_{1}^{L}=$ $\xi_{1}^{R}=\tilde{d}_{1}$. For any $y \in\left(d_{1}, \xi_{1}^{L}\right), F_{\tilde{I}(X)}(y)<F_{I(X)}(y)$. Hence, by Theorem 2.2.3. $\tilde{I}(X) \leq_{\mathrm{cx}} I(X)$.

If $d_{1}<\operatorname{VaR}_{\alpha}(X)<d_{2}$, by Intermediate Value Theorem, there exists $\tilde{d}_{3} \in$ $\left[\operatorname{VaR}_{\alpha}(X), \operatorname{ess} \sup (X)\right]$, such that

(a) $\mathbb{E}\left[X-\left(X-d_{1}\right)_{+}+\left(X-\operatorname{VaR}_{\alpha}(X)\right)_{+}-\left(X-\tilde{d}_{3}\right)_{+}\right]=\mathbb{E}[I(X)]$.

Define

$$
\tilde{I}(x)=x-\left(x-d_{1}\right)_{+}+\left(x-\operatorname{VaR}_{\alpha}(X)\right)_{+}-\left(x-\tilde{d}_{3}\right)_{+}, \quad \forall x \in[0, \operatorname{ess} \sup (X)]
$$

Then, $\tilde{I} \in \mathcal{I}_{3}$, with $\tilde{d}_{1}=d_{1}$ and $\tilde{d}_{2}=\operatorname{VaR}_{\alpha}(X) ;(a)$ is equivalent to that

$$
\mathbb{E}[\tilde{I}(X)]=\mathbb{E}[I(X)]
$$


from which, we have

$$
\begin{aligned}
\mathbb{E}\left[\left(\tilde{I}(X)-d_{1}\right)_{+}\right] & =\mathbb{E}\left[\tilde{I}(X)-d_{1}\right]-\mathbb{E}\left[\left(\tilde{I}(X)-d_{1}\right) \mathbb{1}_{\left\{\tilde{I}(X)<d_{1}\right\}}\right] \\
& =\mathbb{E}\left[I(X)-d_{1}\right]-\mathbb{E}\left[\left(X-d_{1}\right) \mathbb{1}_{\left\{X<d_{1}\right\}}\right] \\
& =\mathbb{E}\left[I(X)-d_{1}\right]-\mathbb{E}\left[\left(I(X)-d_{1}\right) \mathbb{1}_{\left\{I(X)<d_{1}\right\}}\right] \\
& =\mathbb{E}\left[\left(I(X)-d_{1}\right)_{+}\right]
\end{aligned}
$$

Since both $I$ and $\tilde{I}$ are non-decreasing,

$$
\begin{aligned}
\operatorname{VaR}_{\alpha}(\tilde{I}(X)) & =\tilde{I}\left(\operatorname{VaR}_{\alpha}(X)\right) \\
& =d_{1} \\
& =I\left(\operatorname{VaR}_{\alpha}(X)\right) \\
& =\operatorname{VaR}_{\alpha}(I(X)) ;
\end{aligned}
$$

Since $\operatorname{VaR}_{\alpha}(\tilde{I}(X))=\operatorname{VaR}_{\alpha}(I(X))=d_{1}$ and $\mathbb{E}\left[\left(\tilde{I}(X)-d_{1}\right)_{+}\right]=\mathbb{E}\left[\left(I(X)-d_{1}\right)_{+}\right]$,

$$
\begin{aligned}
\operatorname{TVaR}_{\alpha}(\tilde{I}(X)) & =\operatorname{VaR}_{\alpha}(\tilde{I}(X))+\frac{1}{1-\alpha} \pi_{\tilde{I}(X)}\left(\operatorname{VaR}_{\alpha}(\tilde{I}(X))\right) \\
& =\operatorname{VaR}_{\alpha}(\tilde{I}(X))+\frac{1}{1-\alpha} \mathbb{E}\left[\left(\tilde{I}(X)-\operatorname{VaR}_{\alpha}(\tilde{I}(X))\right)_{+}\right] \\
& =\operatorname{VaR}_{\alpha}(\tilde{I}(X))+\frac{1}{1-\alpha} \mathbb{E}\left[\left(\tilde{I}(X)-d_{1}\right)_{+}\right] \\
& =\operatorname{VaR}_{\alpha}(I(X))+\frac{1}{1-\alpha} \mathbb{E}\left[\left(I(X)-d_{1}\right)_{+}\right] \\
& =\operatorname{VaR}_{\alpha}(I(X))+\frac{1}{1-\alpha} \mathbb{E}\left[\left(I(X)-\operatorname{VaR}_{\alpha}(I(X))\right)_{+}\right] \\
& =\operatorname{VaR}_{\alpha}(I(X))+\frac{1}{1-\alpha} \pi_{I(X)}\left(\operatorname{VaR}_{\alpha}(I(X))\right) \\
& =\operatorname{TVaR}_{\alpha}(I(X)) ;
\end{aligned}
$$

The distribution function of $\tilde{I}(X)$ is given by

$$
F_{\tilde{I}(X)}(y)= \begin{cases}F_{X}(y), & \forall y \in\left[0, d_{1}\right), \\ F_{X}\left(y+\operatorname{VaR}_{\alpha}(X)-d_{1}\right), & \forall y \in\left[d_{1}, \tilde{d}_{3}-\operatorname{VaR}_{\alpha}(X)+d_{1}\right), \\ 1, & \forall y \in\left[\tilde{d}_{3}-\operatorname{VaR}_{\alpha}(X)+d_{1}, \operatorname{ess} \sup (X)\right] .\end{cases}
$$


In this case, the distribution functions $F_{\tilde{I}(X)}$ and $F_{I(X)}$ cross once with $\xi_{1}^{L}=\xi_{1}^{R}=$ $\tilde{d}_{3}-\operatorname{VaR}_{\alpha}(X)+d_{1}$. For any $y \in\left(d_{1}, \xi_{1}^{L}\right), F_{\tilde{I}(X)}(y)<F_{I(X)}(y)$. Hence, by Theorem 2.2.3. $\tilde{I}(X) \leq_{\mathrm{cx}} I(X)$.

Finally, if $0<\operatorname{VaR}_{\alpha}(X) \leq d_{1}$, by Intermediate Value Theorem, there exists $\tilde{d}_{3} \in\left[d_{1}, \operatorname{ess} \sup (X)\right]$, such that

(a) $\mathbb{E}\left[X-\left(X-\tilde{d}_{3}\right)_{+}\right]=\mathbb{E}[I(X)]$.

Define

$$
\tilde{I}(x)=x-\left(x-\tilde{d}_{3}\right)_{+}, \quad \forall x \in[0, \operatorname{ess} \sup (X)] .
$$

Then, $\tilde{I} \in \mathcal{I}_{3}$, with $\tilde{d}_{1}=\tilde{d}_{2}=\operatorname{VaR}_{\alpha}(X) ;(a)$ is equivalent to that

$$
\mathbb{E}[\tilde{I}(X)]=\mathbb{E}[I(X)],
$$

from which, we have

$$
\begin{aligned}
\mathbb{E}\left[\left(\tilde{I}(X)-\operatorname{VaR}_{\alpha}(X)\right)_{+}\right]= & \mathbb{E}\left[\tilde{I}(X)-\operatorname{VaR}_{\alpha}(X)\right] \\
& -\mathbb{E}\left[\left(\tilde{I}(X)-\operatorname{VaR}_{\alpha}(X)\right) \mathbb{1}_{\left\{\tilde{I}(X)<\operatorname{VaR}_{\alpha}(X)\right\}}\right] \\
= & \mathbb{E}\left[I(X)-\operatorname{VaR}_{\alpha}(X)\right]-\mathbb{E}\left[\left(X-\operatorname{VaR}_{\alpha}(X)\right) \mathbb{1}_{\left\{X<\operatorname{VaR}_{\alpha}(X)\right\}}\right] \\
= & \mathbb{E}\left[I(X)-\operatorname{VaR}_{\alpha}(X)\right] \\
& -\mathbb{E}\left[\left(I(X)-\operatorname{VaR}_{\alpha}(X)\right) \mathbb{1}_{\left\{I(X)<\operatorname{VaR}_{\alpha}(X)\right\}}\right] \\
= & \mathbb{E}\left[\left(I(X)-\operatorname{VaR}_{\alpha}(X)\right)_{+}\right]
\end{aligned}
$$

Since both $I$ and $\tilde{I}$ are non-decreasing,

$$
\begin{aligned}
\operatorname{VaR}_{\alpha}(\tilde{I}(X)) & =\tilde{I}\left(\operatorname{VaR}_{\alpha}(X)\right) \\
& =\operatorname{VaR}_{\alpha}(X) \\
& =I\left(\operatorname{VaR}_{\alpha}(X)\right) \\
& =\operatorname{VaR}_{\alpha}(I(X)) ;
\end{aligned}
$$


Since $\operatorname{VaR}_{\alpha}(\tilde{I}(X))=\operatorname{VaR}_{\alpha}(I(X))=\operatorname{VaR}_{\alpha}(X)$ and $\mathbb{E}\left[\left(\tilde{I}(X)-\operatorname{VaR}_{\alpha}(X)\right)_{+}\right]=$ $\mathbb{E}\left[\left(I(X)-\operatorname{VaR}_{\alpha}(X)\right)_{+}\right]$

$$
\begin{aligned}
\operatorname{TVaR}_{\alpha}(\tilde{I}(X)) & =\operatorname{VaR}_{\alpha}(\tilde{I}(X))+\frac{1}{1-\alpha} \pi_{\tilde{I}(X)}\left(\operatorname{VaR}_{\alpha}(\tilde{I}(X))\right) \\
& =\operatorname{VaR}_{\alpha}(\tilde{I}(X))+\frac{1}{1-\alpha} \mathbb{E}\left[\left(\tilde{I}(X)-\operatorname{VaR}_{\alpha}(\tilde{I}(X))\right)_{+}\right] \\
& =\operatorname{VaR}_{\alpha}(\tilde{I}(X))+\frac{1}{1-\alpha} \mathbb{E}\left[\left(\tilde{I}(X)-\operatorname{VaR}_{\alpha}(X)\right)_{+}\right] \\
& =\operatorname{VaR}_{\alpha}(I(X))+\frac{1}{1-\alpha} \mathbb{E}\left[\left(I(X)-\operatorname{VaR}_{\alpha}(X)\right)_{+}\right] \\
& =\operatorname{VaR}_{\alpha}(I(X))+\frac{1}{1-\alpha} \mathbb{E}\left[\left(I(X)-\operatorname{VaR}_{\alpha}(I(X))\right)_{+}\right] \\
& =\operatorname{VaR}_{\alpha}(I(X))+\frac{1}{1-\alpha} \pi_{I(X)}\left(\operatorname{VaR}_{\alpha}(I(X))\right) \\
& =\operatorname{TVaR}_{\alpha}(I(X)) ;
\end{aligned}
$$

The distribution function of $\tilde{I}(X)$ is given by

$$
F_{\tilde{I}(X)}(y)= \begin{cases}F_{X}(y), & \forall y \in\left[0, \tilde{d}_{3}\right), \\ 1, & \forall y \in\left[\tilde{d}_{3}, \operatorname{ess} \sup (X)\right] .\end{cases}
$$

In this case, the distribution functions $F_{\tilde{I}(X)}$ and $F_{I(X)}$ cross once with $\xi_{1}^{L}=$ $\xi_{1}^{R}=\tilde{d}_{3}$. For any $y \in\left(d_{1}, \xi_{1}^{L}\right), F_{\tilde{I}(X)}(y)<F_{I(X)}(y)$. Hence, by Theorem 2.2.3. $\tilde{I}(X) \leq_{\mathrm{cx}} I(X)$.

\subsubsection{Solution of Problem (2.2.3}

After showing Theorem 2.2.4 and 2.2.5, we are ready to solve Problem 2.2.3.

Theorem 2.2.6. The optimal ceded loss function of Problem 2.2.3 is given by

$$
I^{*}(x)=x-\left(x-d_{1}^{*}\right)_{+}+\left(x-d_{2}^{*}\right)_{+}-\left(x-d_{3}^{*}\right)_{+}, \quad \forall x \in[0, \operatorname{ess} \sup (X)]
$$

for some $d_{1}^{*} \in\left[0, \operatorname{VaR}_{\alpha}(X)\right], d_{2}^{*} \in\left[d_{1}^{*}, \operatorname{VaR}_{\alpha}(X)\right], d_{3}^{*} \in\left[\operatorname{Va} R_{\alpha}(X), \operatorname{ess} \sup (X)\right]$.

Before proving the above statement, we discuss its essence. The theorem depicts that the optimal solutions of any optimal reinsurance design problems in 
the form of Problem (2.2.3) must lie in the class $\mathcal{I}_{3}$ with $d_{2}^{*} \leq \operatorname{VaR}_{\alpha}(X) \leq d_{3}^{*}$. However, the theorem does not specify the relations between the optimal points $d_{1}^{*}, d_{2}^{*}$, and $d_{3}^{*}$ and the objective function $G$ and the premium principle $g$, as well as the effect on the optimal points by the budget $\pi$. To determine the optimal points $d_{1}^{*}, d_{2}^{*}$, and $d_{3}^{*}$, one has to specify the functions $G$ and $g$ and the constant $\pi$. The importance of the theorem is that it reduces any original infinite dimensional problem in $(2.2 .3)$ to a finite dimensional problem.

Proof. By the definition of $T_{I}(X)$ in 2.1.1), the translation invariance of VaR and TVaR, and the comonotonic additivity of VaR and TVaR, the objective in Problem 2.2.3 can be rewritten as

$$
\begin{gathered}
G\left(\mathbb{E}[X]-\mathbb{E}[I(X)]+g\left(\mathbb{E}[I(X)], v_{\mathrm{cx}}(I(X))\right)\right. \\
\operatorname{VaR}_{\alpha}(X)-\operatorname{VaR}_{\alpha}(I(X))+g\left(\mathbb{E}[I(X)], v_{\mathrm{cx}}(I(X))\right) \\
\left.\operatorname{TVaR}_{\alpha}(X)-\mathrm{TVaR}_{\alpha}(I(X))+g\left(\mathbb{E}[I(X)], v_{\mathrm{cx}}(I(X))\right)\right) .
\end{gathered}
$$

Let $I \in \mathcal{I}$ such that

$$
g\left(\mathbb{E}[I(X)], v_{\mathrm{cx}}(I(X))\right) \leq \pi
$$

By Theorem 2.2.4, if $I \in \mathcal{I} \backslash \mathcal{I}_{3}$, or Theorem 2.2.5, if $I \in \mathcal{I}_{3}$, there exists $\tilde{I} \in \mathcal{I}_{3}$, with parameters $\tilde{d}_{1} \in\left[0, \operatorname{VaR}_{\alpha}(X)\right], \tilde{d}_{2} \in\left[\tilde{d}_{1}, \operatorname{VaR}_{\alpha}(X)\right], \tilde{d}_{3} \in\left[\operatorname{VaR}_{\alpha}(X), \operatorname{ess} \sup (X)\right]$, such that

(i) $\mathbb{E}[\tilde{I}(X)]=\mathbb{E}[I(X)]$;

(ii) $\operatorname{VaR}_{\alpha}(\tilde{I}(X))=\operatorname{VaR}_{\alpha}(I(X))$;

(iii) $\operatorname{TVaR}_{\alpha}(\tilde{I}(X))=\operatorname{TVaR}_{\alpha}(I(X))$;

(iv) $\tilde{I}(X) \leq_{\mathrm{cx}} I(X)$. 
Since $v_{c x}$ is a convex order preserving functional, (iv) implies that

$$
v_{\mathrm{cx}}(\tilde{I}(X)) \leq v_{\mathrm{cx}}(I(X))
$$

Since $G$ and $g$ are non-decreasing functions,

$$
\begin{gathered}
G\left(\mathbb{E}[X]-\mathbb{E}[\tilde{I}(X)]+g\left(\mathbb{E}[\tilde{I}(X)], v_{\mathrm{cx}}(\tilde{I}(X))\right)\right. \\
\operatorname{VaR}_{\alpha}(X)-\operatorname{VaR}_{\alpha}(\tilde{I}(X))+g\left(\mathbb{E}[\tilde{I}(X)], v_{\mathrm{cx}}(\tilde{I}(X))\right), \\
\left.\operatorname{TVaR}_{\alpha}(X)-\operatorname{TVaR}_{\alpha}(\tilde{I}(X))+g\left(\mathbb{E}[\tilde{I}(X)], v_{\mathrm{cx}}(\tilde{I}(X))\right)\right) \\
\leq G\left(\mathbb{E}[X]-\mathbb{E}[I(X)]+g\left(\mathbb{E}[I(X)], v_{\mathrm{cx}}(I(X))\right),\right. \\
\operatorname{VaR}_{\alpha}(X)-\operatorname{VaR}_{\alpha}(I(X))+g\left(\mathbb{E}[I(X)], v_{\mathrm{cx}}(I(X))\right), \\
\left.\operatorname{TVaR}_{\alpha}(X)-\operatorname{TVaR}_{\alpha}(I(X))+g\left(\mathbb{E}[I(X)], v_{\mathrm{cx}}(I(X))\right)\right) .
\end{gathered}
$$

Finally, since $g$ is a non-decreasing function,

$$
g\left(\mathbb{E}[\tilde{I}(X)], v_{\mathrm{cx}}(\tilde{I}(X))\right) \leq g\left(\mathbb{E}[I(X)], v_{\mathrm{cx}}(I(X))\right) \leq \pi
$$

\subsubsection{Solutions of Sub-problems of Problem (2.2.3)}

We further study the following sub-problems of Problem 2.2.3):

$$
\begin{cases}\inf _{I \in \mathcal{I}} & G_{E, T V}\left(\mathbb{E}\left[T_{I}(X)\right], \operatorname{TVaR}_{\alpha}\left(T_{I}(X)\right)\right), \\ \text { s.t. } & g\left(\mathbb{E}[I(X)], v_{\mathrm{cx}}(I(X))\right) \leq \pi\end{cases}
$$

where $G_{E, T V}$ is a real-valued function on $\mathbb{R}_{+}^{2}$, non-decreasing in both arguments;

$$
\begin{cases}\inf _{I \in \mathcal{I}} & G_{E, V}\left(\mathbb{E}\left[T_{I}(X)\right], \operatorname{VaR}_{\alpha}\left(T_{I}(X)\right)\right), \\ \text { s.t. } & g\left(\mathbb{E}[I(X)], v_{\mathrm{cx}}(I(X))\right) \leq \pi,\end{cases}
$$


where $G_{E, V}$ is a real-valued function on $\mathbb{R}_{+}^{2}$, non-decreasing in both arguments;

$$
\begin{cases}\inf _{I \in \mathcal{I}} & G_{E}\left(\mathbb{E}\left[T_{I}(X)\right]\right), \\ \text { s.t. } & g\left(\mathbb{E}[I(X)], v_{\mathrm{cx}}(I(X))\right) \leq \pi,\end{cases}
$$

where $G_{E}$ is a real-valued non-decreasing function on $\mathbb{R}_{+}$.

Thanks to Theorem 2.2.6, solving Problems 2.2.4, 2.2.5), and 2.2.6, becomes less sophisticated.

Corollary 2.2.7 (Extension of Chi (2012b)). The optimal ceded loss function of Problem 2.2.4 is given by

$$
I^{*}(x)=x-\left(x-d_{1}^{*}\right)_{+}+\left(x-d_{2}^{*}\right)_{+}-\left(x-d_{3}^{*}\right)_{+}, \quad \forall x \in[0, \operatorname{ess} \sup (X)]
$$

for some $d_{1}^{*} \in\left[0, \operatorname{VaR}_{\alpha}(X)\right], d_{2}^{*} \in\left[d_{1}^{*}, \operatorname{Va} R_{\alpha}(X)\right], d_{3}^{*} \in\left[\operatorname{Va} R_{\alpha}(X), \operatorname{ess} \sup (X)\right]$.

Proof. This is a direct consequence of Theorem 2.2.6.

Corollary 2.2.8 (Extension of Chi (2012b)). The optimal ceded loss function of Problem (2.2.5) is given by either

$$
I^{*}(x)=x-\left(x-d_{1}^{*}\right)_{+}+\left(x-d_{2}^{*}\right)_{+}-\left(x-V a R_{\alpha}(X)\right)_{+}, \quad \forall x \in[0, \operatorname{ess} \sup (X)]
$$

for some $d_{1}^{*} \in\left[0, \operatorname{VaR}_{\alpha}(X)\right], d_{2}^{*} \in\left[d_{1}^{*}, \operatorname{VaR}_{\alpha}(X)\right]$, or

$$
I^{* *}(x)=x-\left(x-d^{* *}\right), \quad \forall x \in[0, \operatorname{ess} \sup (X)]
$$

for some $d^{* *} \in[0, \operatorname{ess} \sup (X)]$.

Proof. By the definition of $T_{I}(X)$ in (2.1.1), the translation invariance of VaR, and the comonotonic additivity of VaR, the objective in Problem 2.2.5 can be rewritten as

$$
\begin{aligned}
G_{E, V}( & \mathbb{E}[X]-\mathbb{E}[I(X)]+g\left(\mathbb{E}[I(X)], v_{\mathrm{cx}}(I(X))\right) \\
& \left.\operatorname{VaR}_{\alpha}(X)-\operatorname{VaR}_{\alpha}(I(X))+g\left(\mathbb{E}[I(X)], v_{\mathrm{cx}}(I(X))\right)\right) .
\end{aligned}
$$


By Theorem 2.2.6, it suffices to consider any $I \in \mathcal{I}_{3}$, with parameters $d_{1} \in$ $\left[0, \operatorname{VaR}_{\alpha}(X)\right], d_{2} \in\left[d_{1}, \operatorname{VaR}_{\alpha}(X)\right], d_{3} \in\left[\operatorname{VaR}_{\alpha}(X), \operatorname{ess} \sup (X)\right]$, such that

$$
g\left(\mathbb{E}[I(X)], v_{\mathrm{cx}}(I(X))\right) \leq \pi
$$

If

$$
\int_{d_{1}}^{I\left(\operatorname{VaR}_{\alpha}(X)\right)}\left(F_{I(X)}(y)-F_{X}(y)\right) d y>\int_{I\left(\operatorname{VaR}_{\alpha}(X)\right)}^{d_{3}-d_{2}+d_{1}}\left(1-F_{I(X)}(y)\right) d y,
$$

by Intermediate Value Theorem, there exist $\tilde{d}_{1} \in\left[0, \operatorname{VaR}_{\alpha}(X)\right], \tilde{d}_{2} \in\left[\tilde{d}_{1}, \operatorname{VaR}_{\alpha}(X)\right]$, such that

(a) $\mathbb{E}\left[X-\left(X-\tilde{d}_{1}\right)_{+}+\left(X-\tilde{d}_{2}\right)_{+}-\left(X-\operatorname{VaR}_{\alpha}(X)\right)_{+}\right]=\mathbb{E}[I(X)]$;

(b) $\operatorname{VaR}_{\alpha}(X)-\tilde{d}_{2}+\tilde{d}_{1}=I\left(\operatorname{VaR}_{\alpha}(X)\right)$.

Define

$$
\tilde{I}(x)=x-\left(x-\tilde{d}_{1}\right)_{+}+\left(x-\tilde{d}_{2}\right)_{+}-\left(x-\operatorname{VaR}_{\alpha}(X)\right)_{+}, \quad \forall x \in[0, \operatorname{ess} \sup (X)] .
$$

Then, $(a)$ is equivalent to that

$$
\mathbb{E}[\tilde{I}(X)]=\mathbb{E}[I(X)]
$$

Since both $I$ and $\tilde{I}$ are non-decreasing, by (b),

$$
\begin{aligned}
\operatorname{VaR}_{\alpha}(\tilde{I}(X)) & =\tilde{I}\left(\operatorname{VaR}_{\alpha}(X)\right) \\
& =\operatorname{VaR}_{\alpha}(X)-\tilde{d}_{2}+\tilde{d}_{1} \\
& =I\left(\operatorname{VaR}_{\alpha}(X)\right) \\
& =\operatorname{VaR}_{\alpha}(I(X)) ;
\end{aligned}
$$

The distribution function of $\tilde{I}(X)$ is given by

$$
F_{\tilde{I}(X)}(y)= \begin{cases}F_{X}(y), & \forall y \in\left[0, \tilde{d}_{1}\right), \\ F_{X}\left(y+\tilde{d}_{2}-\tilde{d}_{1}\right), & \forall y \in\left[\tilde{d}_{1}, \operatorname{VaR}_{\alpha}(X)-\tilde{d}_{2}+\tilde{d}_{1}\right), \\ 1, & \forall y \in\left[\operatorname{VaR}_{\alpha}(X)-\tilde{d}_{2}+\tilde{d}_{1}, \operatorname{ess} \sup (X)\right] .\end{cases}
$$


In this case, the distribution functions $F_{\tilde{I}(X)}$ and $F_{I(X)}$ cross once with $\xi_{1}^{L}=\tilde{d}_{1}$ and $\xi_{1}^{R}=\operatorname{VaR}_{\alpha}(X)-\tilde{d}_{2}+\tilde{d}_{1}$. For any $y \in\left(d_{1}, \xi_{1}^{L}\right), F_{\tilde{I}(X)}(y)<F_{I(X)}(y)$. Hence, by Theorem 2.2.3. $\tilde{I}(X) \leq_{\mathrm{cx}} I(X)$. Since $v_{c x}$ is a convex order preserving functional,

$$
v_{\mathrm{cx}}(\tilde{I}(X)) \leq v_{\mathrm{cx}}(I(X))
$$

Since $G_{E, V}$ and $g$ are non-decreasing functions,

$$
\begin{aligned}
G_{E, V}( & \mathbb{E}[X]-\mathbb{E}[\tilde{I}(X)]+g\left(\mathbb{E}[\tilde{I}(X)], v_{\mathrm{cx}}(\tilde{I}(X))\right), \\
& \left.\operatorname{VaR}_{\alpha}(X)-\operatorname{VaR}_{\alpha}(\tilde{I}(X))+g\left(\mathbb{E}[\tilde{I}(X)], v_{\mathrm{cx}}(\tilde{I}(X))\right)\right) \\
\leq G_{E, V}( & \mathbb{E}[X]-\mathbb{E}[I(X)]+g\left(\mathbb{E}[I(X)], v_{\mathrm{cx}}(I(X))\right), \\
& \left.\operatorname{VaR}_{\alpha}(X)-\operatorname{VaR}_{\alpha}(I(X))+g\left(\mathbb{E}[I(X)], v_{\mathrm{cx}}(I(X))\right)\right) .
\end{aligned}
$$

Finally, since $g$ is a non-decreasing function,

$$
g\left(\mathbb{E}[\tilde{I}(X)], v_{\mathrm{cx}}(\tilde{I}(X))\right) \leq g\left(\mathbb{E}[I(X)], v_{\mathrm{cx}}(I(X))\right) \leq \pi .
$$

If

$$
\int_{d_{1}}^{I\left(\operatorname{VaR}_{\alpha}(X)\right)}\left(F_{I(X)}(y)-F_{X}(y)\right) d y \leq \int_{I\left(\operatorname{VaR}_{\alpha}(X)\right)}^{d_{3}-d_{2}+d_{1}}\left(1-F_{I(X)}(y)\right) d y,
$$

by Intermediate Value Theorem, there exist $\tilde{d} \in[0, \operatorname{ess} \sup (X)]$ such that

(a) $\mathbb{E}\left[X-(X-\tilde{d})_{+}\right]=\mathbb{E}[I(X)]$

Define

$$
\tilde{I}(x)=x-(x-\tilde{d})_{+}, \quad \forall x \in[0, \operatorname{ess} \sup (X)] .
$$

Then, $(a)$ is equivalent to that

$$
\mathbb{E}[\tilde{I}(X)]=\mathbb{E}[I(X)]
$$


Since both $I$ and $\tilde{I}$ are non-decreasing,

$$
\begin{aligned}
\operatorname{VaR}_{\alpha}(\tilde{I}(X)) & =\tilde{I}\left(\operatorname{VaR}_{\alpha}(X)\right) \\
& \geq I\left(\operatorname{VaR}_{\alpha}(X)\right) \\
& =\operatorname{VaR}_{\alpha}(I(X))
\end{aligned}
$$

The distribution function of $\tilde{I}(X)$ is given by

$$
F_{\tilde{I}(X)}(y)= \begin{cases}F_{X}(y), & \forall y \in[0, \tilde{d}) \\ 1, & \forall y \in[\tilde{d}, \operatorname{ess} \sup (X)] .\end{cases}
$$

In this case, the distribution functions $F_{\tilde{I}(X)}$ and $F_{I(X)}$ cross once with $\xi_{1}^{L}=$ $\xi_{1}^{R}=\tilde{d}$. For any $y \in\left(d_{1}, \xi_{1}^{L}\right), F_{\tilde{I}(X)}(y)<F_{I(X)}(y)$. Hence, by Theorem 2.2.3. $\tilde{I}(X) \leq_{\mathrm{cx}} I(X)$. Since $v_{c x}$ is a convex order preserving functional,

$$
v_{\mathrm{cx}}(\tilde{I}(X)) \leq v_{\mathrm{cx}}(I(X))
$$

Since $G_{E, V}$ and $g$ are non-decreasing functions,

$$
\begin{gathered}
G_{E, V}\left(\mathbb{E}[X]-\mathbb{E}[\tilde{I}(X)]+g\left(\mathbb{E}[\tilde{I}(X)], v_{\mathrm{cx}}(\tilde{I}(X))\right)\right. \\
\left.\operatorname{VaR}_{\alpha}(X)-\operatorname{VaR}_{\alpha}(\tilde{I}(X))+g\left(\mathbb{E}[\tilde{I}(X)], v_{\mathrm{cx}}(\tilde{I}(X))\right)\right) \\
\leq G_{E, V}\left(\mathbb{E}[X]-\mathbb{E}[I(X)]+g\left(\mathbb{E}[I(X)], v_{\mathrm{cx}}(I(X))\right),\right. \\
\left.\operatorname{VaR}_{\alpha}(X)-\operatorname{VaR}_{\alpha}(I(X))+g\left(\mathbb{E}[I(X)], v_{\mathrm{cx}}(I(X))\right)\right) .
\end{gathered}
$$

Finally, since $g$ is a non-decreasing function,

$$
g\left(\mathbb{E}[\tilde{I}(X)], v_{\mathrm{cx}}(\tilde{I}(X))\right) \leq g\left(\mathbb{E}[I(X)], v_{\mathrm{cx}}(I(X))\right) \leq \pi
$$

Corollary 2.2.9. The optimal ceded loss function of Problem (2.2.6) is given by

$$
I^{*}(x)=x-\left(x-d^{*}\right), \quad \forall x \in[0, \operatorname{ess} \sup (X)]
$$

for some $d^{*} \in[0, \operatorname{ess} \sup (X)]$. 
Proof. By the definition of $T_{I}(X)$ in 2.1.1, the objective in Problem 2.2.6 can be rewritten as

$$
G_{E}\left(\mathbb{E}[X]-\mathbb{E}[I(X)]+g\left(\mathbb{E}[I(X)], v_{\mathrm{cx}}(I(X))\right)\right) .
$$

By Theorem 2.2.6, it suffices to consider any $I \in \mathcal{I}_{3}$, with parameters $d_{1} \in$ $\left[0, \operatorname{VaR}_{\alpha}(X)\right], d_{2} \in\left[d_{1}, \operatorname{VaR}_{\alpha}(X)\right], d_{3} \in\left[\operatorname{VaR}_{\alpha}(X)\right.$, ess $\left.\sup (X)\right]$, such that

$$
g\left(\mathbb{E}[I(X)], v_{\mathrm{cx}}(I(X))\right) \leq \pi
$$

By Intermediate Value Theorem, there exist $\tilde{d} \in[0, \operatorname{ess} \sup (X)]$ such that

(a) $\mathbb{E}\left[X-(X-\tilde{d})_{+}\right]=\mathbb{E}[I(X)]$

Define

$$
\tilde{I}(x)=x-(x-\tilde{d})_{+}, \quad \forall x \in[0, \operatorname{ess} \sup (X)]
$$

Then, $(a)$ is equivalent to that

$$
\mathbb{E}[\tilde{I}(X)]=\mathbb{E}[I(X)]
$$

The distribution function of $\tilde{I}(X)$ is given by

$$
F_{\tilde{I}(X)}(y)= \begin{cases}F_{X}(y), & \forall y \in[0, \tilde{d}), \\ 1, & \forall y \in[\tilde{d}, \operatorname{ess} \sup (X)] .\end{cases}
$$

In this case, the distribution functions $F_{\tilde{I}(X)}$ and $F_{I(X)}$ cross once with $\xi_{1}^{L}=$ $\xi_{1}^{R}=\tilde{d}$. For any $y \in\left(d_{1}, \xi_{1}^{L}\right), F_{\tilde{I}(X)}(y)<F_{I(X)}(y)$. Hence, by Theorem 2.2.3. $\tilde{I}(X) \leq_{\mathrm{cx}} I(X)$. Since $v_{c x}$ is a convex order preserving functional,

$$
v_{\mathrm{cx}}(\tilde{I}(X)) \leq v_{\mathrm{cx}}(I(X))
$$

Since $G_{E}$ and $g$ are non-decreasing functions,

$$
\begin{aligned}
& G_{E}\left(\mathbb{E}[X]-\mathbb{E}[\tilde{I}(X)]+g\left(\mathbb{E}[\tilde{I}(X)], v_{\mathrm{cx}}(\tilde{I}(X))\right)\right) \\
\leq & G_{E}\left(\mathbb{E}[X]-\mathbb{E}[I(X)]+g\left(\mathbb{E}[I(X)], v_{\mathrm{cx}}(I(X))\right)\right) .
\end{aligned}
$$


Finally, since $g$ is a non-decreasing function,

$$
g\left(\mathbb{E}[\tilde{I}(X)], v_{\mathrm{cx}}(\tilde{I}(X))\right) \leq g\left(\mathbb{E}[I(X)], v_{\mathrm{cx}}(I(X))\right) \leq \pi
$$

The above results demonstrate the powerfulness of Karlin-Novikoff-StoyanTaylor crossing conditions in Theorem 2.2.3 for solving problems of designing optimal reinsurance which involve the convex ordering. Actually, the crossing conditions can also be well applied to solve the following optimal insurance problem:

$$
\begin{cases}\inf _{I \in \mathcal{I}} & v_{c v}\left(w-T_{I}(X)\right), \\ \text { s.t. } & g\left(\mathbb{E}[I(X)], \operatorname{VaR}_{\alpha}(I(X)), \operatorname{TVaR}_{\alpha}(I(X))\right)=P,\end{cases}
$$

where $v_{\mathrm{cv}}$ is a concave order preserving functional, $w$ is the initial wealth of the insured, $g$ is a positive real-valued function on $\mathbb{R}_{+}^{3}$, and $P$ is a positive premium payment. However, since this thesis focuses on the problems of designing optimal reinsurance, reader interested in Problem 2.2.7) could refer to the results and proofs in Cheung et al. (2015a).

\subsection{Optimal Reinsurance with General Coher- ent Risk Measuring Objective and Premium}

\subsubsection{Problem Formulation}

In this section, we consider the scenario that the insurer minimizes a general coherent risk measure of her total retained loss while the premium principle determined by the reinsurer is another general coherent risk measure. Mathematically, the insurer solves:

$$
\begin{cases}\inf _{I \in \mathcal{I}} & \rho_{1}\left(T_{I}(X)\right) \\ \text { s.t. } & \rho_{2}(I(X)) \leq \pi\end{cases}
$$


where $\rho_{1}$ is a law-invariant continuous from above coherent risk measure, $\rho_{2}$ is a law-invariant comonotonic additive convex risk measure, and $\pi \in \mathbb{R}_{+}$is the budget. Note that $\rho_{2}$ is necessarily continuous from above and coherent. Solving Problem 2.3.8 consists of three essential steps.

\subsubsection{First Step: In Terms of Distortion Functions}

The first crucial step of solving Problem 2.3.8) is to express the risk measures in terms of some distortion functions. In particular, define

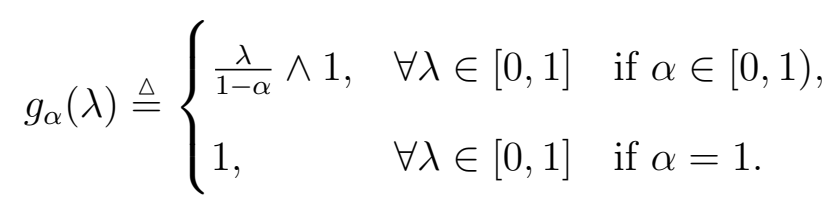

This step is composed of several representation results, which will be recalled in the following lemmas.

Before presenting the first lemma, we prove another lemma which depicts that the TVaR of a non-negative random variable can be rewritten as a distortion risk measure with the above distortion function $g_{\alpha}$, which is a standard result in the literature.

Lemma 2.3.1. Let $Y$ be a non-negative random variable. Then

$$
T \operatorname{VaR} R_{\alpha}(Y)=\int_{0}^{\operatorname{ess} \sup (Y)} g_{\alpha}\left(S_{Y}(y)\right) d y, \quad \forall \alpha \in[0,1] .
$$


Proof. It is trivial for the case with $\alpha=1$. For $\alpha \in[0,1)$, by Fubini's Theorem,

$$
\begin{aligned}
\operatorname{TVaR}_{\alpha}(Y) & =\frac{1}{1-\alpha} \int_{\alpha}^{1} \operatorname{VaR}_{\lambda}(Y) d \lambda \\
& =\frac{1}{1-\alpha} \int_{\alpha}^{1} F_{Y}^{-1}(\lambda) d \lambda \\
& =\frac{1}{1-\alpha} \int_{0}^{1-\alpha} S_{Y}^{-1}(\lambda) d \lambda \\
& =\int_{0}^{1} S_{Y}^{-1}(\lambda) d g_{\alpha}(\lambda) \\
& =\int_{0}^{1}\left(\int_{0}^{S_{Y}^{-1}(\lambda)} d y\right) d g_{\alpha}(\lambda) \\
& =\int_{0}^{\operatorname{ess} \sup (Y)}\left(\int_{0}^{S_{Y}(y)} d g_{\alpha}(\lambda)\right) d y \\
& =\int_{0}^{\operatorname{ess} \sup (Y)} g_{\alpha}\left(S_{Y}(y)\right) d y .
\end{aligned}
$$

The first lemma is a generalization of Lemma 2.3.1.

Lemma 2.3.2. Let $Y$ be a non-negative random variable. For any non-decreasing absolutely continuous function $h$ with $h(0)=0$,

$$
T \operatorname{VaR}_{\alpha}(h(Y))=\int_{0}^{\operatorname{ess} \sup (Y)} g_{\alpha}\left(S_{Y}(y)\right) d h(y), \quad \forall \alpha \in[0,1] .
$$

Proof. By Lemma 2.3.1.

$$
\begin{aligned}
\operatorname{TVaR}_{\alpha}(h(Y)) & =\int_{0}^{\operatorname{ess} \sup (Y)} g_{\alpha}\left(S_{h(Y)}(y)\right) d y \\
& =\int_{0}^{\operatorname{ess} \sup (Y)} g_{\alpha}\left(S_{Y}(y)\right) d h(y), \quad \forall \alpha \in[0,1],
\end{aligned}
$$

where the second equality is due to Equation (A.1) in Cheung and Lo (2017).

The second lemma gives a TVaR-representation for any law-invariant continuous from above coherent risk measure. 
Lemma 2.3.3. Let $Y$ be a non-negative random variable and $\rho(Y)$ be a lawinvariant continuous from above coherent risk measure of $Y$. Then

$$
\rho(Y)=\sup _{\mu \in \mathcal{M}} \int_{0}^{1} \operatorname{TVaR}_{\alpha}(Y) \mu(d \alpha),
$$

for some $\mathcal{M} \subseteq \mathcal{M}_{1}([0,1))$, where $\mathcal{M}_{1}([0,1))$ is the set of all probability measures defined on $[0,1)$.

Proof. For the proof, see Corollary 4.63 in Föllmer and Schied (2010).

The third lemma gives a TVaR-representation for any law-invariant comonotonic additive convex risk measure.

Lemma 2.3.4. Let $Y$ be a non-negative random variable and $\rho(Y)$ be a lawinvariant comonotonic additive convex risk measure of $Y$. Then

$$
\rho(Y)=\int_{0}^{1} \operatorname{TVaR}_{\alpha}(Y) \mu(d \alpha),
$$

for some $\mu \in \mathcal{M}_{1}([0,1])$, where $\mathcal{M}_{1}([0,1])$ is the set of all probability measures defined on $[0,1]$.

Proof. For the proof, see Theorem 4.93 in Föllmer and Schied (2010).

By the definition of $T_{I}(X)$ in 2.1.1) and translation invariance of $\rho_{1}$,

$$
\begin{aligned}
\rho_{1}\left(T_{I}(X)\right) & =\rho_{1}\left(X-I(X)+\rho_{2}(I(X))\right) \\
& =\rho_{1}((\operatorname{Id}-I)(X))+\rho_{2}(I(X)) .
\end{aligned}
$$

By Lemma 2.3.3, comonotonic additivity of TVaR, Lemma 2.3.2, Fubini's Theo- 
rem, and since $I \in \mathcal{I}$ is absolutely continuous with $I(0)=0$,

$$
\begin{aligned}
\rho_{1}((\mathrm{Id}-I)(X))= & \sup _{\mu_{1} \in \mathcal{M}_{1}}\left(\int_{0}^{1} \operatorname{TVaR}_{\alpha}((\operatorname{Id}-I)(X)) \mu_{1}(d \alpha)\right) \\
= & \sup _{\mu_{1} \in \mathcal{M}_{1}}\left(\int_{0}^{1} \operatorname{TVaR}_{\alpha}(X) \mu_{1}(d \alpha)-\int_{0}^{1} \operatorname{TVaR}_{\alpha}(I(X)) \mu_{1}(d \alpha)\right) \\
= & \sup _{\mu_{1} \in \mathcal{M}_{1}}\left(\int_{0}^{1} \operatorname{TVaR}_{\alpha}(X) \mu_{1}(d \alpha)\right. \\
& \left.\quad-\int_{0}^{1}\left(\int_{0}^{\operatorname{ess} \sup (X)} g_{\alpha}\left(S_{X}(x)\right) d I(x)\right) \mu_{1}(d \alpha)\right) \\
= & \sup _{\mu_{1} \in \mathcal{M}_{1}}\left(\int_{0}^{1} \operatorname{TVaR}_{\alpha}(X) \mu_{1}(d \alpha)\right. \\
& \left.-\int_{0}^{\operatorname{ess} \sup (X)}\left(\int_{0}^{1} g_{\alpha}\left(S_{X}(x)\right) \mu_{1}(d \alpha)\right) d I(x)\right),
\end{aligned}
$$

for some $\mathcal{M}_{1} \subseteq \mathcal{M}_{1}([0,1))$. Similarly, by Lemma 2.3.4, Lemma 2.3.2, Fubini's Theorem, and since $I \in \mathcal{I}$ is absolutely continuous with $I(0)=0$,

$$
\begin{aligned}
\rho_{2}(I(X)) & =\int_{0}^{1} \operatorname{TVaR}_{\alpha}(I(X)) \mu_{2}(d \alpha) \\
& =\int_{0}^{1}\left(\int_{0}^{\operatorname{ess} \sup (X)} g_{\alpha}\left(S_{X}(x)\right) d I(x)\right) \mu_{2}(d \alpha) \\
& =\int_{0}^{\operatorname{ess} \sup (X)}\left(\int_{0}^{1} g_{\alpha}\left(S_{X}(x)\right) \mu_{2}(d \alpha)\right) d I(x),
\end{aligned}
$$


for some $\mu_{2} \in \mathcal{M}_{1}([0,1])$. Therefore,

$$
\begin{aligned}
\rho_{1}\left(T_{I}(X)\right)=\sup _{\mu_{1} \in \mathcal{M}_{1}}\left(\int_{0}^{1} \operatorname{TVaR}_{\alpha}(X) \mu_{1}(d \alpha)\right. \\
\left.\quad-\int_{0}^{\operatorname{ess} \sup (X)}\left(\int_{0}^{1} g_{\alpha}\left(S_{X}(x)\right) \mu_{1}(d \alpha)\right) d I(x)\right) \\
+\int_{0}^{\operatorname{ess} \sup (X)}\left(\int_{0}^{1} g_{\alpha}\left(S_{X}(x)\right) \mu_{2}(d \alpha)\right) d I(x) \\
=\sup _{\mu_{1} \in \mathcal{M}_{1}}\left(\int_{0}^{1} \operatorname{TVaR}(X) \mu_{1}(d \alpha)\right. \\
\quad-\int_{0}^{\operatorname{ess} \sup (X)}\left(\int_{0}^{1} g_{\alpha}\left(S_{X}(x)\right) \mu_{1}(d \alpha)\right) d I(x) \\
\left.\quad+\int_{0}^{\operatorname{ess} \sup (X)}\left(\int_{0}^{1} g_{\alpha}\left(S_{X}(x)\right) \mu_{2}(d \alpha)\right) d I(x)\right) .
\end{aligned}
$$

For any $\mu_{1} \in \mathcal{M}_{1}$, define

$$
G_{1}(x) \triangleq \int_{0}^{1} g_{\alpha}\left(S_{X}(x)\right) \mu_{1}(d \alpha), \quad \forall x \in[0, \operatorname{ess} \sup (X)]
$$

Similarly, define

$$
G_{2}(x) \triangleq \int_{0}^{1} g_{\alpha}\left(S_{X}(x)\right) \mu_{2}(d \alpha), \quad \forall x \in[0, \operatorname{ess} \sup (X)]
$$

Therefore,

$$
\rho_{1}\left(T_{I}(X)\right)=\sup _{\mu_{1} \in \mathcal{M}_{1}}\left(\int_{0}^{1} \operatorname{TVaR}_{\alpha}(X) \mu_{1}(d \alpha)+\int_{0}^{\operatorname{ess} \sup (X)}\left(G_{2}(x)-G_{1}(x)\right) d I(x)\right)
$$

and

$$
\rho_{2}(I(X))=\int_{0}^{\operatorname{ess} \sup (X)} G_{2}(x) d I(x)
$$

Define

$$
\mathcal{I}(\pi) \triangleq\left\{I \in \mathcal{I}: \int_{0}^{\operatorname{ess} \sup (X)} G_{2}(x) d I(x) \leq \pi\right\} .
$$

Hence, Problem 2.3.8 is equivalent to

$$
\inf _{I \in \mathcal{I}(\pi)} \sup _{\mu_{1} \in \mathcal{M}_{1}}\left(\int_{0}^{1} \operatorname{TVaR}_{\alpha}(X) \mu_{1}(d \alpha)+\int_{0}^{\operatorname{ess} \sup (X)}\left(G_{2}(x)-G_{1}(x)\right) d I(x)\right) .
$$




\subsubsection{Second Step: Interchange of Infimum and Supre- mum}

The second crucial step of solving Problem (2.3.8), or equivalently Problem (2.3.9), is to interchange the infimum and supremum in (2.3.9). For this step, we apply one of the versions of mini-max theorem, which is called Sion's Mini-max Theorem:

Theorem 2.3.5 (Mini-max Theorem for Infinite Dimensional Space). Let $X$ and $Y$ be Hausdorff vector spaces. Let $A \subseteq X$ and $B \subseteq Y$ be non-empty compact convex subsets. Let $F$ be a real-valued function on $A \times B$ such that

(i) for each $a \in A, F(a, \cdot)$ is upper semi-continuous and quasi-concave on $B$;

(ii) for each $b \in B, F(\cdot, b)$ is lower semi-continuous and quasi-convex on $A$.

Then, $F$ satisfies the mini-max equality on $A \times B$, in the sense that

$$
\min _{a \in A} \max _{b \in B} F(a, b)=\max _{b \in B} \min _{a \in A} F(a, b) .
$$

Proof. For the proof, see Theorem 2.132 in Barbu and Precupanu (2012).

To apply Theorem 2.3.5 in 2.3.9, which involves the infimum and supremum, but not minimum and maximum, we need the following equivalences:

Proposition 2.3.6. Let $A$ and $B$ be non-empty sets. Let $F$ be a real-valued function on $A \times B$. Then the following statements are equivalent:

(i) F satisfies the mini-max equality on $A \times B$, i.e.,

$$
\min _{a \in A} \max _{b \in B} F(a, b)=\max _{b \in B} \min _{a \in A} F(a, b) ;
$$

(ii) F satisfies all of the following conditions:

(a) F has a saddle value, in the sense that

$$
\inf _{a \in A} \sup _{b \in B} F(a, b)=\sup _{b \in B} \inf _{a \in A} F(a, b) ;
$$


(b) there exists an $a^{*} \in A$ such that

$$
\inf _{a \in A} \sup _{b \in B} F(a, b)=\min _{a \in A} \sup _{b \in B} F(a, b)=\sup _{b \in B} F\left(a^{*}, b\right) ;
$$

(c) there exists an $b^{*} \in B$ such that

$$
\sup _{b \in B} \inf _{a \in A} F(a, b)=\max _{b \in B} \inf _{a \in A} F(a, b)=\inf _{a \in A} F\left(a, b^{*}\right) ;
$$

(iii) F has a saddle point, i.e., there exists a pair $\left(a^{*}, b^{*}\right) \in A \times B$ such that

$$
F\left(a^{*}, b\right) \leq F\left(a^{*}, b^{*}\right) \leq F\left(a, b^{*}\right), \quad \forall(a, b) \in A \times B .
$$

Proof. For the proof, see, for instance, Section 2.3.1 in Barbu and Precupanu (2012).

Assume, at the moment, that the conditions in Theorem 2.3.5 are satisfied. Hence, by Theorem 2.3.5 and Proposition 2.3.6. Problem (2.3.9), or equivalently Problem (2.3.8), is equivalent to

$$
\sup _{\mu_{1} \in \mathcal{M}_{1}} \inf _{I \in \mathcal{I}(\pi)}\left(\int_{0}^{1} \operatorname{TVaR}_{\alpha}(X) \mu_{1}(d \alpha)+\int_{0}^{\operatorname{ess} \sup (X)}\left(G_{2}(x)-G_{1}(x)\right) d I(x)\right),
$$

which involves solving for two optimization problems:

(i) for each $\mu_{1} \in \mathcal{M}_{1}$,

$$
V\left(\mu_{1}\right) \triangleq \inf _{I \in \mathcal{I}(\pi)}\left(\int_{0}^{1} \operatorname{TVaR}_{\alpha}(X) \mu_{1}(d \alpha)+\int_{0}^{\operatorname{ess} \sup (X)}\left(G_{2}(x)-G_{1}(x)\right) d I(x)\right) ;
$$

(ii) $\sup _{\mu_{1} \in \mathcal{M}_{1}} V\left(\mu_{1}\right)$.

Therefore, the third crucial step of solving Problem (2.3.8), or equivalently Problems 2.3.9) and 2.3.11), is to solve Problem 2.3.12). We defer the detailed examination for the validity of the conditions in Theorem 2.3.5 after solving Problem (2.3.12). 


\subsubsection{Third Step: Solving Auxiliary Optimal Reinsurance Problem}

We consider another optimal reinsurance problem when the insurer solves:

$$
\begin{cases}\inf _{I \in \mathcal{I}} & \rho_{3}\left(T_{I}(X)\right), \\ \text { s.t. } & \rho_{4}(I(X)) \leq \pi,\end{cases}
$$

where $\rho_{3}, \rho_{4}$ are law-invariant comonotonic additive convex risk measures and $\pi \in \mathbb{R}_{+}$is the budget.

By the definition of $T_{I}(X)$ in (2.1.1) and translation invariance of $\rho_{3}$,

$$
\begin{aligned}
\rho_{3}\left(T_{I}(X)\right) & =\rho_{3}\left(X-I(X)+\rho_{4}(I(X))\right) \\
& =\rho_{3}((\operatorname{Id}-I)(X))+\rho_{4}(I(X)) .
\end{aligned}
$$

By Lemma 2.3.4, comonotonic additivity of TVaR, Lemma 2.3.2, Fubini's Theorem, and since $I \in \mathcal{I}$ is absolutely continuous with $I(0)=0$,

$$
\begin{aligned}
\rho_{3}((\mathrm{Id}-I)(X))= & \int_{0}^{1} \operatorname{TVaR}_{\alpha}((\mathrm{Id}-I)(X)) \mu_{3}(d \alpha) \\
= & \int_{0}^{1} \operatorname{TVaR}_{\alpha}(X) \mu_{3}(d \alpha)-\int_{0}^{1} \operatorname{TVaR}_{\alpha}(I(X)) \mu_{3}(d \alpha) \\
= & \int_{0}^{1} \operatorname{TVaR}_{\alpha}(X) \mu_{3}(d \alpha) \\
& -\int_{0}^{1}\left(\int_{0}^{\operatorname{ess} \sup (X)} g_{\alpha}\left(S_{X}(x)\right) d I(x)\right) \mu_{3}(d \alpha) \\
= & \int_{0}^{1} \operatorname{TVaR}_{\alpha}(X) \mu_{3}(d \alpha) \\
& -\int_{0}^{\operatorname{esssup}(X)}\left(\int_{0}^{1} g_{\alpha}\left(S_{X}(x)\right) \mu_{3}(d \alpha)\right) d I(x),
\end{aligned}
$$

for some $\mu_{3} \in \mathcal{M}_{1}([0,1])$. Similarly, by Lemma 2.3.4, Lemma 2.3.2, Fubini's 
Theorem, and since $I \in \mathcal{I}$ is absolutely continuous with $I(0)=0$,

$$
\begin{aligned}
\rho_{4}(I(X)) & =\int_{0}^{1} \operatorname{TVaR}_{\alpha}(I(X)) \mu_{4}(d \alpha) \\
& =\int_{0}^{1}\left(\int_{0}^{\operatorname{ess} \sup (X)} g_{\alpha}\left(S_{X}(x)\right) d I(x)\right) \mu_{4}(d \alpha) \\
& =\int_{0}^{\operatorname{ess} \sup (X)}\left(\int_{0}^{1} g_{\alpha}\left(S_{X}(x)\right) \mu_{4}(d \alpha)\right) d I(x),
\end{aligned}
$$

for some $\mu_{4} \in \mathcal{M}_{1}([0,1])$. For $i=3,4$, define

$$
G_{i}(x) \triangleq \int_{0}^{1} g_{\alpha}\left(S_{X}(x)\right) \mu_{i}(d \alpha), \quad \forall x \in[0, \operatorname{ess} \sup (X)]
$$

Therefore,

$$
\rho_{3}\left(T_{I}(X)\right)=\int_{0}^{1} \operatorname{TVaR}_{\alpha}(X) \mu_{3}(d \alpha)+\int_{0}^{\operatorname{ess} \sup (X)}\left(G_{4}(x)-G_{3}(x)\right) d I(x)
$$

and

$$
\rho_{4}(I(X))=\int_{0}^{\operatorname{ess} \sup (X)} G_{4}(x) d I(x)
$$

Hence, Problem 2.3.13 is equivalent to

$$
\inf _{I \in \mathcal{I}(\pi)}\left(\int_{0}^{1} \operatorname{TVaR}_{\alpha}(X) \mu_{3}(d \alpha)+\int_{0}^{\operatorname{ess} \sup (X)}\left(G_{4}(x)-G_{3}(x)\right) d I(x)\right) .
$$

Therefore, solving Problem 2.3.12), for each $\mu_{1} \in \mathcal{M}_{1} \subseteq \mathcal{M}_{1}([0,1))$, is the same as solving Problem (2.3.13), if $\mu_{3}=\mu_{1}$ and $\mu_{4}=\mu_{2}$. In particular, necessarily, any $\mu_{3} \in \mathcal{M}_{1}$ does not have any point mass at $\alpha=1$. The reason that we solve Problem (2.3.12) indirectly by solving Problem 2.3.13) is that Problem 2.3.13 itself is an interesting standalone optimal reinsurance problem as well.

Thanks to a recent paper by Lo (2017), which formulates general optimal reinsurance problems from a Neyman-Pearson perspective, solving Problem 2.3.13, or equivalently Problem 2.3.14, is a direct consequence of the solution for the 
following problem:

$$
\begin{cases}\inf _{I \in \mathcal{I}} & \int_{0}^{\infty} f_{1}(x) I^{\prime}(x) d x, \\ \text { s.t. } & \int_{0}^{\infty} f_{0}(x) I^{\prime}(x) d x \leq C,\end{cases}
$$

where $f_{0}$ and $f_{1}$ are integrable functions on $\mathbb{R}_{+}$, and $C \in \mathbb{R}$. The solution of Problem 2.3.15 is given by the following theorem. Define

$$
\mathcal{G}(c) \triangleq \int_{\left\{f_{1}<c f_{0}\right\}} f_{0}(x) d x, \quad \forall c \in[-\infty, 0],
$$

and

$$
\begin{aligned}
c^{*} & \triangleq \mathcal{G}^{-1}(C) \\
& =\inf \{c \in[-\infty, 0]: \mathcal{G}(c) \geq C\} \\
& =\inf \left\{c \in[-\infty, 0]: \int_{\left\{f_{1}<c f_{0}\right\}} f_{0}(x) d x \geq C\right\} .
\end{aligned}
$$

Theorem 2.3.7 (Theorem 3.2 in Lo (2017)).

(i) If

$$
\mathcal{G}(0)\left(=\int_{\left\{f_{1}<0\right\}} f_{0}(x) d x\right) \leq C,
$$

then the optimal solutions for Problem 2.3.15) must be in the form of

$$
I_{*}^{\prime}(x)= \begin{cases}1, & \text { if } f_{1}(x)<0 \\ \gamma_{*}(x), & \text { if } f_{1}(x)=0 \\ 0, & \text { if } f_{1}(x)>0\end{cases}
$$

where $\gamma_{*}: \mathbb{R}_{+} \rightarrow[0,1]$ is any function that

$$
\int_{0}^{\infty} f_{0}(x) I_{*}^{\prime}(x) d x=\mathcal{G}(0)+\int_{\left\{f_{1}=0\right\}} f_{0}(x) \gamma_{*}(x) d x \leq C
$$

(ii) If

$$
\mathcal{G}(-\infty)\left(=\int_{\left\{f_{0}<0\right\}} f_{0}(x) d x\right) \leq C<\mathcal{G}(0)\left(=\int_{\left\{f_{1}<0\right\}} f_{0}(x) d x\right)
$$


then the optimal solutions for Problem 2.3.15) must be in the form of

$$
I_{*}^{\prime}(x)= \begin{cases}1, & \text { if } f_{1}(x)<c^{*} f_{0}(x), \\ \gamma_{*}(x), & \text { if } f_{1}(x)=c^{*} f_{0}(x), \\ 0, & \text { if } f_{1}(x)>c^{*} f_{0}(x),\end{cases}
$$

where $\gamma_{*}: \mathbb{R}_{+} \rightarrow[0,1]$ is any function that

$$
\int_{0}^{\infty} f_{0}(x) I_{*}^{\prime}(x) d x=\mathcal{G}\left(c^{*}\right)+\int_{\left\{f_{1}=c^{*} f_{0}\right\}} f_{0}(x) \gamma_{*}(x) d x=C
$$

(iii) If

$$
C<\mathcal{G}(-\infty)\left(=\int_{\left\{f_{0}<0\right\}} f_{0}(x) d x\right),
$$

then Problem (2.3.15) has no solutions.

Comparing Problems 2.3.14) and 2.3.15), we have $C=\pi$,

$$
\begin{gathered}
f_{1}(x)=G_{4}(x)-G_{3}(x), \quad \forall x \in[0, \operatorname{ess} \sup (X)], \\
f_{0}(x)=G_{4}(x), \quad \forall x \in[0, \operatorname{ess} \sup (X)], \\
\mathcal{G}(c)=\int_{\left\{(1-c) G_{4}<G_{3}\right\}} G_{4}(x) d x, \quad \forall c \in[-\infty, 0],
\end{gathered}
$$

and

$$
\begin{aligned}
c^{*} & =\mathcal{G}^{-1}(\pi) \\
& =\inf \{c \in[-\infty, 0]: \mathcal{G}(c) \geq \pi\} \\
& =\inf \left\{c \in[-\infty, 0]: \int_{\left\{(1-c) G_{4}<G_{3}\right\}} G_{4}(x) d x \geq \pi\right\} .
\end{aligned}
$$

By Theorem 2.3.7, we immediately have

\section{Theorem 2.3.8.}

(i) If

$$
\mathcal{G}(0)\left(=\int_{\left\{G_{4}<G_{3}\right\}} G_{4}(x) d x\right) \leq \pi,
$$


then the optimal ceded loss function for Problem 2.3.13) takes the form

$$
I^{*}(x)=\int_{0}^{x} \mathbb{1}_{\left\{G_{4}<G_{3}\right\}}(t) d t+\int_{0}^{x} \mathbb{1}_{\left\{G_{4}=G_{3}\right\}}(t) d \tilde{I}(t), \quad \forall x \in[0, \operatorname{ess} \sup (X)],
$$

for any $\tilde{I} \in \mathcal{I}$ such that

$$
\int_{0}^{\operatorname{ess} \sup (X)} G_{4}(x) d I^{*}(x)=\int_{\left\{G_{4}<G_{3}\right\}} G_{4}(x) d x+\int_{\left\{G_{4}=G_{3}\right\}} G_{4}(x) d \tilde{I}(x) \leq \pi ;
$$

(ii) If

$$
\mathcal{G}(-\infty)(=0) \leq \pi<\mathcal{G}(0)\left(=\int_{\left\{G_{4}<G_{3}\right\}} G_{4}(x) d x\right),
$$

then the optimal ceded loss function for Problem (2.3.13) takes the form

$$
\begin{array}{r}
I^{*}(x)=\int_{0}^{x} \mathbb{1}_{\left\{\left(1-c^{*}\right) G_{4}<G_{3}\right\}}(t) d t+\int_{0}^{x} \mathbb{1}_{\left\{\left(1-c^{*}\right) G_{4}=G_{3}\right\}}(t) d \tilde{I}(t), \\
\forall x \in[0, \operatorname{ess} \sup (X)],
\end{array}
$$

for any $\tilde{I} \in \mathcal{I}$ such that

$$
\begin{aligned}
\int_{0}^{\operatorname{ess} \sup (X)} G_{4}(x) d I^{*}(x) & =\int_{\left\{\left(1-c^{*}\right) G_{4}<G_{3}\right\}} G_{4}(x) d x+\int_{\left\{\left(1-c^{*}\right) G_{4}=G_{3}\right\}} G_{4}(x) d \tilde{I}(x) \\
& =\pi .
\end{aligned}
$$

Moreover, the optimal objective value (2.3.13) is given by

$$
c^{*} \pi+\int_{0}^{\operatorname{ess} \sup (X)} \min \left\{G_{3}(x),\left(1-c^{*}\right) G_{4}(x)\right\} d x .
$$

Proof. Only the optimal objective value needs to be proved, since the optimal ceded loss functions in both cases are direct consequences of Theorem 2.3.7.

For case (i), by Lemma 2.3.2 and Fubini's Theorem, the optimal objective 
value 2.3 .13 is given by

$$
\begin{aligned}
& \inf _{I \in \mathcal{I}(\pi)}\left(\int_{0}^{1} \operatorname{TVaR}_{\alpha}(X) \mu_{3}(d \alpha)+\int_{0}^{\operatorname{ess} \sup (X)}\left(G_{4}(x)-G_{3}(x)\right) d I(x)\right) \\
= & \int_{0}^{1}\left(\int_{0}^{\operatorname{ess} \sup (X)} g_{\alpha}\left(S_{X}(x)\right) d x\right) \mu_{3}(d \alpha)+\int_{0}^{\operatorname{ess} \sup (X)}\left(G_{4}(x)-G_{3}(x)\right) d I^{*}(x) \\
= & \int_{0}^{\operatorname{ess} \sup (X)}\left(\int_{0}^{1} g_{\alpha}\left(S_{X}(x)\right) \mu_{3}(d \alpha)\right) d x+\int_{\left\{G_{4}<G_{3}\right\}}\left(G_{4}(x)-G_{3}(x)\right) d x \\
= & \int_{0}^{\operatorname{ess} \sup (X)} G_{3}(x) d x+\int_{\left\{G_{4}<G_{3}\right\}}\left(G_{4}(x)-G_{3}(x)\right) d x \\
= & \int_{\left\{G_{4} \geq G_{3}\right\}}^{\operatorname{ess} \sup (X)} \min \left\{G_{3}(x), G_{4}(x)\right\} d x \\
= & \int_{0}^{\operatorname{ess} \sup (X)} \min \left\{G_{4}(x),\left(1-c^{*}\right) G_{4}(x)\right\} d x, \\
= & c^{*} \pi+\int_{0}^{\operatorname{man}(x)} \min (x)
\end{aligned}
$$

where the last equality holds since $c^{*}=0$ for case (i).

Similarly, for case (ii), by Lemma 2.3.2, Fubini's Theorem, and the budget 
constraint (2.3.16), the optimal objective value 2.3 .13 is given by

$$
\begin{aligned}
& \inf _{I \in \mathcal{I}(\pi)}\left(\int_{0}^{1} \operatorname{TVaR}_{\alpha}(X) \mu_{3}(d \alpha)+\int_{0}^{\operatorname{ess} \sup (X)}\left(G_{4}(x)-G_{3}(x)\right) d I(x)\right) \\
= & \int_{0}^{\operatorname{ess} \sup (X)} G_{3}(x) d x+\int_{0}^{\operatorname{ess} \sup (X)}\left(G_{4}(x)-G_{3}(x)\right) d I^{*}(x) \\
= & \int_{0}^{\operatorname{ess} \sup (X)} G_{3}(x) d x+\int_{\left\{\left(1-c^{*}\right) G_{4}<G_{3}\right\}}\left(G_{4}(x)-G_{3}(x)\right) d x \\
& +\int_{\left\{\left(1-c^{*}\right) G_{4}=G_{3}\right\}}\left(G_{4}(x)-G_{3}(x)\right) d \tilde{I}(x) \\
= & \int_{\left\{\left(1-c^{*}\right) G_{4} \geq G_{3}\right\}} G_{3}(x) d x+\int_{\left\{\left(1-c^{*}\right) G_{4}<G_{3}\right\}} G_{4}(x) d x+c^{*} \int_{\left\{\left(1-c^{*}\right) G_{4}=G_{3}\right\}} G_{4}(x) d \tilde{I}(x) \\
= & \int_{\left\{\left(1-c^{*}\right) G_{4} \geq G_{3}\right\}} G_{3}(x) d x+\int_{\left\{\left(1-c^{*}\right) G_{4}<G_{3}\right\}} G_{4}(x) d x+c^{*} \pi-c^{*} \int_{\left\{\left(1-c^{*}\right) G_{4}<G_{3}\right\}} G_{4}(x) d x \\
= & c^{*} \pi+\int_{\left\{\left(1-c^{*}\right) G_{4} \geq G_{3}\right\}}^{\operatorname{ess} \sup (X)} G_{3}(x) d x+\int_{\left\{\left(1-c^{*}\right) G_{4}<G_{3}\right\}}\left(1-c^{*}\right) G_{4}(x) d x \\
= & c^{*} \pi+\int_{0}^{\min \left\{G_{3}(x),\left(1-c^{*}\right) G_{4}(x)\right\} d x .}
\end{aligned}
$$

Therefore, for each $\mu_{1} \in \mathcal{M}_{1}$ which does not have any point mass at $\alpha=1$, the optimal ceded loss function and the optimal objective value for Problem (2.3.12) is given by Theorem 2.3.8, with replacing $G_{3}$ and $G_{4}$ by $G_{1}$ and $G_{2}$ respectively. Hence, these solve Problem (2.3.11), or equivalently Problems (2.3.8) and 2.3.9). However, before presenting the solution for Problem (2.3.8), we need to verify the conditions in Theorem 2.3.5. In the remaining sections, we assume that the random loss $X$ is continuous and bounded. 


\subsubsection{Second Step Continued: Conditions for Mini-max Theorem}

Let $\mathrm{BV}[0, \operatorname{ess} \sup (X)]$ be the set of all real-valued functions $I$ on $[0, \operatorname{ess} \sup (X)]$, normalized so that $I(0)=0$ and is right continuous, with bounded variation, i.e.,

$$
\|I\|_{\mathrm{BV}} \triangleq \sup _{n \in \mathbb{N} ; 0=x_{0} \leq x_{1} \leq \cdots \leq x_{n} \leq \operatorname{ess} \sup (X)} \sum_{i=1}^{n}\left|I\left(x_{i}\right)-I\left(x_{i-1}\right)\right|<\infty .
$$

Let $B_{\mathrm{BV}}(\operatorname{ess} \sup (X))$ be the closed ball

$$
B_{\mathrm{BV}}(\operatorname{ess} \sup (X)) \triangleq\left\{I \in \mathrm{BV}[0, \operatorname{ess} \sup (X)]:\|I\|_{\mathrm{BV}} \leq \operatorname{ess} \sup (X)\right\}
$$

in $\operatorname{BV}[0, \operatorname{ess} \sup (X)]$. Then, we have

$$
\mathcal{I} \subseteq B_{\mathrm{BV}}(\operatorname{ess} \sup (X))
$$

Indeed, for $I \in \mathcal{I}$, since $I$ is non-decreasing and $I(0)=0$,

$$
\begin{aligned}
\|I\|_{\mathrm{BV}} & =\sup _{n \in \mathbb{N} ; 0=x_{0} \leq x_{1} \leq \cdots \leq x_{n} \leq \operatorname{ess} \sup (X)} \sum_{i=1}^{n}\left|I\left(x_{i}\right)-I\left(x_{i-1}\right)\right| \\
& =\sup _{n \in \mathbb{N} ; 0=x_{0} \leq x_{1} \leq \cdots \leq x_{n} \leq \operatorname{ess} \sup (X)} \sum_{i=1}^{n}\left(I\left(x_{i}\right)-I\left(x_{i-1}\right)\right) \\
& =\sup _{n \in \mathbb{N} ; 0=x_{0} \leq x_{1} \leq \cdots \leq x_{n} \leq \operatorname{ess} \sup (X)} I\left(x_{n}\right) \\
& =I(\operatorname{ess} \sup (X)) \\
& \leq \operatorname{ess} \sup (X) \\
& <\infty,
\end{aligned}
$$

since $X$ is bounded. Therefore, $I \in B_{\mathrm{BV}}(\operatorname{ess} \sup (X))$. Hence,

$$
\mathcal{I} \subseteq \operatorname{BV}[0, \operatorname{ess} \sup (X)]
$$

It is well-known that (i) $\mathrm{BV}[0, \operatorname{ess} \sup (X)]$ is a vector space endowed with the usual addition and scalar multiplication operations for real-valued functions; and 
(ii) $\|\cdot\|_{\mathrm{BV}}$ is a norm on $\mathrm{BV}[0, \operatorname{ess} \sup (X)]$. Hence, $\left(\mathrm{BV}[0, \operatorname{ess} \sup (X)],\|\cdot\|_{\mathrm{BV}}\right)$ is a normed vector space, and thus is a topological vector space, using the open balls as the base for the (strong) topology. Moreover, $\left(\mathrm{BV}[0, \operatorname{ess} \sup (X)],\|\cdot\|_{\mathrm{BV}}\right)$ is Banach and Hausdorff space.

Let $C[0, \operatorname{ess} \sup (X)]$ be the set of all real-valued continuous functions on $[0, \operatorname{ess} \sup (X)]$. It is well-known that $C[0, \operatorname{ess} \sup (X)]$ is a vector space endowed with the usual addition and scalar multiplication operations for real-valued functions. Hence, $\left(C[0, \operatorname{ess} \sup (X)],\|\cdot\|_{\infty}\right)$ is a normed vector space, and thus is a topological vector space, using the open balls as the base for the topology. Moreover, $\left(C[0, \operatorname{ess} \sup (X)],\|\cdot\|_{\infty}\right)$ is separable, Banach and Hausdorff space.

Define the weak*-topology $\tau$ on the topological dual space $C[0, \operatorname{ess} \sup (X)]^{*}$ of $C[0, \operatorname{ess} \sup (X)]$, which is the space of all continuous linear functionals on $C[0, \operatorname{ess} \sup (X)]$. Hence, for any $\phi \in C[0, \operatorname{ess} \sup (X)]$, the map

$$
T_{\phi}(\psi)=\psi(\phi), \quad \forall \psi \in C[0, \operatorname{ess} \sup (X)]^{*}
$$

is continuous with respect to the weak*-topology $\tau$.

By Riesz representation theorem, for any $\psi \in C[0, \operatorname{ess} \sup (X)]^{*}$, there exists a unique $I \in \mathrm{BV}[0, \operatorname{ess} \sup (X)]$ such that

$$
\psi(\phi)=\int_{0}^{\operatorname{ess} \sup (X)} \phi(x) d I(x), \quad \forall \phi \in C[0, \operatorname{ess} \sup (X)] .
$$

Conversely, for any $I \in \mathrm{BV}[0$, ess $\sup (X)]$, we could define a linear functional on $C[0, \operatorname{ess} \sup (X)]$ as above. Hence, the topological dual space $C[0, \operatorname{ess} \sup (X)]^{*}$ of $C[0, \operatorname{ess} \sup (X)]$, can be identified as $\operatorname{BV}[0, \operatorname{ess} \sup (X)]$.

Therefore, the weak*-topology $\tau$ is defined on $\operatorname{BV}[0, \operatorname{ess} \sup (X)]$ such that, for any $\phi \in C[0, \operatorname{ess} \sup (X)]$, the map

$$
T_{\phi}(I)=\int_{0}^{\operatorname{ess} \sup (X)} \phi(x) d I(x), \quad \forall I \in \operatorname{BV}[0, \operatorname{ess} \sup (X)]
$$


is continuous with respect to the weak ${ }^{*}$-topology $\tau$. Moreover, since $\left(C[0, \operatorname{ess} \sup (X)],\|\cdot\|_{\infty}\right)$ is a normed vector space, $(\mathrm{BV}[0, \operatorname{ess} \sup (X)], \tau)$ is Hausdorff.

We want to show that $(\mathcal{I}, \tau)$ is a compact topological space. By BanachAlaoglu theorem, the closed ball $B_{\mathrm{BV}}(\operatorname{ess} \sup (X))$ is compact in the weak*-topology $\tau$. Since

$$
\mathcal{I} \subseteq B_{\mathrm{BV}}(\operatorname{ess} \sup (X))
$$

it suffices to show that $\mathcal{I}$ is closed in the weak ${ }^{*}$-topology $\tau$. Since $\left(C[0, \operatorname{ess} \sup (X)],\|\cdot\|_{\infty}\right)$ is separable, by Proposition 3.24 in Fabian et al. (2001), $\left(B_{\mathrm{BV}}(\operatorname{ess} \sup (X)), \tau\right)$ is metrizable. Hence, it suffices to show that $\mathcal{I}$ is sequentially closed in the weak*topology $\tau$.

Let $\left\{I_{n}\right\}$ be a sequence in $\mathcal{I}$ such that $I_{n} \stackrel{*}{\rightarrow} I$ for some $I \in B_{\mathrm{BV}}(\operatorname{ess} \sup (X))$, i.e., for any $\phi \in C[0, \operatorname{ess} \sup (X)]$,

$$
\int_{0}^{\operatorname{ess} \sup (X)} \phi(x) d I_{n}(x) \rightarrow \int_{0}^{\operatorname{ess} \sup (X)} \phi(x) d I(x) .
$$

Since $I \in B_{\mathrm{BV}}(\operatorname{ess} \sup (X)) \subseteq \mathrm{BV}[0, \operatorname{ess} \sup (X)], I(0)=0$. Since $I_{n} \in \mathcal{I}$, implying that $I_{n}$ is increasing on $[0, \operatorname{ess} \sup (X)], I$ is also increasing on $[0, \operatorname{ess} \sup (X)]$. Indeed, if there exist $a, b \in[0, \operatorname{ess} \sup (X)]$ such that $a<b$ but $I(a)>I(b)$, then one can choose a $\phi \in C[0, \operatorname{ess} \sup (X)]$ such that $\phi(x) \geq 0$ for any $x \in[a, b]$ while $\phi(x)=0$ for $x \notin[a, b]$ while

$$
\int_{0}^{\operatorname{ess} \sup (X)} \phi(x) d I(x)<0 .
$$

However, for any $n \in \mathbb{N}$,

$$
\int_{0}^{\operatorname{ess} \sup (X)} \phi(x) d I_{n}(x) \geq 0
$$

implying that

$$
\int_{0}^{\operatorname{ess} \sup (X)} \phi(x) d I(x) \geq 0
$$


which leads to contradiction. For instance, define

$$
\phi(x)= \begin{cases}C^{2}(x-a) & \text { for } x \in\left[a, a+\frac{1}{C}\right], \\ C & \text { for } x \in\left[a+\frac{1}{C}, b-\frac{1}{C}\right], \\ C^{2}(b-x) & \text { for } x \in\left[b-\frac{1}{C}, b\right], \\ 0 & \text { for } x \notin[a, b]\end{cases}
$$

for some constant $C>\frac{2}{b-a}$. Obviously, $\phi(x) \geq 0$ for any $x \in[a, b]$ while $\phi(x)=0$ for $x \notin[a, b]$. We can choose a large enough $C>\frac{2}{b-a}$ such that

$$
\int_{0}^{\operatorname{ess} \sup (X)} \phi(x) d I(x) \approx \int_{a}^{b} C d I(x)=C(I(b)-I(a))<0 .
$$

Therefore, $I$ is increasing on $[0, \operatorname{ess} \sup (X)]$. By Portemanteau's theorem, see, for instance, Theorem 13.16 in Klenke (2014) or Theorem 2.1 in Billingsley (2009), since $I_{n} \stackrel{*}{\rightarrow} I$,

$$
I_{n}(x) \rightarrow I(x)
$$

for all $x \in[0, \operatorname{ess} \sup (X)]$ such that $I(x)$ is continuous. Since $I$ is increasing on $[0, \operatorname{ess} \sup (X)], I$ is continuous on $[0, \operatorname{ess} \sup (X)]$ except countably many points on $[0, \operatorname{ess} \sup (X)]$, say except on $E \subseteq[0, \operatorname{ess} \sup (X)]$. Then, $I$ is continuous on $D=[0, \operatorname{ess} \sup (X)] \backslash E$, which is dense in $[0, \operatorname{ess} \sup (X)]$. For any $x \in D$,

$$
\lim _{n \rightarrow \infty} I_{n}(x)=I(x)
$$

Let $x \in E$. By denseness, there exist $\left\{x_{m}\right\} \subseteq D$ such that $x_{m} \downarrow x$. Since $I_{n} \in \mathcal{I}$,

$$
0 \leq I_{n}\left(x_{m}\right)-I_{n}(x) \leq x_{m}-x
$$

Take $n \rightarrow \infty$, we have

$$
0 \leq I\left(x_{m}\right)-\lim _{n \rightarrow \infty} I_{n}(x) \leq x_{m}-x .
$$

Then, take $m \rightarrow \infty$, we have

$$
\lim _{n \rightarrow \infty} I_{n}(x)=\lim _{m \rightarrow \infty} I\left(x_{m}\right)
$$


Since $I \in \mathrm{BV}[0, \operatorname{ess} \sup (X)], I$ is right-continuous on $[0, \operatorname{ess} \sup (X)]$. Therefore,

$$
\lim _{m \rightarrow \infty} I\left(x_{m}\right)=I(x)
$$

So, for any $x \in[0, \operatorname{ess} \sup (X)]$,

$$
I_{n}(x) \rightarrow I(x)
$$

and hence, for any $x, y \in[0, \operatorname{ess} \sup (X)]$ with $y<x$,

$$
0 \leq I_{n}(x)-I_{n}(y) \leq x-y
$$

implies that

$$
0 \leq I(x)-I(y) \leq x-y
$$

Therefore, $I \in \mathcal{I}$, and thus $\mathcal{I}$ is sequentially closed in the weak ${ }^{*}$-topology $\tau$. Hence $(\mathcal{I}, \tau)$ is a compact topological space. By the continuity of $G_{2}$, it is clear that $\mathcal{I}(\pi)$ is a closed subset of $\mathcal{I}$. Hence, $\mathcal{I}(\pi)$ is a compact subset of the Hausdorff vector space $\mathrm{BV}[0, \operatorname{ess} \sup (X)]$ in the weak*-topology $\tau$.

Let $\mathcal{M}^{S}([0,1))$ be the set of all finite signed measures $\mu$ on $[0,1)$. Define

$$
\|\mu\|_{\mathrm{TV}}=|\mu|([0,1)), \quad \forall \mu \in \mathcal{M}^{S}([0,1)) .
$$

It is well-known that (i) $\mathcal{M}^{S}([0,1))$ is a vector space endowed with the usual addition and scalar multiplication operations for signed measures; and (ii) $\|\cdot\|_{\mathrm{TV}}$ is a norm on $\mathcal{M}^{S}([0,1))$. Hence, $\left(\mathcal{M}^{S}([0,1)),\|\cdot\|_{\mathrm{TV}}\right)$ is a normed vector space, and thus is a topological vector space, using the open balls as the base for the (strong) topology. Moreover, $\left(\mathcal{M}^{S}([0,1)),\|\cdot\|_{\mathrm{TV}}\right)$ is Banach and Hausdorff space.

By the similar arguments as above and the one-to-one correspondence between signed measures and right-continuous functions of bounded variation, the weak*topology $\tilde{\tau}$ is defined on $\mathcal{M}^{S}([0,1))$ such that, for any $\phi \in C[0,1)$, the map

$$
\tilde{T}_{\phi}(\mu)=\int_{0}^{1} \phi(\alpha) \mu(d \alpha), \quad \forall \mu \in \mathcal{M}^{S}([0,1))
$$


is continuous with respect to the weak*-topology $\tilde{\tau}$. Moreover, since $\left(C[0,1),\|\cdot\|_{\infty}\right)$ is a normed vector space, $\left(\mathcal{M}^{S}([0,1)), \tau\right)$ is Hausdorff.

To sum up, firstly, we showed that $\mathcal{I}(\pi)$ is a compact convex subset of the Hausdorff vector space BV $[0$, ess $\sup (X)]$ in the weak*-topology $\tau$. Moreover, for each $\mu_{1} \in \mathcal{M}_{1}$, since $X$ is continuous on $[0, \operatorname{ess} \sup (X)], G_{2}-G_{1}$ is continuous on $[0, \operatorname{ess} \sup (X)]$, i.e., $G_{2}-G_{1} \in C[0, \operatorname{ess} \sup (X)]$, and thus

$V\left(I, \mu_{1}\right)=\int_{0}^{1} \operatorname{TVaR}_{\alpha}(X) \mu_{1}(d \alpha)+\int_{0}^{\operatorname{ess} \sup (X)}\left(G_{2}(x)-G_{1}(x)\right) d I(x), \quad \forall I \in \mathcal{I}(\pi)$, is continuous in $\mathcal{I}(\pi)$ with respect to the weak ${ }^{*}$-topology $\tau$. It is also obvious that $V\left(\cdot, \mu_{1}\right)$ is linear in $\mathcal{I}(\pi)$.

Secondly, we showed that $\mathcal{M}_{1}$ is a subset of the Hausdorff vector space $\mathcal{M}^{S}([0,1))$ in the weak*-topology $\tilde{\tau}$. We shall assume that $\mathcal{M}_{1}$ is convex and compact with respect to the weak ${ }^{*}$-topology $\tilde{\tau}$. Moreover, for each $I \in \mathcal{I}(\pi)$, since TVaR. $((\operatorname{Id}-I)(X))$ is continuous on $[0,1)$, i.e., $\mathrm{TVaR} .((\mathrm{Id}-I)(X)) \in C[0,1)$, $V\left(I, \mu_{1}\right)=\int_{0}^{1} \operatorname{TVaR}_{\alpha}((\operatorname{Id}-I)(X)) \mu_{1}(d \alpha)+\int_{0}^{1} \operatorname{TVaR}_{\alpha}(I(X)) \mu_{2}(d \alpha), \quad \forall \mu_{1} \in \mathcal{M}_{1}$, is continuous in $\mathcal{M}_{1}$ with respect to the weak*-topology $\tilde{\tau}$. It is also obvious that $V(I, \cdot)$ is linear in $\mathcal{M}_{1}$.

These verify the conditions in Theorem 2.3.5.

\subsubsection{Solution of Problem (2.3.8)}

For any $\mu_{1} \in \mathcal{M}_{1}$, define

$$
\mathcal{G}\left(\mu_{1}, c\right) \triangleq \int_{\left\{(1-c) G_{2}<G_{1}\right\}} G_{2}(x) d x, \quad \forall c \in[-\infty, 0],
$$

and

$$
\begin{aligned}
c^{*}\left(\mu_{1}\right) & \triangleq \inf \left\{c \in[-\infty, 0] \mid \mathcal{G}\left(\mu_{1}, c\right) \geq \pi\right\} \\
& =\inf \left\{c \in[-\infty, 0] \mid \int_{\left\{(1-c) G_{2}<G_{1}\right\}} G_{2}(x) d x \geq \pi\right\} .
\end{aligned}
$$


Hence, by Theorem 2.3.8 and the verification of the conditions for Theorem 2.3.5 in Section 2.3.5, the solution for Problem 2.3.8), or equivalently Problems 2.3.9) and (2.3.11), is given by the following theorem.

Theorem 2.3.9. Assume that the random loss $X$ is continuous and bounded and $\mathcal{M}_{1}$ is convex and compact with respect to the weak ${ }^{*}$-topology $\tilde{\tau}$, as defined in Section 2.3.5. Denote $\mu_{1}^{*} \in \mathcal{M}_{1}$ as the maximizer of the problem

$$
\max _{\mu_{1} \in \mathcal{M}_{1}}\left(c^{*}\left(\mu_{1}\right) \pi+\int_{0}^{\operatorname{ess} \sup (X)} \min \left\{G_{1}(x),\left(1-c^{*}\left(\mu_{1}\right)\right) G_{2}(x)\right\} d x\right) .
$$

Then,

(i) if

$$
\mathcal{G}\left(\mu_{1}^{*}, 0\right)\left(=\int_{\left\{G_{2}<G_{1}^{*}\right\}} G_{2}(x) d x\right) \leq \pi,
$$

then the optimal ceded loss function for Problem (2.3.8) takes the form

$$
I^{*}(x)=\int_{0}^{x} \mathbb{1}_{\left\{G_{2}<G_{1}^{*}\right\}}(t) d t+\int_{0}^{x} \mathbb{1}_{\left\{G_{2}=G_{1}^{*}\right\}}(t) d \tilde{I}(t), \quad \forall x \in[0, \operatorname{ess} \sup (X)],
$$

for any $\tilde{I} \in \mathcal{I}$ such that

$$
\int_{0}^{\operatorname{ess} \sup (X)} G_{2}(x) d I^{*}(x)=\int_{\left\{G_{2}<G_{1}^{*}\right\}} G_{2}(x) d x+\int_{\left\{G_{2}=G_{1}^{*}\right\}} G_{2}(x) d \tilde{I}(x) \leq \pi
$$

(ii) if

$$
\mathcal{G}\left(\mu_{1}^{*},-\infty\right)(=0) \leq \pi<\mathcal{G}\left(\mu_{1}^{*}, 0\right)\left(=\int_{\left\{G_{2}<G_{1}^{*}\right\}} G_{2}(x) d x\right),
$$

then the optimal ceded loss function for Problem (2.3.8) takes the form

$$
\begin{array}{r}
I^{*}(x)=\int_{0}^{x} \mathbb{1}_{\left\{\left(1-c^{*}\left(\mu_{1}^{*}\right)\right) G_{2}<G_{1}^{*}\right\}}(t) d t+\int_{0}^{x} \mathbb{1}_{\left\{\left(1-c^{*}\left(\mu_{1}^{*}\right)\right) G_{2}=G_{1}^{*}\right\}}(t) d \tilde{I}(t), \\
\forall x \in[0, \operatorname{ess} \sup (X)],
\end{array}
$$

for any $\tilde{I} \in \mathcal{I}$ such that

$$
\begin{aligned}
\int_{0}^{\operatorname{ess} \sup (X)} G_{2}(x) d I^{*}(x)= & \int_{\left\{\left(1-c^{*}\left(\mu_{1}^{*}\right)\right) G_{2}<G_{1}^{*}\right\}} G_{2}(x) d x \\
& +\int_{\left\{\left(1-c^{*}\left(\mu_{1}^{*}\right)\right) G_{2}=G_{1}^{*}\right\}} G_{2}(x) d \tilde{I}(x) \\
= & \pi .
\end{aligned}
$$




\section{Chapter 3}

\section{Forward Entropic Risk Measures}

\subsection{Motivation of Forward Entropic Risk Mea- sures: Maturity-independent Risk Measure- ment}

In the first section of this chapter, we motivate the study of the forward entropic risk measures, which were first introduced in Zariphopoulou and Žitković (2010). Let $W=\left\{W_{t}\right\}_{t \geq 0}$ be a $d$-dimensional Brownian motion on a probability space $(\Omega, \mathcal{F}, \mathbb{P})$. Denote by $\mathbb{F}=\left\{\mathcal{F}_{t}\right\}_{t \geq 0}$ the augmented filtration generated by $W$. Assume that we have a financial market environment building upon this filtered probability space. We first recall how dynamic risk measures on the risk positions in the financial market are defined.

At the initial time $t=0$, fix any terminal date $T>0$, and thus, the investment horizon $[0, T]$ is fixed. Denote $\mathcal{L}^{\infty}\left(\mathcal{F}_{T}\right)$ as the space of all $\mathcal{F}_{T}$-measurable and uniformly bounded random variable defined on the filtered probability space. The conditional risk measure $\rho_{t, T}$, at any intermediary time $t \in[0, T]$, is defined as a mapping from $\mathcal{L}^{\infty}\left(\mathcal{F}_{T}\right)$ to $\mathcal{L}^{\infty}\left(\mathcal{F}_{t}\right)$, the space of all $\mathcal{F}_{t}$-measurable and uniformly 
bounded random variable, which satisfies certain reasonable axioms, namely, conditional translation invariance and monotonicity. The dynamic risk measure $\rho_{\cdot, T}$ is then defined as the family of conditional risk measures $\rho_{\cdot, T}=\left\{\rho_{t, T}\right\}_{t \in[0, T]}$.

From the definition of dynamic risk measures, we observe an implicit but crucial assumption being made at the initial time $t=0$. It is assumed that all risk positions in consideration will be introduced and matured at any time in the fixed investment horizon $[0, T]$. However, this is not a realistic assumption, and hence, the dynamic risk measure, solely defined in the fixed investment horizon $[0, T]$, fails to manage any new risk positions with larger maturities. Mathematically, although we may have complete knowledge of all risk positions maturing in the fixed investment horizon $[0, T]$ at the initial time $t=0$, a new risk position, with the maturity $T^{\prime}>T$, may be introduced at a post-initial time $s \in(0, T)$. The dynamic risk measure $\rho_{\cdot, T}=\left\{\rho_{t, T}\right\}_{t \in[0, T]}$, defined at the initial time $t=0$, cannot evaluate the new risk position since the conditional risk measures are not defined for times $t \in\left(T, T^{\prime}\right]$. At time $s \in(0, T)$, the simplest way to remedy this situation is to extend the dynamic risk measure $\rho_{\cdot, T}=\left\{\rho_{t, T}\right\}_{t \in[0, T]}$ to a new dynamic risk measure $\tilde{\rho}_{\cdot, T^{\prime}}=\left\{\tilde{\rho}_{t, T^{\prime}}\right\}_{t \in\left[s, T^{\prime}\right]}$. If the intrinsic time consistency is essential, $\rho_{\cdot, T}$ and $\tilde{\rho}_{\cdot, T^{\prime}}$ must be related by

$$
\tilde{\rho}_{t, T^{\prime}}=\rho_{t, T} \quad \forall t \in[s, T] .
$$

On the other hand, if the intrinsic time consistency needs to be preserved, we must define the new dynamic risk measure $\tilde{\rho}_{\cdot, T^{\prime}}$, at time $s \in(0, T)$, using the market model at the initial time $t=0$. Hence, any new information of changing market conditions within the time period $(0, s)$ and modification of the market model cannot be incorporated into the new dynamic risk measure $\tilde{\rho}_{\cdot, T^{\prime}}$. In addition, it is not clear how to reasonably define the conditional risk measures $\tilde{\rho}_{t, T^{\prime}}$, for times $t \in\left(T, T^{\prime}\right]$.

However, in an arbitrage-free valuation setting, we do not encounter such 
issues. The linear pricing operator, which is simply the conditional expectation of the risk positions, is defined for all times and is independent of the maturity of each risk position in consideration. The pricing operator works well without the need to know a priori what kind of risk positions will be introduced and when will the risk positions mature. Therefore, maturity independent risk measures should be introduced, which are non-linear risk measurements preserving the property of maturity independence in the linear pricing operator, to remedy the above issues in the dynamic risk measures.

For the sake of completeness, we recall the definition of the maturity independent (convex) risk measures. Define the space of all uniformly bounded risk positions by

$$
\mathcal{L} \triangleq \cup_{t \geq 0} \mathcal{L}^{\infty}\left(\mathcal{F}_{t}\right)
$$

Definition 3.1.1. For any $\xi \in \mathcal{L}$, define

$$
T_{\xi} \triangleq \inf \left\{T \geq 0: \xi \in \mathcal{F}_{T}\right\}
$$

The maturity independent convex risk measure $\rho$ on $\mathcal{L}$ is a family of risk measures $\rho=\left\{\rho_{t}\right\}_{t \in[0, T .]}$, such that, for any $\xi \in \mathcal{L}$ and for each $t \in\left[0, T_{\xi}\right], \rho_{t}$ satisfies all of the following properties:

(i) (Anti-positivity) if $\xi \geq 0$ a.s., $\rho_{t}(\xi) \leq 0$ a.s.;

(ii) (Translation invariance) for any $m \in \mathcal{L}^{\infty}\left(\mathcal{F}_{t}\right), \rho_{t}(\xi-m)=\rho_{t}(\xi)+m$;

(iii) (Convexity) for any constant $\alpha \in[0,1]$ and $\xi^{\prime} \in \mathcal{L}$, if $t \in\left[0, T_{\xi} \wedge T_{\xi^{\prime}}\right]$, $\rho_{t}\left(\alpha \xi+(1-\alpha) \xi^{\prime}\right) \leq \alpha \rho_{t}(\xi)+(1-\alpha) \rho_{t}\left(\xi^{\prime}\right)$

(iv) (Time consistency and maturity independence) for any $s \in\left[t, T_{\xi}\right], \rho_{t}(\xi)=$ $\rho_{t}\left(-\rho_{s}(\xi)\right)$.

We shall show that the forward entropic risk measures, the main subject of this chapter, are maturity independent convex risk measures, in particular, satisfying the property of time consistency and maturity independence. Moreover, 
the forward entropic risk measures, similar to the classical entropic risk measures, are defined in a decision theoretic framework. Together with the fact that the maturity independence risk measures remedy the deficiency in the dynamic risk measures, this motivates us to further study the forward entropic risk measures. The next section devotes to recall the definition of the forward entropic risk measures.

\subsection{Forward Entropic Risk Measures}

Assume that we have a financial market environment building upon the filtered probability space $\left(\Omega, \mathcal{F}, \mathbb{F}=\left\{\mathcal{F}_{t}\right\}_{t \geq 0}, \mathbb{P}\right)$. Let $\pi$ be an investment strategy defined for all times $t \geq 0$, and denote $X^{\pi}=\left\{X_{t}^{\pi}\right\}_{t \geq 0}$ as the corresponding wealth process. We shall use the superscript $t, x$, whenever appropriate, to indicate the dependence on the initial condition $x \in \mathbb{R}$ of the wealth process at the initial time $t \geq 0$. Denote $\mathcal{A}$ as the set of admissible investment strategies defined for all times $t \geq 0$.

To define the forward entropic risk measures, we first define the (exponential) forward investment performance processes, which were developed by Musiela and Zariphopoulou (2005, 2007, 2008, 2009a,b, 2010a, b) as the complements of the classical expected utility theory:

Definition 3.2.1. A process $\{U(\omega, x, t)\}_{\omega \in \Omega, x \in \mathbb{R}, t \geq 0}$ is called a forward investment performance process if it satisfies all of the following properties:

(i) for each $x \in \mathbb{R},\{U(\omega, x, t)\}_{\omega \in \Omega, t \geq 0}$ is $\mathbb{F}$-progressively measurable;

(ii) for each $\omega \in \Omega$ and $t \geq 0,\{U(\omega, x, t)\}_{x \in \mathbb{R}}$ is strictly increasing and strictly concave;

(iii) for any $\pi \in \mathcal{A}$,

$$
\mathbb{E}\left[U\left(X_{s}^{\pi, t, x}, s\right) \mid \mathcal{F}_{t}\right] \leq U(x, t), \quad \forall 0 \leq t \leq s<\infty, x \in \mathbb{R},
$$


and there exists an optimal $\pi^{*} \in \mathcal{A}$ such that

$$
\mathbb{E}\left[U\left(X_{s}^{\pi^{*}, t, x}, s\right) \mid \mathcal{F}_{t}\right]=U(x, t), \quad \forall 0 \leq t \leq s<\infty, x \in \mathbb{R},
$$

Moreover, a forward investment performance process $\{U(\omega, x, t)\}_{\omega \in \Omega, x \in \mathbb{R}, t \geq 0}$ is called an exponential forward investment performance process, if there exist a constant $\gamma>0$ and an $\mathbb{F}$-progressively measurable process $K=\left\{K_{t}\right\}_{t \geq 0}$ such that

$$
U(x, t)=-e^{-\gamma x+K_{t}}, \quad \forall x \in \mathbb{R}, t \geq 0 .
$$

Without loss of generality, we assume that the generic risk positions are introduced at the initial time $t=0$ to simplify the representation in the definition of the forward entropic risk measures defined by Zariphopoulou and Žitković (2010):

Definition 3.2.2. Let $\{U(\omega, x, t)\}_{\omega \in \Omega, x \in \mathbb{R}, t \geq 0}$ be an exponential forward investment performance process. Let $T \geq 0$ and $\xi_{T} \in \mathcal{L}^{\infty}\left(\mathcal{F}_{T}\right)$. The $T$-normalized forward entropic risk measure of $\xi_{T}$ is a family of risk measures $\left\{\rho_{t}\left(\xi_{T} ; T\right)\right\}_{t \in[0, T]}$, where, for each $t \in[0, T]$, the risk measure $\rho_{t}\left(\xi_{T} ; T\right)$ is defined as the $\mathcal{F}_{t}$-measurable random variable which satisfies the indifference condition:

$$
\underset{\pi \in \mathcal{A}}{\operatorname{ess} \sup } \mathbb{E}\left[U\left(\rho_{t}\left(\xi_{T} ; T\right)+X_{T}^{\pi, t, x}+\xi_{T}, T\right) \mid \mathcal{F}_{t}\right]=\underset{\pi \in \mathcal{A}}{\operatorname{ess} \sup } \mathbb{E}\left[U\left(X_{T}^{\pi, t, x}, T\right) \mid \mathcal{F}_{t}\right], \quad \forall x \in \mathbb{R} .
$$

Definition 3.2.3. Let $\xi \in \mathcal{L}$. Define

$$
T_{\xi} \triangleq \inf \left\{T \geq 0: \xi \in \mathcal{F}_{T}\right\}
$$

The forward entropic risk measure of $\xi$ is a family of risk measures $\left\{\rho_{t}(\xi)\right\}_{t \in\left[0, T_{\xi}\right]}$, where, for each $t \in\left[0, T_{\xi}\right]$, the risk measure $\rho_{t}(\xi)$ is defined by

$$
\rho_{t}(\xi) \triangleq \rho_{t}\left(\xi ; T_{\xi}\right)
$$

One should realize that the definition of $T$-normalized forward entropic risk measures is the same as that of the classical entropic risk measures by replacing the 
exponential utility function by the exponential forward investment performance process. However, we emphasize that since the performance process is defined for all times $t \geq 0$, the forward entropic risk measure can evaluate a risk position with arbitrage maturity. On the other hand, since the classical exponential value function is defined only on a finite horizon, the entropic risk measure in the classical framework cannot evaluate risk positions with arbitrary maturities.

Finally, one should also realize that both the forward investment performance processes and hence the forward entropic risk measures depend on the financial market environment. Therefore, we shall properly define the market model that shall be adapted, namely, the stochastic factor market model, in the next section.

\subsection{Stochastic Factor Market Model}

We consider a financial market model of a risk-free asset $S^{0}=\left\{S_{t}^{0}\right\}_{t \geq 0}$ with zero interest rate and $n$ risky stocks $S=\left\{\left(S_{t}^{1}, S_{t}^{2}, \ldots, S_{t}^{n}\right)^{t r}\right\}_{t \geq 0}$, with $n \leq d$, where $d$ is the dimension of the Brownian motion. The stock price processes solve, for $i=1,2, \ldots, n$,

$$
\frac{d S_{t}^{i}}{S_{t}^{i}}=b^{i}\left(V_{t}\right) d t+\sigma^{i}\left(V_{t}\right) d W_{t}, \quad \forall t \geq 0,
$$

while the $d$-dimensional stochastic factors $V=\left\{\left(V_{t}^{1}, V_{t}^{2}, \ldots, V_{t}^{d}\right)^{t r}\right\}_{t \geq 0}$ solves

$$
d V_{t}=\eta\left(V_{t}\right) d t+\kappa d W_{t}, \quad \forall t \geq 0 .
$$

We shall use the superscript $t, v$, whenever appropriate, to indicate the dependence on the initial condition $v \in \mathbb{R}^{d}$ of the stochastic factors at the initial time $t \geq 0$.

We assume the following hold in the remaining of this chapter.

\section{Assumption 3.3.1.}

(i) The drift and volatility coefficients $\left\{b^{i}(v)\right\}_{v \in \mathbb{R}^{d}} \in \mathbb{R}$ and $\left\{\sigma^{i}(v)\right\}_{v \in \mathbb{R}^{d}} \in \mathbb{R}^{1 \times d}$ are uniformly bounded in $v \in \mathbb{R}^{d}$; 
(ii) The volatility matrix

$$
\{\sigma(v)\}_{v \in \mathbb{R}^{d}}=\left\{\left(\sigma^{1}(v), \sigma^{2}(v), \ldots, \sigma^{n}(v)\right)^{t r}\right\}_{v \in \mathbb{R}^{d}}
$$

has full row rank $n$;

(iii) The market price of risk

$$
\{\theta(v)\}_{v \in \mathbb{R}^{d}}=\left\{\sigma(v)^{t r}\left(\sigma(v) \sigma(v)^{t r}\right)^{-1} b(v)\right\}_{v \in \mathbb{R}^{d}}
$$

which exist by (ii), is uniformly bounded and Lipschitz continuous in $v \in \mathbb{R}^{d}$.

\section{Assumption 3.3.2.}

(i) The drift coefficient $\{\eta(v)\}_{v \in \mathbb{R}^{d}} \in \mathbb{R}^{d}$ satisfies a dissipative condition, namely, there exists a constant $C_{\eta}>0$ such that

$$
\left(\eta\left(v_{1}\right)-\eta\left(v_{2}\right)\right)^{t r}\left(v_{1}-v_{2}\right) \leq-C_{\eta}\left|v_{1}-v_{2}\right|^{2}, \quad \forall v_{1}, v_{2} \in \mathbb{R}^{d}
$$

and

$$
C_{\eta}>C_{v}>0
$$

where the positive constant $C_{v}$ is a locally Lipschitz constant for the driver in the upcoming ergodic BSDE (3.4.8).

(ii) The constant volatility matrix $\kappa \in \mathbb{R}^{d \times d}$ is positive definite and normalized such that $|\kappa|=1$.

Denote $\tilde{\pi}=\left\{\left(\tilde{\pi}_{t}^{1}, \tilde{\pi}_{t}^{2}, \ldots, \tilde{\pi}_{t}^{n}\right)^{t r}\right\}_{t \geq 0}$ as the (discounted) amount investing into the $n$ risky assets. Then, in this stochastic factor market environment, the (discounted) wealth process $X^{\tilde{\pi}}=\left\{X_{t}^{\tilde{\pi}}\right\}_{t \geq 0}$ satisfies

$$
d X_{t}^{\tilde{\pi}}=\sum_{i=1}^{n} \tilde{\pi}_{t}^{i} \frac{d S_{t}^{i}}{S_{t}^{i}}=\tilde{\pi}^{t r} \sigma\left(V_{t}\right)\left(\theta\left(V_{t}\right) d t+d W_{t}\right), \quad \forall t \geq 0
$$

Denote $\pi=\left\{\left(\pi_{t}^{1}, \pi_{t}^{2}, \ldots, \pi_{t}^{n}\right)^{t r}\right\}_{t \geq 0}$ as the investment strategies rescaled by the volatility:

$$
\pi_{t}^{t r}=\tilde{\pi}_{t}^{t r} \sigma\left(V_{t}\right), \quad \forall t \geq 0
$$


Therefore, the wealth process $X^{\pi}=\left\{X_{t}^{\pi}\right\}_{t \geq 0}$ satisfies

$$
d X_{t}^{\pi}=\pi_{t}^{t r}\left(\theta\left(V_{t}\right) d t+d W_{t}\right), \quad \forall t \geq 0
$$

Denote $\mathcal{A}[0, t]$ as the set of admissible investment strategies defined for times $s \in[0, t]:$

$$
\mathcal{A}[0, t]=\left\{\pi=\left\{\pi_{s}\right\}_{s \in[0, t]}: \pi \in \mathcal{L}_{B M O}^{2}[0, t] \text { and } \pi_{s} \in \Pi, \forall s \in[0, t]\right\}
$$

where

$$
\begin{aligned}
& \mathcal{L}_{B M O}^{2}[0, t]=\left\{\pi=\left\{\pi_{s}\right\}_{s \in[0, t]}: \pi \text { is } \mathbb{F}\right. \text {-progessively measureable and } \\
& \left.\operatorname{ess~sup}_{\tau \in \mathcal{T}[0, t]} \mathbb{E}\left[\int_{\tau}^{t}\left|\pi_{s}\right|^{2} d s \mid \mathcal{F}_{\tau}\right]<\infty\right\}
\end{aligned}
$$

$\mathcal{T}[0, t]$ is the set of $\mathbb{F}$-stopping time $\tau \in[0, t]$, and $\Pi$ is a closed and convex subset of $\mathbb{R}^{d}$. Then, the set $\mathcal{A}$ of admissible investment strategies defined for times $t \geq 0$ is defined by

$$
\mathcal{A}=\cup_{t \geq 0} \mathcal{A}[0, t]
$$

\subsection{Ergodic BSDE Representation for Exponen- tial Forward Investment Performance Pro- cesses}

In this section, we recall recent results on the ergodic BSDE representation for the exponential forward investment performance processes by Liang and Zariphopoulou (2017).

Define a function $F: \mathbb{R}^{d} \times \mathbb{R}^{d} \rightarrow \mathbb{R}$ by

$$
F(v, z) \triangleq \frac{\gamma^{2}}{2} \operatorname{dist}^{2}\left\{\Pi, \frac{z+\theta(v)}{\gamma}\right\}-\frac{1}{2}|z+\theta(v)|^{2}+\frac{1}{2}|z|^{2}, \quad \forall v \in \mathbb{R}^{d}, z \in \mathbb{R}^{d}
$$


By Assumption 3.3.1 and the Lipschitz continuous property of the distance function, there exist constants $C_{v}>0$ and $C_{z}>0$ such that, for any $v_{1}, v_{2}, z_{1}, z_{2} \in \mathbb{R}^{d}$,

$$
\left|F\left(v_{1}, z_{1}\right)-F\left(v_{2}, z_{1}\right)\right| \leq C_{v}\left(1+\left|z_{1}\right|\right)\left|v_{1}-v_{2}\right|,
$$

and

$$
\left|F\left(v_{1}, z_{1}\right)-F\left(v_{1}, z_{2}\right)\right| \leq C_{z}\left(1+\left|z_{1}\right|+\left|z_{2}\right|\right)\left|z_{1}-z_{2}\right|
$$

Proposition 3.4.1. Suppose that Assumptions 3.3.1 and 3.3.2 hold. Then the ergodic $B S D E$

$$
d Y_{t}=\left(-F\left(V_{t}, Z_{t}\right)+\lambda\right) d t+Z_{t}^{t r} d W_{t}, \quad \forall t \geq 0
$$

admits a unique Markovian solution $\left(Y=\left\{Y_{t}\right\}_{t \geq 0}, Z=\left\{Z_{t}\right\}_{t \geq 0}, \lambda\right)$. Specifically, there exist a unique $\lambda \in \mathbb{R}$, a function $y: \mathbb{R}^{d} \rightarrow \mathbb{R}$ such that

$$
Y_{t}=y\left(V_{t}\right), \quad \forall t \geq 0
$$

and a function $z: \mathbb{R}^{d} \rightarrow \mathbb{R}^{d}$ such that

$$
Z_{t}=z\left(V_{t}\right), \quad \forall t \geq 0
$$

The function y is unique up to an additive constant, and has at most linear growth, while the function $z$ is uniformly bounded:

$$
|z(v)| \leq \frac{C_{v}}{C_{\eta}-C_{v}}, \quad \forall v \in \mathbb{R}^{d}
$$

Moreover,

$$
|\nabla y(v)| \leq \frac{C_{v}}{C_{\eta}-C_{v}}, \quad \forall v \in \mathbb{R}^{d} .
$$

Proof. For the proof, see Proposition 10 in Liang and Zariphopoulou (2017).

While there exists the unique solution $(Y, Z, \lambda)$ in the stochastic factor form $(y, z, \lambda)$, the ergodic BSDE (3.4.8) admits multiple non-Markovian solutions. In particular, we may choose

$$
Z_{t}=z^{0}\left(V_{t}\right), \quad \forall t \geq 0
$$


for any differentiable and bounded function $z^{0}: \mathbb{R}^{d} \rightarrow \mathbb{R}^{d}$ with bounded first order derivative, and any pair $(Y, \lambda)$ such that the ergodic BSDE 3.4.8 holds. Then, $\left(Y, z^{0}, \lambda\right)$ is a non-Markovian solution to the ergodic BSDE (3.4.8).

Theorem 3.4.2. Suppose that Assumptions 3.3.1 and 3.3.2 hold. Let $(Y, Z, \lambda)$ be a solution to Equation (3.4.8) such that $Z$ is uniformly bounded. Then the process $\{U(\omega, x, t)\}_{\omega \in \Omega, x \in \mathbb{R}, t \geq 0}$ defined by

$$
U(x, t)=-e^{-\gamma x+Y_{t}-\lambda t}, \quad \forall x \in \mathbb{R}, t \geq 0,
$$

where $\gamma>0$, is an exponential forward investment performance process, with the associated optimal investment strategy

$$
\pi_{t}^{*}=\mathcal{P}_{\Pi}\left(\frac{Z_{t}+\theta\left(V_{t}\right)}{\gamma}\right), \quad \forall t \geq 0 .
$$

Proof. For the proof, see Theorem 11 in Liang and Zariphopoulou (2017).

By Proposition 3.4.1 and Theorem 3.4.2, we deduce that, while general exponential forward investment performance processes (3.4.10) are not unique, there exists a unique exponential forward investment performance process in the stochastic factor form:

$$
U(x, t)=-e^{-\gamma x+y\left(V_{t}\right)-\lambda t}, \quad \forall x \in \mathbb{R}, t \geq 0,
$$

with the pair $(y, \lambda)$ given in Proposition 3.4.1.

In the remaining of this chapter, we consider the exponential forward investment performance processes with the form of (3.4.10).

\subsection{BSDE Representation of Forward Entropic Risk Measures}

This section provides one of the main results of this chapter, which is the representation of the forward entropic risk measures using the solutions of associated 
BSDE and ergodic BSDE. The main idea is to express the forward entropic risk measures as the solutions of traditional BSDEs whose drivers, however, depend on the solution of the ergodic BSDE (3.4.8). This dependence follows from the fact that the ergodic BSDE (3.4.8) was used to construct the exponential forward investment performance process 3.4 .10 that enters into the indifference condition 3.2 .2 in Definition 3.2 .2 .

Define a function $G: \mathbb{R}^{d} \times \mathbb{R}^{d} \times \mathbb{R}^{d} \rightarrow \mathbb{R}$ by

$$
G(v, z, \hat{z}) \triangleq \frac{1}{\gamma}(F(v, z+\gamma \hat{z})-F(v, z)), \quad \forall v \in \mathbb{R}^{d}, z \in \mathbb{R}^{d}, \hat{z} \in \mathbb{R}^{d}
$$

By locally Lipschitz continuous property of $F$ in (3.4.7), $G$ satisfies, for any $v, z, \hat{z}_{1}, \hat{z}_{2} \in \mathbb{R}^{d}$

$$
\left|G\left(v, z, \hat{z}_{1}\right)-G\left(v, z, \hat{z}_{2}\right)\right| \leq C_{z}\left(1+2|z|+\gamma\left|\hat{z}_{1}\right|+\gamma\left|\hat{z}_{2}\right|\right)\left|\hat{z}_{1}-\hat{z}_{2}\right| \cdot
$$

Theorem 3.5.1. Suppose that Assumptions 3.3.1 and 3.3.2 hold, and that the solution $Z$ in the ergodic BSDE (3.4.8) is uniformly bounded. Let $T \geq 0$ and $\xi_{T} \in \mathcal{L}^{\infty}\left(\mathcal{F}_{T}\right)$. Then,

(i) the finite horizon BSDE

$$
Y_{t}^{T, \xi}=-\xi_{T}+\int_{t}^{T} G\left(V_{u}, Z_{u}, Z_{u}^{T, \xi}\right) d u-\int_{t}^{T}\left(Z_{u}^{T, \xi}\right)^{t r} d W_{u}, \quad \forall t \in[0, T],
$$

admits a unique solution $\left(Y^{T, \xi}=\left\{Y_{t}^{T, \xi}\right\}_{t \in[0, T]}, Z^{T, \xi}=\left\{Z_{t}^{T, \xi}\right\}_{t \in[0, T]}\right)$ such that $Y^{T, \xi}$ is uniformly bounded and $Z^{T, \xi} \in \mathcal{L}_{B M O}^{2}[0, T]$;

(ii) the forward entropic risk measure of $\xi_{T}$ can be represented by the solution $Y^{T, \xi}$ in the BSDE (3.5.14), i.e.,

$$
\rho_{t}\left(\xi_{T}\right)=Y_{t}^{T, \xi}, \quad \forall t \in[0, T]
$$

Proof. 
(i) Since the solution $Z$ in the ergodic BSDE 3.4.8 is uniformly bounded, the function $G$ is locally Lipschitz continuous in $\hat{z}$ by (3.5.13). By Theorem 2.3 and 2.6 in Kobylanski (2000) and Theorem 7 in Hu et al. (2005), together with $\xi_{T} \in \mathcal{L}^{\infty}\left(\mathcal{F}_{T}\right)$, the first assertion follows.

(ii) Let $t \in[0, T]$ and $x \in \mathbb{R}$.

By the condition $(3.2 .1)$ of the forward investment performance process and the form of the exponential forward investment performance process 3.4.10,

$$
\begin{aligned}
\underset{\pi \in \mathcal{A}}{\operatorname{ess} \sup } \mathbb{E}\left[U\left(X_{T}^{\pi, t, x}, T\right) \mid \mathcal{F}_{t}\right] & =U(x, t) \\
& =-e^{-\gamma x+Y_{t}-\lambda t}
\end{aligned}
$$

which is the right hand side of the indifference condition (3.2.2).

By the form of the exponential forward investment performance process 3.4.10 and the dynamics for the wealth process 3.3.4,

$$
\begin{aligned}
& \underset{\pi \in \mathcal{A}}{\operatorname{ess} \sup _{i}} \mathbb{E}\left[U\left(\rho_{t}\left(\xi_{T}\right)+X_{T}^{\pi, t, x}+\xi_{T}, T\right) \mid \mathcal{F}_{t}\right] \\
= & e^{-\gamma \rho_{t}\left(\xi_{T}\right)} \operatorname{ess} \sup _{\pi \in \mathcal{A}} \mathbb{E}\left[-e^{-\gamma\left(x+\int_{t}^{T} \pi_{u}^{t r}\left(\theta\left(V_{u}\right) d u+d W_{u}\right)\right)+Y_{T}-\lambda T-\gamma \xi_{T}} \mid \mathcal{F}_{t}\right],
\end{aligned}
$$

which is the left hand side of the indifference condition 3.2.2. Define

$$
R_{s}^{\pi} \triangleq-e^{-\gamma\left(x+\int_{t}^{s} \pi_{u}^{t r}\left(\theta\left(V_{u}\right) d u+d W_{u}\right)\right)+Y_{s}-\lambda s+\gamma Y_{s}^{T, \xi}}, \quad \forall s \in[t, T]
$$

We shall show that $R^{\pi}=\left\{R_{s}^{\pi}\right\}_{s \in[t, T]}$ is a super-martingale for any $\pi \in \mathcal{A}$, and in particular, is a martingale for

$$
\pi_{s}^{*, \xi}=\mathcal{P}_{\Pi}\left(Z_{s}^{T, \xi}+\frac{Z_{s}+\theta\left(V_{s}\right)}{\gamma}\right), \quad \forall s \in[t, T],
$$

which clearly lies in $\mathcal{A}$, since $Z^{T, \xi} \in \mathcal{L}_{B M O}^{2}[0, T]$ and $Z$ and $\theta$ are uniformly bounded. 
If these hold, for any $\pi \in \mathcal{A}, \mathbb{E}\left[R_{T}^{\pi} \mid \mathcal{F}_{t}\right] \leq R_{t}^{\pi}$, while $\mathbb{E}\left[R_{T}^{\pi^{*, \xi}} \mid \mathcal{F}_{t}\right]=R_{t}^{\pi}$. In other words, since $Y_{T}^{T, \xi}=-\xi_{T}$, for any $\pi \in \mathcal{A}$,

$$
\mathbb{E}\left[-e^{-\gamma\left(x+\int_{t}^{T} \pi_{u}^{t r}\left(\theta\left(V_{u}\right) d u+d W_{u}\right)\right)+Y_{T}-\lambda T-\gamma \xi_{T}} \mid \mathcal{F}_{t}\right] \leq-e^{-\gamma x+Y_{t}-\lambda t+\gamma Y_{t}^{T, \xi}}
$$

while

$$
\mathbb{E}\left[-e^{-\gamma\left(x+\int_{t}^{T}\left(\pi_{u}^{*, \xi}\right)^{\operatorname{tr}}\left(\theta\left(V_{u}\right) d u+d W_{u}\right)\right)+Y_{T}-\lambda T-\gamma \xi_{T}} \mid \mathcal{F}_{t}\right]=-e^{-\gamma x+Y_{t}-\lambda t+\gamma Y_{t}^{T, \xi}}
$$

Therefore, the left hand side of the indifference condition $(3.2 .2)$ becomes

$$
-e^{-\gamma \rho_{t}\left(\xi_{T}\right)-\gamma x+Y_{t}-\lambda t+\gamma Y_{t}^{T, \xi}}
$$

Finally, the indifference condition $(3.2 .2)$ becomes

$$
-e^{-\gamma \rho_{t}\left(\xi_{T}\right)-\gamma x+Y_{t}-\lambda t+\gamma Y_{t}^{T, \xi}}=-e^{-\gamma x+Y_{t}-\lambda t},
$$

and hence the second assertion follows.

Let $\pi \in \mathcal{A}$. Let $s \in[t, T]$ and $r \in[t, s]$. Note that

$$
\begin{aligned}
& -\gamma\left(x+\int_{t}^{s} \pi_{u}^{t r}\left(\theta\left(V_{u}\right) d u+d W_{u}\right)\right)+Y_{s}-\lambda s+\gamma Y_{s}^{T, \xi} \\
= & -\gamma\left(x+\int_{t}^{r} \pi_{u}^{t r}\left(\theta\left(V_{u}\right) d u+d W_{u}\right)\right)+Y_{r}-\lambda r+\gamma Y_{r}^{T, \xi} \\
& -\gamma \int_{r}^{s} \pi_{u}^{t r}\left(\theta\left(V_{u}\right) d u+d W_{u}\right)+\left(Y_{s}-Y_{r}\right)-\lambda(s-r)+\gamma\left(Y_{s}^{T, \xi}-Y_{r}^{T, \xi}\right) .
\end{aligned}
$$

By the ergodic BSDE 3.4.8,

$$
\left(Y_{s}-Y_{r}\right)-\lambda(s-r)=-\int_{r}^{s} F\left(V_{u}, Z_{u}\right) d u+\int_{r}^{s} Z_{u}^{t r} d W_{u}
$$

By the finite horizon BSDE (3.5.14),

$$
Y_{s}^{T, \xi}-Y_{r}^{T, \xi}=-\frac{1}{\gamma} \int_{r}^{s}\left(F\left(V_{u}, Z_{u}+\gamma Z_{u}^{T, \xi}\right)-F\left(V_{u}, Z_{u}\right)\right) d u+\int_{r}^{s}\left(Z_{u}^{T, \xi}\right)^{t r} d W_{u}
$$


Therefore,

$$
\begin{aligned}
& \mathbb{E}\left[R_{s}^{\pi} \mid \mathcal{F}_{r}\right] \\
= & \mathbb{E}\left[-e^{-\gamma\left(x+\int_{t}^{s} \pi_{u}^{t r}\left(\theta\left(V_{u}\right) d u+d W_{u}\right)\right)+Y_{s}-\lambda s+\gamma Y_{s}^{T, \xi}} \mid \mathcal{F}_{r}\right] \\
= & -e^{-\gamma\left(x+\int_{t}^{r} \pi_{u}^{t r}\left(\theta\left(V_{u}\right) d u+d W_{u}\right)\right)+Y_{r}-\lambda r+\gamma Y_{r}^{T, \xi}} \\
& \times \mathbb{E}\left[e^{\int_{r}^{s}\left(-\gamma \pi_{u}^{t r} \theta\left(V_{u}\right)-F\left(V_{u}, Z_{u}+\gamma Z_{u}^{T, \xi}\right)\right) d u+\int_{r}^{s}\left(-\gamma \pi_{u}+Z_{u}+\gamma Z_{u}^{T, \xi}\right)^{t r} d W_{u}} \mid \mathcal{F}_{r}\right] \\
= & R_{r}^{\pi} \times \mathbb{E}\left[e^{\int_{r}^{s}\left(-\gamma \pi_{u}^{t r} \theta\left(V_{u}\right)-F\left(V_{u}, Z_{u}+\gamma Z_{u}^{T, \xi}\right)\right) d u+\int_{r}^{s}\left(-\gamma \pi_{u}+Z_{u}+\gamma Z_{u}^{T, \xi}\right)^{t r} d W_{u}} \mid \mathcal{F}_{r}\right] .
\end{aligned}
$$

Define

$$
N_{s} \triangleq \int_{0}^{s}\left(-\gamma \pi_{u}+Z_{u}+\gamma Z_{u}^{T, \xi}\right)^{t r} d W_{u}, \quad \forall s \in[0, T]
$$

Since $\pi, Z^{T, \xi} \in \mathcal{L}_{B M O}^{2}[0, T]$ and $Z$ is uniformly bounded, the stochastic process $N=\left\{N_{s}\right\}_{s \in[0, T]}$ is a BMO-martingale. Therefore, the Dolean exponential $\mathcal{E}(N)=\left\{\mathcal{E}(N)_{s}\right\}_{s \in[0, T]}$ of $N$, where

$$
\mathcal{E}(N)_{s}=e^{N_{s}-\frac{1}{2}\langle N .\rangle_{s}}, \quad \forall s \in[0, T]
$$

is a uniformly integrable martingale. Hence, we can define a $\mathbb{P}$-equivalent probability measure $\mathbb{Q}^{\pi}$ on $\mathcal{F}_{T}$ by the Radon-Nikodym derivative:

$$
\left.\frac{d \mathbb{Q}^{\pi}}{d \mathbb{P}}\right|_{\mathcal{F}_{T}}=\mathcal{E}(N)_{T},
$$

and thus

$$
\left.\frac{d \mathbb{Q}^{\pi}}{d \mathbb{P}}\right|_{\mathcal{F}_{s}}=\mathcal{E}(N)_{s}, \quad \forall s \in[0, T] .
$$

By the definition of $N$ and Dolean exponential $\mathcal{E}(N)$,

$$
e^{\int_{r}^{s}\left(-\gamma \pi_{u}+Z_{u}+\gamma Z_{u}^{T, \xi}\right)^{t r} d W_{u}}=e^{\frac{1}{2} \int_{r}^{s}\left|-\gamma \pi_{u}+Z_{u}+\gamma Z_{u}^{T, \xi}\right|^{2} d u} \cdot \frac{\mathcal{E}(N)_{s}}{\mathcal{E}(N)_{r}} .
$$


Therefore,

$$
\begin{aligned}
& \mathbb{E}\left[e^{\int_{r}^{s}\left(-\gamma \pi_{u}^{t r} \theta\left(V_{u}\right)-F\left(V_{u}, Z_{u}+\gamma Z_{u}^{T, \xi}\right)\right) d u+\int_{r}^{s}\left(-\gamma \pi_{u}+Z_{u}+\gamma Z_{u}^{T, \xi}\right)^{t r} d W_{u}} \mid \mathcal{F}_{r}\right] \\
= & \mathbb{E}\left[e^{\int_{r}^{s}\left(-\gamma \pi_{u}^{t r} \theta\left(V_{u}\right)-F\left(V_{u}, Z_{u}+\gamma Z_{u}^{T, \xi}\right)\right) d u+\frac{1}{2} \int_{r}^{s}\left|-\gamma \pi_{u}+Z_{u}+\gamma Z_{u}^{T, \xi}\right|^{2} d u} \cdot \frac{\mathcal{E}(N)_{s}}{\mathcal{E}(N)_{r}} \mid \mathcal{F}_{r}\right] \\
= & \mathbb{E}^{\mathbb{Q}^{\pi}}\left[e^{\int_{r}^{s}\left(\left(-\gamma \pi_{u}^{t r} \theta\left(V_{u}\right)+\frac{1}{2}\left|-\gamma \pi_{u}+Z_{u}+\gamma Z_{u}^{T, \xi}\right|^{2}\right)-F\left(V_{u}, Z_{u}+\gamma Z_{u}^{T, \xi}\right)\right) d u} \mid \mathcal{F}_{r}\right] .
\end{aligned}
$$

Simple calculations yield that, for any $u \in[r, s]$,

$$
\begin{aligned}
& -\gamma \pi_{u}^{t r} \theta\left(V_{u}\right)+\frac{1}{2}\left|-\gamma \pi_{u}+Z_{u}+\gamma Z_{u}^{T, \xi}\right|^{2} \\
= & \frac{\gamma^{2}}{2}\left|\pi_{u}-\left(Z_{u}^{T, \xi}+\frac{Z_{u}+\theta\left(V_{u}\right)}{\gamma}\right)\right|^{2}-\frac{1}{2}\left|Z_{u}+\gamma Z_{u}^{T, \xi}+\theta\left(V_{u}\right)\right|^{2}+\frac{1}{2}\left|Z_{u}+\gamma Z_{u}^{T, \xi}\right|^{2} \\
\geq & \frac{\gamma^{2}}{2} \operatorname{dist}^{2}\left\{\Pi, Z_{u}^{T, \xi}+\frac{Z_{u}+\theta\left(V_{u}\right)}{\gamma}\right\}-\frac{1}{2}\left|Z_{u}+\gamma Z_{u}^{T, \xi}+\theta\left(V_{u}\right)\right|^{2}+\frac{1}{2}\left|Z_{u}+\gamma Z_{u}^{T, \xi}\right|^{2} \\
= & F\left(V_{u}, Z_{u}+\gamma Z_{u}^{T, \xi}\right) .
\end{aligned}
$$

Hence,

$$
\begin{aligned}
& \mathbb{E}\left[e^{\int_{r}^{s}\left(-\gamma \pi_{u}^{t r} \theta\left(V_{u}\right)-F\left(V_{u}, Z_{u}+\gamma Z_{u}^{T, \xi}\right)\right) d u+\int_{r}^{s}\left(-\gamma \pi_{u}+Z_{u}+\gamma Z_{u}^{T, \xi}\right)^{t r} d W_{u}} \mid \mathcal{F}_{r}\right] \\
= & \mathbb{E}^{\mathbb{Q}^{\pi}}\left[e^{\int_{r}^{s}\left(\left(-\gamma \pi_{u}^{t r} \theta\left(V_{u}\right)+\frac{1}{2}\left|-\gamma \pi_{u}+Z_{u}+\gamma Z_{u}^{T, \xi}\right|^{2}\right)-F\left(V_{u}, Z_{u}+\gamma Z_{u}^{T, \xi}\right)\right) d u} \mid \mathcal{F}_{r}\right] \\
\geq & 1 .
\end{aligned}
$$

Therefore, since $R^{\pi}$ is negative, by 3.5 .15 ,

$$
\mathbb{E}\left[R_{s}^{\pi} \mid \mathcal{F}_{r}\right] \leq R_{r}^{\pi}
$$

Moreover, if

$$
\pi_{s}=\mathcal{P}_{\Pi}\left(Z_{s}^{T, \xi}+\frac{Z_{s}+\theta\left(V_{s}\right)}{\gamma}\right), \quad \forall s \in[t, T]
$$

then, for any $u \in[r, s]$,

$$
-\gamma \pi_{u}^{t r} \theta\left(V_{u}\right)+\frac{1}{2}\left|-\gamma \pi_{u}+Z_{u}+\gamma Z_{u}^{T, \xi}\right|^{2}=F\left(V_{u}, Z_{u}+\gamma Z_{u}^{T, \xi}\right),
$$


and thus

$$
\mathbb{E}\left[e^{\int_{r}^{s}\left(-\gamma \pi_{u}^{t r} \theta\left(V_{u}\right)-F\left(V_{u}, Z_{u}+\gamma Z_{u}^{T, \xi}\right)\right) d u+\int_{r}^{s}\left(-\gamma \pi_{u}+Z_{u}+\gamma Z_{u}^{T, \xi}\right)^{t r} d W_{u}} \mid \mathcal{F}_{r}\right]=1,
$$

and

$$
\mathbb{E}\left[R_{s}^{\pi} \mid \mathcal{F}_{r}\right]=R_{r}^{\pi}
$$

Therefore, we showed the claim that $R^{\pi}=\left\{R_{s}^{\pi}\right\}_{s \in[t, T]}$ is a super-martingale for any $\pi \in \mathcal{A}$, and in particular, is a martingale for

$$
\pi_{s}^{*, \xi}=\mathcal{P}_{\Pi}\left(Z_{s}^{T, \xi}+\frac{Z_{s}+\theta\left(V_{s}\right)}{\gamma}\right), \quad \forall s \in[t, T],
$$

Before closing this section, we make several important remarks.

Firstly, by Proposition 3.4.1, there exists at least one solution $Z$ of the ergodic BSDE (3.4.8) which is uniformly bounded, which justifies the validity of the condition that the solution $Z$ is uniformly bounded in Theorem 3.5.1.

Secondly, it is natural to expect that the forward entropic risk measures could be represented by the solution of BSDEs, since the indifference condition 3.2 .2 is, by nature, set backwards in time. However, the finite horizon BSDEs (3.5.14) of the forward entropic risk measures are different from those in the classical exponential utility framework, see, for instance, $\mathrm{Hu}$ et al. (2005). In particular, the drivers of finite horizon BSDEs (3.5.14 depend on the solutions $Z$ from the ergodic BSDEs 3.4 .8 for the exponential forward investment performance processes 3.4 .10 .

Thirdly, using the BSDE representation of the forward entropic risk measures, we can show that they are maturity independent convex risk measures, as promised in Section 3.1. (i) By the definition of forward entropic risk measure in (3.2.2), $\rho_{t}(0)=0$. If $\xi_{T} \geq 0$, by the comparison principle of the finite horizon BSDE on 
the terminal condition,

$$
\rho_{t}\left(\xi_{T}\right)=Y_{t}^{T, \xi} \leq Y_{t}^{T, 0}=\rho_{t}(0)=0
$$

which shows the property of anti-positivity. (ii) Let $m \in \mathcal{L}^{\infty}\left(\mathcal{F}_{t}\right)$. Then, $\rho_{t}\left(\xi_{T}-\right.$ $m)=Y_{t}^{T, \xi-m}$, where $Y^{T, \xi-m}$ is the solution of the BSDE:

$$
Y_{t}^{T, \xi-m}=-\left(\xi_{T}-m\right)+\int_{t}^{T} G\left(V_{u}, Z_{u}, Z_{u}^{T, \xi-m}\right) d u-\int_{t}^{T}\left(Z_{u}^{T, \xi-m}\right)^{t r} d W_{u}
$$

which is equivalent to that

$$
Y_{t}^{T, \xi-m}-m=-\xi_{T}+\int_{t}^{T} G\left(V_{u}, Z_{u}, Z_{u}^{T, \xi-m}\right) d u-\int_{t}^{T}\left(Z_{u}^{T, \xi-m}\right)^{t r} d W_{u} .
$$

Therefore, $\left(Y^{T, \xi-m}-m, Z^{T, \xi-m}\right)$ solves the BSDE:

$$
Y_{t}^{T, \xi}=-\xi_{T}+\int_{t}^{T} G\left(V_{u}, Z_{u}, Z_{u}^{T, \xi}\right) d u-\int_{t}^{T}\left(Z_{u}^{T, \xi}\right)^{t r} d W_{u}
$$

By the uniqueness of solution, $Y^{T, \xi-m}-m=Y^{T, \xi}$ and $Z^{T, \xi-m}=Z^{T, \xi}$. Since $\rho_{t}\left(\xi_{T}\right)=Y_{t}^{T, \xi}$,

$$
\rho_{t}\left(\xi_{T}-m\right)=Y_{t}^{T, \xi-m}=Y_{t}^{T, \xi}+m=\rho_{t}\left(\xi_{T}\right)+m
$$

which shows the property of translation invariance. (iii) Since $\Pi$ is a convex subset in $\mathbb{R}^{d}$, the distance function to the convex set $\Pi$ in 3.5 .12 is also convex. Therefore, for any $(v, z) \in \mathbb{R}^{d} \times \mathbb{R}^{d}$, the function $G$ in 3.5 .12 is convex in $\hat{z}$. Let $T \geq 0, \xi_{T}, \xi_{T}^{\prime} \in \mathcal{L}^{\infty}\left(\mathcal{F}_{T}\right), t \in[0, T]$, and $\alpha \in[0,1]$. Then, $\rho_{t}\left(\alpha \xi_{T}+(1-\alpha) \xi_{T}^{\prime}\right)=$ $Y_{t}^{T, \alpha \xi+(1-\alpha) \xi^{\prime}}$, where $Y^{T, \alpha \xi+(1-\alpha) \xi^{\prime}}$ is the solution of the BSDE:

$$
\begin{aligned}
Y_{t}^{T, \alpha \xi+(1-\alpha) \xi^{\prime}}= & -\left(\alpha \xi_{T}+(1-\alpha) \xi_{T}^{\prime}\right) \\
& +\int_{t}^{T} G\left(V_{u}, Z_{u}, Z_{u}^{T, \alpha \xi+(1-\alpha) \xi^{\prime}}\right) d u-\int_{t}^{T}\left(Z_{u}^{T, \alpha \xi+(1-\alpha) \xi^{\prime}}\right)^{t r} d W_{u}
\end{aligned}
$$

Also, $\rho_{t}\left(\xi_{T}\right)=Y_{t}^{T, \xi}$, where $Y^{T, \xi}$ solves

$$
Y_{t}^{T, \xi}=-\xi_{T}+\int_{t}^{T} G\left(V_{u}, Z_{u}, Z_{u}^{T, \xi}\right) d u-\int_{t}^{T}\left(Z_{u}^{T, \xi}\right)^{t r} d W_{u}
$$


while $\rho_{t}\left(\xi_{T}^{\prime}\right)=Y_{t}^{T, \xi^{\prime}}$, where $Y^{T, \xi^{\prime}}$ solves

$$
Y_{t}^{T, \xi^{\prime}}=-\xi_{T}^{\prime}+\int_{t}^{T} G\left(V_{u}, Z_{u}, Z_{u}^{T, \xi^{\prime}}\right) d u-\int_{t}^{T}\left(Z_{u}^{T, \xi^{\prime}}\right)^{t r} d W_{u} .
$$

Therefore,

$$
\begin{aligned}
\alpha Y_{t}^{T, \xi}+(1-\alpha) Y_{t}^{T, \xi^{\prime}}= & -\left(\alpha \xi_{T}+(1-\alpha) \xi_{T}^{\prime}\right) \\
& +\int_{t}^{T}\left(\alpha G\left(V_{u}, Z_{u}, Z_{u}^{T, \xi}\right)+(1-\alpha) G\left(V_{u}, Z_{u}, Z_{u}^{T, \xi^{\prime}}\right)\right) d u \\
& -\int_{t}^{T}\left(\alpha Z_{u}^{T, \xi}+(1-\alpha) Z_{u}^{T, \xi^{\prime}}\right)^{t r} d W_{u}
\end{aligned}
$$

By the convexity of the function $G$ in $\hat{z}$, for any $u \in[t, T]$,

$$
G\left(V_{u}, Z_{u}, \alpha Z_{u}^{T, \xi}+(1-\alpha) Z_{u}^{T, \xi^{\prime}}\right) \leq \alpha G\left(V_{u}, Z_{u}, Z_{u}^{T, \xi}\right)+(1-\alpha) G\left(V_{u}, Z_{u}, Z_{u}^{T, \xi^{\prime}}\right)
$$

Therefore, by the comparison principle of the finite horizon BSDE on the driver,

$$
\begin{aligned}
\rho_{t}\left(\alpha \xi_{T}+(1-\alpha) \xi_{T}^{\prime}\right) & =Y_{t}^{T, \alpha \xi+(1-\alpha) \xi^{\prime}} \\
& \leq \alpha Y_{t}^{T, \xi}+(1-\alpha) Y_{t}^{T, \xi^{\prime}} \\
& =\alpha \rho_{t}\left(\xi_{T}\right)+(1-\alpha) \rho_{t}\left(\xi_{T}^{\prime}\right)
\end{aligned}
$$

which shows the property of convexity. (iv) For any $T \geq 0, t \in[0, T]$, and $s \in[t, T]$,

$$
\rho_{t}\left(\xi_{T}\right)=Y_{t}^{T, \xi}=Y_{t}^{s,-Y_{s}^{T, \xi}}=Y_{t}^{s,-\rho_{s}\left(\xi_{T}\right)}=\rho_{t}\left(-\rho_{s}\left(\xi_{T}\right)\right)
$$

which shows the property of time consistency and maturity independence.

Fourthly, using the BSDE representation of the forward entropic risk measures, we can obtain their convex dual representation as well:

$$
\rho_{t}\left(\xi_{T}\right)=-\underset{q \in \mathcal{A}_{[t, T]}^{*}}{\operatorname{essinf}} E_{\mathbb{Q}^{q}}\left[\xi_{T}+\int_{t}^{T} G^{*}\left(V_{s}, Z_{s}, q_{s}\right) d s \mid \mathcal{F}_{t}\right],
$$

where $\mathcal{A}_{[0, T]}^{*}$ is the admissible set:

$$
\mathcal{A}_{[0, T]}^{*} \triangleq\left\{q \in \mathcal{L}_{B M O}^{2}[0, T]: E_{\mathbb{Q}^{q}}\left[\int_{0}^{T}\left|G^{*}\left(V_{s}, Z_{s}, q_{s}\right)\right| d s\right]<\infty\right\},
$$


for any $q \in \mathcal{L}_{B M O}^{2}[0, T], \mathbb{Q}^{q}$ is a probability measure on $\mathcal{F}_{T}$ defined by

$$
\frac{d \mathbb{Q}^{q}}{d \mathbb{P}}=\mathcal{E}\left(\int_{0}^{\cdot} q_{s}^{t r} d W_{s}\right)_{T},
$$

and $G^{*}: \mathbb{R}^{d} \times \mathbb{R}^{d} \times \mathbb{R}^{d} \rightarrow \mathbb{R} \cup\{\infty\}$ is the convex dual of the convex function $G$ in 3.5 .12 in $\hat{z}$. Using this convex dual representation of forward entropic risk measures, we can show their properties of anti-positivity, translation invariance, and convexity as well.

Finally, by the indifference condition 3.2 .2 to define the forward entropic risk measures, we can also define the hedging strategies associated with the risk positions as the difference of the optimal strategies for the two optimization problems in (3.2.2), as in the classical framework. In other words, the hedging strategy $\alpha_{\cdot, T}=\left\{\alpha_{t, T}\right\}_{t \in[0, T]}$ for the forward entropic risk measure is given by

$$
\begin{aligned}
\alpha_{t, T} & =\pi_{t}^{*, \xi}-\pi_{t}^{*} \\
& =\mathcal{P}_{\Pi}\left(Z_{t}^{T, \xi}+\frac{Z_{t}+\theta\left(V_{t}\right)}{\gamma}\right)-\mathcal{P}_{\Pi}\left(\frac{Z_{t}+\theta\left(V_{t}\right)}{\gamma}\right), \quad \forall t \in[0, T] .
\end{aligned}
$$

Note that the first term naturally depends on the maturity of the risk position, while the second one is independent of it and is defined for all times. On the other hand, in the classical framework, both terms depend on the investment horizon.

Taking the advantage of the BSDE representations for the forward entropic risk measures, we shall study the large maturity behavior of them.

\subsection{Large Maturity Behavior of Forward Entropic Risk Measures}

This section provides another main result of this chapter, which is the large maturity behavior of the forward entropic risk measures on the European type risk positions with respect to the stochastic factors. Mathematically, define, for any 
$t \geq 0$,

$$
\begin{aligned}
\mathcal{L}_{M}^{\infty}\left(\mathcal{F}_{t}\right) \triangleq\left\{\xi_{t} \in \mathcal{L}^{\infty}\left(\mathcal{F}_{t}\right):\right. & \xi_{t}=-g\left(V_{t}\right), \text { for some function } g: \mathbb{R}^{d} \rightarrow \mathbb{R} \text { being } \\
& \text { uniformly bounded and Lipschitz continuous with a } \\
& \text { Lipschitz constant } \left.C_{g}\right\}
\end{aligned}
$$

Moreover, define

$$
\mathcal{L}_{M} \triangleq \cup_{t \geq 0} \mathcal{L}_{M}^{\infty}\left(\mathcal{F}_{t}\right)
$$

In the remaining of this chapter, we shall study the forward entropic risk measures on the risk positions $\xi \in \mathcal{L}_{M}$.

By Theorem 3.5.1, for any $T \geq 0$ and $\xi_{T} \in \mathcal{L}_{M}^{\infty}\left(\mathcal{F}_{T}\right)$,

$$
\rho_{t}\left(\xi_{T}\right)=Y_{t}^{T, g}, \quad \forall t \in[0, T]
$$

where $\left(Y^{T, g}=\left\{Y_{t}^{T, g}\right\}_{t \in[0, T]}, Z^{T, g}=\left\{Z_{t}^{T, g}\right\}_{t \in[0, T]}\right)$ is the solution of the finite horizon BSDE (3.5.14):

$$
Y_{t}^{T, g}=g\left(V_{T}\right)+\int_{t}^{T} G\left(V_{s}, Z_{s}, Z_{s}^{T, g}\right) d s-\int_{t}^{T}\left(Z_{s}^{T, g}\right)^{t r} d W_{s}, \quad \forall t \in[0, T] .
$$

Thanks to the BSDE representation, the large maturity behavior of the forward entropic risk measure is then given by that of the solution $Y^{T, g}$ of the finite horizon BSDE (3.6.17). Hence, it is natural to relate the finite horizon BSDE (3.6.17) with an ergodic BSDE:

$$
\hat{Y}_{t}=\hat{Y}_{T^{\prime}}+\int_{t}^{T^{\prime}}\left(G\left(V_{s}, Z_{s}, \hat{Z}_{s}\right)-\hat{\lambda}\right) d s-\int_{t}^{T^{\prime}}\left(\hat{Z}_{s}\right)^{t r} d W_{s}, \quad \forall T^{\prime} \geq 0, t \in[0, T],
$$

where its solution triplet, if exist, is denoted by $\left(\hat{Y}=\left\{\hat{Y}_{t}\right\}_{t \geq 0}, \hat{Z}=\left\{\hat{Z}_{t}\right\}_{t \geq 0}, \hat{\lambda}\right)$. We should then examine the proximity of the solutions $\left(Y^{T, g}, Z^{T, g}\right)$ and $(\hat{Y}, \hat{Z}, \hat{\lambda})$. However, the driver $G$ of the ergodic BSDE (3.6.18) depends on the solution $Z$ of the ergodic BSDE (3.4.8) representing the exponential forward investment performance process. This leads us to study the large maturity behavior of the 
forward entropic risk measure by the Markovian and non-Markovian exponential forward investment performance processes separately.

\subsubsection{Markovian Exponential Forward Investment Perfor- mance Processes}

In this subsection, we consider the case that the exponential forward investment performance process is in stochastic factor form (3.4.11):

$$
U(x, t)=-e^{-\gamma x+y\left(V_{t}\right)-\lambda t}, \quad \forall x \in \mathbb{R}, t \geq 0,
$$

with the solution triplet $(y, z, \lambda)$ of the ergodic BSDE (3.4.8) given in Proposition 3.4.1. For the ease of later discussions, with the Markovian solution triplet $(y, z, \lambda)$ of the ergodic BSDE (3.4.8), we rewrite respectively the finite horizon BSDE 3.6.17) and the ergodic BSDE (3.6.18):

$$
Y_{t}^{T, g}=g\left(V_{T}\right)+\int_{t}^{T} G\left(V_{s}, z\left(V_{s}\right), Z_{s}^{T, g}\right) d s-\int_{t}^{T}\left(Z_{s}^{T, g}\right)^{t r} d W_{s}, \quad \forall t \in[0, T],
$$

and

$\hat{Y}_{t}=\hat{Y}_{T^{\prime}}+\int_{t}^{T^{\prime}}\left(G\left(V_{s}, z\left(V_{s}\right), \hat{Z}_{s}\right)-\hat{\lambda}\right) d s-\int_{t}^{T^{\prime}}\left(\hat{Z}_{s}\right)^{t r} d W_{s}, \quad \forall T^{\prime} \geq 0, t \in\left[0, T^{\prime}\right]$.

In this case, the driver $G$ of the ergodic BSDE (3.6.21) depends on the function $z$. Therefore, although, by Proposition 3.4.1, that the function $z$ is uniformly bounded, the function $G$ satisfies the locally Lipschitz estimate 3.4 .7 in $\hat{z}$ by 3.5.13, it may not satisfy the locally Lipschitz estimate 3.4.6 in $v$. Hence, the existence and uniqueness results in Proposition 3.4.1 by Liang and Zariphopoulou (2017) might not apply to the ergodic BSDE 3.6.21.

To remedy this issue, we first study an auxiliary finite horizon BSDE:

$\hat{Y}_{t}^{T, g}=g\left(V_{T}\right)+\frac{y\left(V_{T}\right)-\lambda T}{\gamma}+\int_{t}^{T} \frac{1}{\gamma} F\left(V_{s}, \gamma \hat{Z}_{s}^{T, g}\right) d s-\int_{t}^{T}\left(\hat{Z}_{s}^{T, g}\right)^{t r} d W_{s}, \quad \forall t \in[0, T]$, 
where its solution pair, if exist, is denoted by $\left(\hat{Y}^{T, g}=\left\{\hat{Y}_{t}^{T, g}\right\}_{t \in[0, T]}, \hat{Z}^{T, g}=\right.$ $\left.\left\{\hat{Z}_{t}^{T, g}\right\}_{t \in[0, T]}\right)$. Moreover, any solution pair $\left(\hat{Y}^{T, g}, \hat{Z}^{T, g}\right)$, which solves the finite horizon BSDE (3.6.22), is Markovian with respect to the stochastic factors. In other words, for any solution pair $\left(\hat{Y}^{T, g}, \hat{Z}^{T, g}\right)$ of the finite horizon BSDE 3.6.22, there exist functions $\hat{y}^{T, g}: \mathbb{R}^{d} \times[0, T] \rightarrow \mathbb{R}$ and $\hat{z}^{T, g}: \mathbb{R}^{d} \times[0, T] \rightarrow \mathbb{R}^{d}$ such that

$$
\hat{Y}_{t}^{T, g}=\hat{y}^{T, g}\left(V_{t}, t\right), \quad \forall t \in[0, T],
$$

and

$$
\hat{Z}_{t}^{T, g}=\hat{z}^{T, g}\left(V_{t}, t\right), \quad \forall t \in[0, T] .
$$

We shall show that the finite horizon BSDE (3.6.22) admits a solution pair such that the function $\hat{z}^{T, g}$ is uniformly bounded. We start with a property of the stochastic factor processes.

Proposition 3.6.1. Suppose that Assumption 3.3.2 holds. Then,

$$
\left|V_{s}^{t, v}-V_{s}^{t, \bar{v}}\right|^{2} \leq e^{-2 C_{\eta}(s-t)}|v-\bar{v}|^{2}, \quad \forall v, \bar{v} \in \mathbb{R}^{d}, 0 \leq t \leq s<\infty .
$$

Proof. The proof follows from the arguments of Grönwall's inequality and the application of the dissipative condition (3.3.3) for the stochastic factors.

Lemma 3.6.2. Suppose that Assumptions 3.3 .1 and 3.3.2 hold, and that the exponential forward investment performance process is given by (3.6.19). Then there exists a Markovian solution $\left(\hat{Y}^{T, g}, \hat{Z}^{T, g}\right)$ of the finite horizon BSDE (3.6.22), with the functions $\hat{y}^{T, g}: \mathbb{R}^{d} \times[0, T] \rightarrow \mathbb{R}$ and $\hat{z}^{T, g}: \mathbb{R}^{d} \times[0, T] \rightarrow \mathbb{R}^{d}$ such that

$$
\begin{aligned}
& \hat{Y}_{t}^{T, g}=\hat{y}^{T, g}\left(V_{t}, t\right), \quad \forall t \in[0, T], \\
& \hat{Z}_{t}^{T, g}=\hat{z}^{T, g}\left(V_{t}, t\right), \quad \forall t \in[0, T],
\end{aligned}
$$

and the function $\hat{z}^{T, g}$ is uniformly bounded:

$$
\left|\hat{z}^{T, g}(v, t)\right| \leq q \triangleq \frac{\gamma C_{\eta} C_{g}+C_{v}}{\gamma\left(C_{\eta}-C_{v}\right)}+\frac{C_{\eta} C_{v}}{\gamma\left(C_{\eta}-C_{v}\right)^{2}}, \quad \forall v \in \mathbb{R}^{d}, t \in[0, T] .
$$


Proof. Let $t \in[0, T]$ and $v, \bar{v} \in \mathbb{R}^{d}$. Consider the finite horizon BSDE:

$$
\begin{array}{r}
\hat{Y}_{s}^{T, g, t, v}=g\left(V_{T}^{t, v}\right)+\frac{y\left(V_{T}^{t, v}\right)-\lambda T}{\gamma}+\int_{s}^{T} \frac{1}{\gamma} F\left(V_{u}^{t, v}, \gamma \hat{Z}_{u}^{T, g, t, v}\right) d u-\int_{s}^{T}\left(\hat{Z}_{u}^{T, g, t, v}\right)^{t r} d W_{u}, \\
\forall s \in[t, T],
\end{array}
$$

which is simply the finite horizon BSDE (3.6.22) by denoting the dependence of the initial condition $v \in \mathbb{R}^{d}$ of the stochastic factors at the initial time $t \in[0, T]$. Define a function $q: \mathbb{R}^{d} \rightarrow \mathbb{R}^{d}$ by

$$
q(z)=\frac{\min (|z|, q)}{|z|} z \mathbb{1}_{\{z \neq 0\}}, \quad \forall z \in \mathbb{R}^{d} .
$$

Consider a truncated version of the finite horizon BSDE (3.6.23):

$$
\begin{array}{r}
\hat{Y}_{s}^{T, g, t, v}=g\left(V_{T}^{t, v}\right)+\frac{y\left(V_{T}^{t, v}\right)-\lambda T}{\gamma}+\int_{s}^{T} \frac{1}{\gamma} F\left(V_{u}^{t, v}, \gamma q\left(\hat{Z}_{u}^{T, g, t, v}\right)\right) d u-\int_{s}^{T}\left(\hat{Z}_{u}^{T, g, t, v}\right)^{t r} d W_{u}, \\
\forall s \in[t, T] .
\end{array}
$$

By (3.4.6), 3.4.7) and (3.6.24), for any $v_{1}, v_{2}, z_{1}, z_{2} \in \mathbb{R}^{d}$,

$$
\left|F\left(v_{1}, \gamma q\left(z_{1}\right)\right)-F\left(v_{2}, \gamma q\left(z_{1}\right)\right)\right| \leq C_{v}(1+\gamma q)\left|v_{1}-v_{2}\right|,
$$

and

$$
\left|F\left(v_{1}, \gamma q\left(z_{1}\right)\right)-F\left(v_{1}, \gamma q\left(z_{2}\right)\right)\right| \leq C_{z}(1+2 \gamma q) \gamma\left|z_{1}-z_{2}\right| .
$$

Moreover, by the uniform boundedness of the function $g$ and the linear growth of the function $y$, we deduce that the terminal condition of the finite horizon BSDE (3.6.25) is square-integrable:

$$
\mathbb{E}\left[\left|g\left(V_{T}^{t, v}\right)+\frac{y\left(V_{T}^{t, v}\right)-\lambda T}{\gamma}\right|^{2}\right] \leq C\left(1+\mathbb{E}\left[\left|V_{T}^{t, v}\right|^{2}\right]\right)<\infty .
$$

Hence, by Pardoux and Peng (1990), there exists a unique Markovian solution $\left(\hat{Y}^{T, g, t, v}=\left\{\hat{Y}_{s}^{T, g, t, v}\right\}_{s \in[t, T]}, \hat{Z}^{T, g, t, v}=\left\{\hat{Z}_{s}^{T, g, t, v}\right\}_{s \in[t, T]}\right)$ of the finite horizon BSDE (3.6.25), with

$$
\hat{Y}_{s}^{T, g, t, v}=\hat{y}^{T, g}\left(V_{s}^{t, v}, s\right), \quad \forall s \in[t, T],
$$


and

$$
\hat{Z}_{s}^{T, g, t, v}=\hat{z}^{T, g}\left(V_{s}^{t, v}, s\right), \quad \forall s \in[t, T]
$$

By telescoping terms,

$$
\begin{aligned}
\hat{Y}_{t}^{T, g, t, v}-\hat{Y}_{t}^{T, g, t, \bar{v}}= & g\left(V_{T}^{t, v}\right)-g\left(V_{T}^{t, \bar{v}}\right)+\frac{1}{\gamma}\left(y\left(V_{T}^{t, v}\right)-y\left(V_{T}^{t, \bar{v}}\right)\right) \\
& +\int_{t}^{T} \frac{1}{\gamma}\left(F\left(V_{s}^{t, v}, \gamma q\left(\hat{Z}_{s}^{T, g, t, v}\right)\right)-F\left(V_{s}^{t, \bar{v}}, \gamma q\left(\hat{Z}_{s}^{T, g, t, v}\right)\right)\right) d s \\
& +\int_{t}^{T} \frac{1}{\gamma}\left(F\left(V_{s}^{t, \bar{v}}, \gamma q\left(\hat{Z}_{s}^{T, g, t, v}\right)\right)-F\left(V_{s}^{t, \bar{v}}, \gamma q\left(\hat{Z}_{s}^{T, g, t, \bar{v}}\right)\right)\right) d s \\
& -\int_{t}^{T}\left(\hat{Z}_{s}^{T, g, t, v}-\hat{Z}_{s}^{T, g, t, \bar{v}}\right)^{t r} d W_{s} .
\end{aligned}
$$

Define a process $M=\left\{M_{s}\right\}_{s \in[t, T]}$ by

$$
\begin{gathered}
M_{s}=\frac{\left(F\left(V_{s}^{t, \bar{v}}, \gamma q\left(\hat{Z}_{s}^{T, g, t, v}\right)\right)-F\left(V_{s}^{t, \bar{v}}, \gamma q\left(\hat{Z}_{s}^{T, g, t, \bar{v}}\right)\right)\right)\left(\hat{Z}_{s}^{T, g, t, v}-\hat{Z}_{s}^{T, g, t, \bar{v}}\right)}{\gamma\left|\hat{Z}_{s}^{T, g, t, v}-\hat{Z}_{s}^{T, g, t, \bar{v}}\right|^{2}} \mathbb{1}_{\left\{\hat{Z}_{s}^{T, g, t, v}-\hat{Z}_{s}^{T, g, t, \bar{v}} \neq 0\right\}}, \\
\forall s \in[t, T] .
\end{gathered}
$$

By (3.6.27), the process $M$ is uniformly bounded. By Girsanov Theorem, there exists a $\mathbb{P}$-equivalent probability measure $\mathbb{Q}^{M}$ defined on $\mathcal{F}_{T}$ such that the process $W^{M}=\left\{W_{s}^{M}\right\}_{s \in[0, T]}$, which is defined by

$$
W_{s}^{M}=W_{s}-\int_{0}^{s} M_{u} d u, \quad \forall s \in[0, T]
$$

is a $\mathbb{Q}^{M}$-Brownian motion. Therefore,

$$
\begin{aligned}
\hat{Y}_{t}^{T, g, t, v}-\hat{Y}_{t}^{T, g, t, \bar{v}}= & g\left(V_{T}^{t, v}\right)-g\left(V_{T}^{t, \bar{v}}\right)+\frac{1}{\gamma}\left(y\left(V_{T}^{t, v}\right)-y\left(V_{T}^{t, \bar{v}}\right)\right) \\
& +\int_{t}^{T} \frac{1}{\gamma}\left(F\left(V_{s}^{t, v}, \gamma q\left(\hat{Z}_{s}^{T, g, t, v}\right)\right)-F\left(V_{s}^{t, \bar{v}}, \gamma q\left(\hat{Z}_{s}^{T, g, t, v}\right)\right)\right) d s \\
& -\int_{t}^{T}\left(\hat{Z}_{s}^{T, g, t, v}-\hat{Z}_{s}^{T, g, t, \bar{v}}\right)^{t r} d W_{s}^{M} .
\end{aligned}
$$

Taking the conditional $\mathbb{Q}^{M}$-expectation with respect to $\mathcal{F}_{t}$, by the Lipschitz con- 
tinuous property of $g,(3.4 .9)$, and (3.6.26),

$$
\begin{aligned}
\left|\hat{Y}_{t}^{T, g, t, v}-\hat{Y}_{t}^{T, g, t, \bar{v}}\right| \leq & C_{g} \mathbb{E}^{\mathbb{Q}^{M}}\left[\left|V_{T}^{t, v}-V_{T}^{t, \bar{v}}\right| \mid \mathcal{F}_{t}\right]+\frac{C_{v}}{\gamma\left(C_{\eta}-C_{v}\right)} \mathbb{E}^{\mathbb{Q}^{M}}\left[\mid V_{T}^{t, v}-V_{T}^{t, \bar{v}} \| \mathcal{F}_{t}\right] \\
& +\frac{C_{v}(1+\gamma q)}{\gamma} \mathbb{E}\left[\int_{t}^{T}\left|V_{s}^{t, v}-V_{s}^{t, \bar{v}}\right| d s \mid \mathcal{F}_{t}\right] .
\end{aligned}
$$

By Proposition 3.6.1,

$$
\left|\hat{Y}_{t}^{T, g, t, v}-\hat{Y}_{t}^{T, g, t, \bar{v}}\right| \leq\left(C_{g}+\frac{C_{v}}{\gamma\left(C_{\eta}-C_{v}\right)}+\frac{C_{v}(1+\gamma q)}{\gamma C_{\eta}}\right)|v-\bar{v}| .
$$

Hence,

$$
\left|\nabla y^{T, g}(v, t)\right| \leq C_{g}+\frac{C_{v}}{\gamma\left(C_{\eta}-C_{v}\right)}+\frac{C_{v}(1+\gamma q)}{\gamma C_{\eta}}=q
$$

Finally, using the relationship that

$$
\kappa^{t r} \nabla \hat{Y}_{s}^{T, g, t, v}=\hat{Z}_{s}^{T, g, t, v}, \quad \forall s \in[t, T]
$$

in particular,

$$
\kappa^{t r} \nabla \hat{y}^{T, g}(v, t)=\hat{z}^{T, g}(v, t)
$$

we have, by $|\kappa|=1$,

$$
\left|\hat{z}^{T, g}(v, t)\right| \leq q
$$

Therefore, by the definition of the function $q$ in 3.6.24),

$$
q\left(\hat{Z}_{s}^{T, g, t, v}\right)=\hat{Z}_{s}^{T, g, t, v}, \quad \forall s \in[t, T]
$$

and hence the unique Markovian solution $\left(\hat{Y}^{T, g, t, v}, \hat{Z}^{T, g, t, v}\right)$ of the finite horizon BSDE (3.6.25) also solves the finite horizon BSDE (3.6.23). In particular, the function $\hat{z}^{T, g}$ is uniformly bounded.

Define a pair of processes $\left(Y^{T, g}=\left\{Y_{t}^{T, g}\right\}_{t \in[0, T]}, Z^{T, g}=\left\{Z_{t}^{T, g}\right\}_{t \in[0, T]}\right)$ by

$$
\begin{aligned}
Y_{t}^{T, g} & \triangleq \hat{Y}_{t}^{T, g}-\frac{Y_{t}-\lambda t}{\gamma} \\
& =\hat{y}^{T, g}\left(V_{t}, t\right)-\frac{y\left(V_{t}\right)-\lambda t}{\gamma} \\
& =y^{T, g}\left(V_{t}, t\right), \quad \forall t \in[0, T],
\end{aligned}
$$


and

$$
\begin{aligned}
Z_{t}^{T, g} & \triangleq \hat{Z}_{t}^{T, g}-\frac{Z_{t}}{\gamma} \\
& =\hat{z}^{T, g}\left(V_{t}, t\right)-\frac{z\left(V_{t}\right)}{\gamma} \\
& =z^{T, g}\left(V_{t}, t\right), \quad \forall t \in[0, T],
\end{aligned}
$$

where $\left(\hat{Y}^{T, g}, \hat{Z}^{T, g}\right)$ is the Markovian solution of the finite horizon BSDE 3.6.22 in Lemma 3.6.2, and for some functions $y^{T, g}: \mathbb{R}^{d} \times[0, T] \rightarrow \mathbb{R}$ and $z^{T, g}: \mathbb{R}^{d} \times$ $[0, T] \rightarrow \mathbb{R}^{d}$. Then, by the ergodic BSDE (3.4.8) on the interval $[0, T]$, the pair of processes $\left(Y^{T, g}, Z^{T, g}\right)$, defined by 3.6 .28 and 3.6 .29 , solve the finite horizon BSDE 3.6.20. We shall show that the solution $\left(Y^{T, g}, Z^{T, g}\right)$ of the finite horizon BSDE (3.6.20), defined by 3.6.28) and 3.6.29), is actually the unique Markovian solution of the finite horizon BSDE (3.6.20) as depicted by the statement (i) in Theorem 3.5.1. We begin with other properties of the stochastic factor processes.

Proposition 3.6.3. Suppose that Assumption 3.3.2 holds, and that the stochastic factor process $V$ satisfies:

$$
d V_{t}^{v}=\left(\eta\left(V_{t}^{v}\right)+H\left(V_{t}^{v}\right)\right) d t+\kappa d W_{t}^{H}, \quad \forall t \geq 0
$$

for some bounded measurable function $H: \mathbb{R}^{d} \rightarrow \mathbb{R}^{d}, \mathbb{P}$-equivalent probability measure $\mathbb{Q}^{H}$, and $\mathbb{Q}^{H}$-Brownian motion $W^{H}=\left\{W_{t}^{H}\right\}_{t \geq 0}$. Then,

(i) there exists a constant $C>0$ such that, for any $v \in \mathbb{R}^{d}$,

$$
\mathbb{E}^{\mathbb{Q}^{H}}\left[\left|V_{t}^{v}\right|^{2}\right] \leq C\left(1+|v|^{2}\right), \quad \forall t \geq 0
$$

(ii) for any measurable function $\phi: \mathbb{R}^{d} \rightarrow \mathbb{R}$ with polynomial growth rate $\mu>0$, and $v, \bar{v} \in \mathbb{R}^{d}$,

$$
\left|\mathbb{E}^{\mathbb{Q}^{H}}\left[\phi\left(V_{t}^{v}\right)-\phi\left(V_{t}^{\bar{v}}\right)\right]\right| \leq C\left(1+|v|^{1+\mu}+|\bar{v}|^{1+\mu}\right) e^{-\hat{C}_{\eta} t}, \quad \forall t \geq 0,
$$

where the constants $C$ and $\hat{C}_{\eta}$ depend on the function $H$ only through $\sup _{v \in \mathbb{R}^{d}}|H(v)|$. 
Proof. For the proof of (i), see Lemma 3.1 in Fleming and McEneaney (1995), while for the proof of (ii), see Lemma 3.4 in $\mathrm{Hu}$ et al. (2015).

Lemma 3.6.4. Suppose that Assumptions 3.3.1 and 3.3.2 hold, and that the exponential forward investment performance process is given by (3.6.19). Then the pair of processes $\left(Y^{T, g}, Z^{T, g}\right)$, defined by (3.6.28) and (3.6.29), is the unique Markovian solution of the finite horizon BSDE (3.6.20) such that $Y^{T, g}$ is uniformly bounded and $Z^{T, g} \in \mathcal{L}_{B M O}^{2}[0, T]$. Moreover,

(i) there exists a constant $C>0$ such that

$$
\left|y^{T, g}(v, t)\right| \leq C(1+|v|), \quad \forall v \in \mathbb{R}^{d}, t \in[0, T]
$$

(ii)

$$
\left|\nabla y^{T, g}(v, t)\right| \leq q+\frac{C_{v}}{\gamma\left(C_{\eta}-C_{v}\right)}, \quad \forall v \in \mathbb{R}^{d}, t \in[0, T]
$$

(iii)

$$
\left|y^{T, g}(v, t)-y^{T, g}(\bar{v}, t)\right| \leq C\left(1+|v|^{2}+|\bar{v}|^{2}\right) e^{-\hat{C}_{\eta}(T-t)}, \quad \forall v, \bar{v} \in \mathbb{R}^{d}, t \in[0, T]
$$

Proof. We have showed that $\left(Y^{T, g}, Z^{T, g}\right)$, defined by 3.6 .28 and 3.6 .29 , solve the finite horizon BSDE (3.6.20).

By the definition of $Z^{T, g}$ in 3.6 .29 , the uniform boundedness of the function $\hat{z}^{T, g}$ by Lemma 3.6 .2 , and the uniform boundedness of the function $z$ by Proposition 3.4.1,

$$
\begin{aligned}
\left|Z_{t}^{T, g}\right| & =\left|\hat{z}^{T, g}\left(V_{t}, t\right)-\frac{z\left(V_{t}\right)}{\gamma}\right| \\
& \leq q+\frac{C_{v}}{\gamma\left(C_{\eta}-C_{v}\right)}, \quad \forall t \in[0, T] .
\end{aligned}
$$

Then, the uniform boundedness of $Z^{T, g}$ obviously implies that $Z^{T, g} \in \mathcal{L}_{B M O}^{2}[0, T]$. 
Since $\left(Y^{T, g}, Z^{T, g}\right)$ solve the finite horizon BSDE 3.6.20,

$$
\begin{array}{r}
Y_{t}^{T, g}=g\left(V_{T}\right)+\int_{t}^{T} \frac{1}{\gamma}\left(F\left(V_{s}, z\left(V_{s}\right)+\gamma Z_{s}^{T, g}\right)-F\left(V_{s}, z\left(V_{s}\right)\right)\right) d s-\int_{t}^{T}\left(Z_{s}^{T, g}\right)^{t r} d W_{s}, \\
\forall t \in[0, T] .
\end{array}
$$

Define a process $H=\left\{H\left(V_{s}\right)\right\}_{s \in[0, T]}$ by

$$
H\left(V_{s}\right)=\frac{\left(F\left(V_{s}, z\left(V_{s}\right)+\gamma Z_{s}^{T, g}\right)-F\left(V_{s}, z\left(V_{s}\right)\right)\right) Z_{s}^{T, g}}{\gamma\left|Z_{s}^{T, g}\right|^{2}} \mathbb{1}_{\left\{Z_{s}^{T, g} \neq 0\right\}}, \quad \forall s \in[0, T] .
$$

By (3.4.7), the uniform boundedness of the function $z$ by Proposition 3.4.1, and the uniform boundedness of $Z^{T, g}$, the process $H$ is uniformly bounded. By Girsanov Theorem, there exists a $\mathbb{P}$-equivalent probability measure $\mathbb{Q}^{H}$ defined on $\mathcal{F}_{T}$ such that the process $W^{H}=\left\{W_{s}^{H}\right\}_{s \in[0, T]}$, which is defined by

$$
W_{s}^{H}=W_{s}-\int_{0}^{s} H\left(V_{u}\right) d u, \quad \forall s \in[0, T]
$$

is a $\mathbb{Q}^{H}$-Brownian motion. Therefore,

$$
Y_{t}^{T, g}=g\left(V_{T}\right)-\int_{t}^{T}\left(Z_{s}^{T, g}\right)^{t r} d W_{s}^{H}, \quad \forall t \in[0, T] .
$$

Taking the conditional $\mathbb{Q}^{H}$-expectation with respect to $\mathcal{F}_{t}$,

$$
Y_{t}^{T, g}=\mathbb{E}^{\mathbb{Q}^{H}}\left[g\left(V_{T}\right) \mid \mathcal{F}_{t}\right], \quad \forall t \in[0, T]
$$

Therefore, by the uniform boundedness of the function $g$, there exists a constant $C>0$ such that

$$
\left|Y_{t}^{T, g}\right| \leq C, \quad \forall t \in[0, T]
$$

Hence, we showed that $\left(Y^{T, g}, Z^{T, g}\right)$, defined by 3.6 .28 and 3.6 .29 , solve the finite horizon BSDE (3.6.20), such that $Y^{T, g}$ is uniformly bounded and $Z^{T, g} \in$ $\mathcal{L}_{B M O}^{2}[0, T]$. By the statement (i) in Theorem 3.5.1. $\left(Y^{T, g}, Z^{T, g}\right)$ is the unique Markovian solution of the finite horizon BSDE 3.6.20 such that $Y^{T, g}$ is uniformly bounded and $Z^{T, g} \in \mathcal{L}_{B M O}^{2}[0, T]$. 
Let $t \in[0, T]$ and $v, \bar{v} \in \mathbb{R}^{d}$. Since $\left(Y^{T, g}, Z^{T, g}\right)$ solves the finite horizon BSDE (3.6.20,

$$
\begin{aligned}
Y_{t}^{T, g, t, v}= & g\left(V_{T}^{t, v}\right)+\int_{t}^{T} \frac{1}{\gamma}\left(F\left(V_{s}^{t, v}, z\left(V_{s}^{t, v}\right)+\gamma Z_{s}^{T, g, t, v}\right)-F\left(V_{s}^{t, v}, z\left(V_{s}^{t, v}\right)\right)\right) d s \\
& -\int_{t}^{T}\left(Z_{s}^{T, g, t, v}\right)^{t r} d W_{s} .
\end{aligned}
$$

(ii) By the definition of $Z^{T, g, t, v}$ in 3.6 .29 , the uniform boundedness of the function $\hat{z}^{T, g}$ by Lemma 3.6.2, and the uniform boundedness of the function $z$ by Proposition 3.4 .1 ,

$$
\begin{aligned}
\left|Z_{s}^{T, g, t, v}\right| & =\left|\hat{z}^{T, g}\left(V_{s}^{t, v}, s\right)-\frac{z\left(V_{s}^{t, v}\right)}{\gamma}\right| \\
& \leq q+\frac{C_{v}}{\gamma\left(C_{\eta}-C_{v}\right)}, \quad \forall s \in[t, T] .
\end{aligned}
$$

Using the relationship that

$$
\kappa^{t r} \nabla Y_{s}^{T, g, t, v}=Z_{s}^{T, g, t, v}, \quad \forall s \in[t, T]
$$

in particular,

$$
\kappa^{t r} \nabla y^{T, g}(v, t)=z^{T, g}(v, t),
$$

we have, by $|\kappa|=1$,

$$
\left|\nabla y^{T, g}(v, t)\right| \leq q+\frac{C_{v}}{\gamma\left(C_{\eta}-C_{v}\right)}
$$

(i) Define a process $H^{t, v}=\left\{H\left(V_{s}^{t, v}\right)\right\}_{s \in[t, T]}$ by

$$
\begin{array}{r}
H\left(V_{s}^{t, v}\right)=\frac{\left(F\left(V_{s}^{t, v}, z\left(V_{s}^{t, v}\right)+\gamma Z_{s}^{T, g, t, v}\right)-F\left(V_{s}^{t, v}, z\left(V_{s}^{t, v}\right)\right)\right) Z_{s}^{T, g, t, v}}{\gamma\left|Z_{s}^{T, g, t, v}\right|^{2}} \mathbb{1}_{\left\{Z_{s}^{T, g, t, v} \neq 0\right\}}, \\
\forall s \in[t, T] .
\end{array}
$$

By (3.4.7), the uniform boundedness of the function $z$ by Proposition 3.4.1, and the uniform boundedness of $Z^{T, g, t, v}$, the process $H^{t, v}$ is uniformly bounded. By Girsanov Theorem, there exists a $\mathbb{P}$-equivalent probability measure $\mathbb{Q}^{H^{t, v}}$ 
defined on $\mathcal{F}_{T}$ such that the process $W^{H}=\left\{W_{s}^{H^{t, v}}\right\}_{s \in[0, T]}$, which is defined by

$$
W_{s}^{H^{t, v}}=W_{s}-\int_{0}^{s} H\left(V_{u}^{t, v}\right) d u, \quad \forall s \in[0, T],
$$

is a $\mathbb{Q}^{H^{t, v}}$-Brownian motion. Therefore,

$$
Y_{t}^{T, g, t, v}=g\left(V_{T}^{t, v}\right)-\int_{t}^{T}\left(Z_{s}^{T, g, t, v}\right)^{t r} d W_{s}^{H^{t, v}} .
$$

Taking the conditional $\mathbb{Q}^{H^{t, v}}$-expectation with respect to $\mathcal{F}_{t}$,

$$
Y_{t}^{T, g, t, v}=\mathbb{E}^{\mathbb{Q}^{H^{t, v}}}\left[g\left(V_{T}^{t, v}\right) \mid \mathcal{F}_{t}\right]
$$

By the uniform bounded and Lipschitz continuous property, and thus the linear growth property, of the function $g$, and the statement (i) in Proposition 3.6 .3 ,

$$
\begin{aligned}
\left|Y_{t}^{T, g, t, v}\right|^{2} & \leq \mathbb{E}^{\mathbb{Q}^{H^{t, v}}}\left[\left|g\left(V_{T}^{t, v}\right)\right|^{2} \mid \mathcal{F}_{t}\right] \\
& \leq \mathbb{E}^{\mathbb{Q}^{H^{t, v}}}\left[\left|C\left(1+\left|V_{T}^{t, v}\right|\right)\right|^{2} \mid \mathcal{F}_{t}\right] \\
& \leq 2 C^{2}+2 C^{2} \mathbb{E}^{\mathbb{Q}^{H^{t, v}}}\left[\left|V_{T}^{t, v}\right|^{2} \mid \mathcal{F}_{t}\right] \\
& \leq 2 C^{2}+2 C^{2} \times C_{1}\left(1+|v|^{2}\right) \\
& \leq \tilde{C}(1+|v|)^{2} .
\end{aligned}
$$

Hence,

$$
\left|y^{T, g}(v, t)\right| \leq \sqrt{\tilde{C}}(1+|v|) .
$$

(iii) By 3.6.30 and the statement (ii) in Proposition 3.6.3.

$$
\begin{aligned}
\left|y^{T, g}(v, t)-y^{T, g}(\bar{v}, t)\right| & =\left|\mathbb{E}^{\mathbb{Q}^{H^{t, v}}}\left[g\left(V_{T}^{t, v}\right)-g\left(V_{T}^{t, \bar{v}}\right) \mid \mathcal{F}_{t}\right]\right| \\
& =\left|\mathbb{E}^{\mathbb{Q}^{H^{t, v}}}\left[g\left(V_{T-t}^{0, v}\right)-g\left(V_{T-t}^{0, \bar{v}}\right)\right]\right| \\
& \leq C\left(1+|v|^{2}+|\bar{v}|^{2}\right) e^{-\hat{C}_{\eta}(T-t)} .
\end{aligned}
$$


Finally, we are ready for the second main result of this chapter with the Markovian exponential forward investment performance processes for risk positions in $\mathcal{L}_{M}$. We shall show that as the maturity $T$ tends to infinity, the forward entropic risk measure tends to a constant, which is independent of the initial state of the stochastic factor process. We shall also provide the rate of convergence. Since $T$ shall be tending to infinity, the result shall be stated and proved at the time $t=0$ only.

Theorem 3.6.5. Suppose that Assumptions 3.3.1 and 3.3.2 hold, and that the exponential forward investment performance process is given by 3.6.19. Let $T \geq$ 0 and $\xi_{T} \in \mathcal{L}_{M}$. Then,

(i) there exists a constant $C>0$ such that

$$
\frac{\left|\rho_{0}\left(\xi_{T}\right)\right|}{T} \leq \frac{C(1+|v|)}{T}, \quad \forall v \in \mathbb{R}^{d}
$$

and hence,

$$
\lim _{T \uparrow \infty} \frac{\rho_{0}\left(\xi_{T}\right)}{T}=0
$$

(ii) there exists a constant $L^{g} \in \mathbb{R}$, independent of $V_{0}^{v}=v$, such that

$$
\lim _{T \uparrow \infty} \rho_{0}\left(\xi_{T}\right)=L^{g}
$$

Moreover, for any $T \geq 0$,

$$
\left|\rho_{0}\left(\xi_{T}\right)-L^{g}\right| \leq C\left(1+|v|^{2}\right) e^{-\hat{C}_{\eta} T}, \quad \forall v \in \mathbb{R}^{d} .
$$

Proof. Firstly, recall that, by Theorem 3.5.1 and Lemma 3.6.4.

$$
\begin{aligned}
\rho_{0}\left(\xi_{T}\right) & =Y_{0}^{T, g} \\
& =y^{T, g}(v, 0), \quad \forall v \in \mathbb{R}^{d},
\end{aligned}
$$

with the function $y^{T, g}$ given in 3.6.28. 
(i) By the statement (i) in Lemma 3.6.4, there exists a constant $C>0$ such that

$$
\left|y^{T, g}(v, 0)\right| \leq C(1+|v|), \quad \forall v \in \mathbb{R}^{d}
$$

Hence,

$$
\frac{\left|y^{T, g}(v, 0)\right|}{T} \leq \frac{C(1+|v|)}{T}, \quad \forall v \in \mathbb{R}^{d}
$$

(ii) By the statement (i) in Lemma 3.6.4 and the standard diagonal argument, there exists a sequence $\left\{T_{i}\right\}_{i=1}^{\infty}$ such that, $T_{i} \uparrow \infty$ as $i \uparrow \infty$, and

$$
\lim _{T_{i} \uparrow \infty} y^{T_{i}, g}(v, 0)=L^{g}(v), \quad \forall v \in D,
$$

where $D$ is a countable dense subset of $\mathbb{R}^{d}$, for some $L^{g}(v) \in \mathbb{R}$.

Let $v \in \mathbb{R}^{d} \backslash D$. By the denseness of $D$, there exists a sequence $\left\{v_{j}\right\}_{j=1}^{\infty} \subseteq D$ such that $v_{j} \rightarrow v$ as $j \uparrow \infty$. Define

$$
L^{g}(v) \triangleq \lim _{j \uparrow \infty} L^{g}\left(v_{j}\right)
$$

By the statement (ii) in Lemma 3.6.4, for any $i, j \in \mathbb{N}$,

$$
\left|y^{T_{i}, g}(v, 0)-y^{T_{i}, g}\left(v_{j}, 0\right)\right| \leq\left(q+\frac{C_{v}}{\gamma\left(C_{\eta}-C_{v}\right)}\right)\left|v-v_{j}\right| .
$$

Taking $i \uparrow \infty$, by 3.6.31,

$$
\left|\lim _{T_{i} \uparrow \infty} y^{T_{i}, g}(v, 0)-L^{g}\left(v_{j}\right)\right| \leq\left(q+\frac{C_{v}}{\gamma\left(C_{\eta}-C_{v}\right)}\right)\left|v-v_{j}\right| .
$$

Taking $j \uparrow \infty$, we have

$$
\lim _{T_{i} \uparrow \infty} y^{T_{i}, g}(v, 0)=L^{g}(v), \quad \forall v \in \mathbb{R}^{d} \backslash D,
$$

and hence, together with 3.6.31,

$$
\lim _{T_{i} \uparrow \infty} y^{T_{i}, g}(v, 0)=L^{g}(v), \quad \forall v \in \mathbb{R}^{d} .
$$


By the statement (iii) in Lemma 3.6.4, for any $i \in \mathbb{N}$,

$$
\left|y^{T_{i}, g}(v, 0)-y^{T_{i}, g}(\bar{v}, 0)\right| \leq C\left(1+|v|^{2}+|\bar{v}|^{2}\right) e^{-\hat{C}_{\eta} T_{i}}, \quad \forall v, \bar{v} \in \mathbb{R}^{d}
$$

Taking $i \uparrow \infty$, by 3.6.32,

$$
L^{g}(v)=L^{g}(\bar{v}), \quad \forall v, \bar{v} \in \mathbb{R}^{d} .
$$

Therefore, the limit is independent of $v$, denoted as $L^{g}$. Moreover, by the arguments on pages 394-395 in $\mathrm{Hu}$ et al. (2015), such a constant $L^{g}$ is independent of the choice of the sequence $\left\{T_{i}\right\}_{i=1}^{\infty}$. Hence,

$$
\lim _{T \uparrow \infty} y^{T, g}(v, 0)=L^{g} \quad \forall v \in \mathbb{R}^{d}
$$

Let $v \in \mathbb{R}^{d}$ and $T \geq 0$. By 3.6.30 in Lemma 3.6.4,

$$
\begin{aligned}
\left|y^{T, g}(v, 0)-L^{g}\right| & =\left|y^{T, g}(v, 0)-\lim _{T^{\prime}(\geq T) \uparrow \infty} y^{T^{\prime}, g}(v, 0)\right| \\
& =\lim _{T^{\prime}(\geq T) \uparrow \infty}\left|y^{T, g}(v, 0)-y^{T^{\prime}, g}(v, 0)\right| \\
& =\lim _{T^{\prime}(\geq T) \uparrow \infty}\left|y^{T, g}(v, 0)-\mathbb{E}^{\mathbb{Q}^{H}}\left[g\left(V_{T^{\prime}}^{0, v}\right)\right]\right| \\
& =\lim _{T^{\prime}(\geq T) \uparrow \infty}\left|y^{T, g}(v, 0)-\mathbb{E}^{\mathbb{Q}^{H}}\left[\mathbb{E}^{\mathbb{Q}^{H}}\left[g\left(V_{T^{\prime}}^{0, v}\right) \mid \mathcal{F}_{T^{\prime}-T}\right]\right]\right| \\
& =\lim _{T^{\prime}(\geq T) \uparrow \infty}\left|y^{T, g}(v, 0)-\mathbb{E}^{\mathbb{Q}^{H}}\left[y^{T^{\prime}, g}\left(V_{T^{\prime}-T}^{0, v}, T^{\prime}-T\right)\right]\right| \\
& =\lim _{T^{\prime}(\geq T) \uparrow \infty}\left|y^{T, g}(v, 0)-\mathbb{E}^{\mathbb{Q}^{H}}\left[y^{T, g}\left(V_{T^{\prime}-T}^{0, v}, 0\right)\right]\right| \\
& =\lim _{T^{\prime}(\geq T) \uparrow \infty}\left|\mathbb{E}^{\mathbb{Q}^{H}}\left[y^{T, g}(v, 0)-y^{T, g}\left(V_{T^{\prime}-T}^{0, v}, 0\right)\right]\right| \\
& \leq \lim _{T^{\prime}(\geq T) \uparrow \infty} \mathbb{E}^{\mathbb{Q}^{H}}\left[\left|y^{T, g}(v, 0)-y^{T, g}\left(V_{T^{\prime}-T}^{0, v}, 0\right)\right|\right]
\end{aligned}
$$

By the statement (iii) in Lemma 3.6.4, there exists a constant $C>0$ such that

$$
\begin{aligned}
\left|y^{T, g}(v, 0)-L^{g}\right| & \leq \lim _{T^{\prime}(\geq T) \uparrow \infty} \mathbb{E}^{\mathbb{Q}^{H}}\left[\left|y^{T, g}(v, 0)-y^{T, g}\left(V_{T^{\prime}-T}^{0, v}, 0\right)\right|\right] \\
& \leq \lim _{T^{\prime}(\geq T) \uparrow \infty} C\left(1+|v|^{2}+\mathbb{E}^{\mathbb{Q}^{H}}\left[\left|V_{T^{\prime}-T}^{0, v}\right|^{2}\right]\right) e^{-\hat{C}_{\eta} T} .
\end{aligned}
$$


By the statement (i) in Proposition 3.6.3, there exists a constant $C_{1}>0$ such that

$$
\begin{aligned}
\left|y^{T, g}(v, 0)-L^{g}\right| & \leq \lim _{T^{\prime}(\geq T) \uparrow \infty} C\left(1+|v|^{2}+\mathbb{E}^{\mathbb{Q}^{H}}\left[\left|V_{T^{\prime}-T}^{0, v}\right|^{2}\right]\right) e^{-\hat{C}_{\eta} T} \\
& \leq \lim _{T^{\prime}(\geq T) \uparrow \infty} C\left(1+|v|^{2}+C_{1}\left(1+|v|^{2}\right)\right) e^{-\hat{C}_{\eta} T} \\
& \leq \tilde{C}\left(1+|v|^{2}\right) e^{-\hat{C}_{\eta} T} .
\end{aligned}
$$

\subsubsection{Non-Markovian Exponential Forward Investment Per- formance Processes}

In this subsection, we consider the case that the exponential forward investment performance process is in the form:

$$
U(x, t)=-e^{-\gamma x+Y_{t}-\lambda t}, \quad \forall x \in \mathbb{R}, t \geq 0,
$$

where the solution triplet $(Y, Z, \lambda)$ solves the ergodic BSDE 3.4.8) with

$$
Z_{t}=z^{0}\left(V_{t}\right), \quad \forall t \geq 0
$$

for any differentiable and bounded function $z^{0}: \mathbb{R}^{d} \rightarrow \mathbb{R}^{d}$ with bounded first order derivative. For the ease of discussions, with this solution triplet $\left(Y, z^{0}, \lambda\right)$ of the ergodic BSDE (3.4.8), we rewrite the ergodic BSDE (3.6.18) here:

$$
\hat{Y}_{t}=\hat{Y}_{T^{\prime}}+\int_{t}^{T^{\prime}}\left(G\left(V_{s}, z^{0}\left(V_{s}\right), \hat{Z}_{s}\right)-\hat{\lambda}\right) d s-\int_{t}^{T^{\prime}}\left(\hat{Z}_{s}\right)^{t r} d W_{s}, \quad \forall T^{\prime} \geq 0, t \in\left[0, T^{\prime}\right] .
$$

In this case, the driver $G$ of the ergodic BSDE (3.6.34) depends on the function $z^{0}$. By the boundedness of $z_{0}$, the function $G$ satisfies the locally Lipschitz estimate (3.4.7) in $\hat{z}$ by (3.5.13). By the boundedness of the first order derivative of $z_{0}$, the

function $G$ satisfies the locally Lipschitz estimate (3.4.6) in $v$ as well. Hence, the 
existence and uniqueness results in Proposition 3.4.1 by Liang and Zariphopoulou (2017) apply to the ergodic BSDE (3.6.34). Therefore, the ergodic BSDE (3.6.34) admits a unique Markovian solution $(\hat{Y}, \hat{Z}, \lambda)$.

On the other hand, it is obvious that $(C, 0,0)$, where $C$ is a generic constant, solves the ergodic BSDE 3.6.34). Therefore, $(\hat{Y}, \hat{Z}, \lambda)=(C, 0,0)$ is the only Markovian solution to the ergodic BSDE (3.6.34). Hence, the large maturity behavior of the forward entropic risk measures is given by

$$
\hat{Y}_{0}+\hat{\lambda} T=C
$$

which is the constant. Moreover, by Lemma 4.5 in Hu et al. (2015), we can obtain similar estimates as in Lemma 3.6.4, but with different constants. Therefore, same arguments as in Theorem 3.6 .5 yield

Theorem 3.6.6. Suppose that Assumptions 3.3.1 and 3.3.2 hold, and that the exponential forward investment performance process is given by (3.6.33). Let $T \geq$ 0 and $\xi_{T} \in \mathcal{L}_{M}$. Then,

(i) there exists a constant $C>0$ such that

$$
\frac{\left|\rho_{0}\left(\xi_{T}\right)\right|}{T} \leq \frac{C(1+|v|)}{T}, \quad \forall v \in \mathbb{R}^{d}
$$

and hence,

$$
\lim _{T \uparrow \infty} \frac{\rho_{0}\left(\xi_{T}\right)}{T}=0 .
$$

(ii) there exists a constant $K^{g} \in \mathbb{R}$, independent of $v$, such that

$$
\lim _{T \uparrow \infty} \rho_{0}\left(\xi_{T}\right)=K^{g}
$$

Moreover, for any $T \geq 0$,

$$
\left|\rho_{0}\left(\xi_{T}\right)-K^{g}\right| \leq C\left(1+|v|^{2}\right) e^{-C_{\eta} T}, \quad \forall v \in \mathbb{R}^{d} .
$$




\subsection{Example and Numerical Illustrations}

In this last section of this chapter, we provide an example in which we derive explicit formula for the forward entropic risk measures. We also illustrate the numerical results for their large maturity limits.

Consider a financial market model with a single stock $S=\left\{S_{t}\right\}_{t \geq 0}$ with the dynamics depending on a single stochastic factor $V=\left\{V_{t}\right\}_{t \geq 0}$ driven by a 2dimensional Brownian motion $W=\left\{\left(W_{t}^{1}, W_{t}^{2}\right)^{t r}\right\}_{t \geq 0}$ :

$$
d S_{t}=b\left(V_{t}\right) S_{t} d t+\sigma\left(V_{t}\right) S_{t} d W_{t}^{1}, \quad \forall t \geq 0
$$

and

$$
d V_{t}=\eta\left(V_{t}\right) d t+\kappa_{1} d W_{t}^{1}+\kappa_{2} d W_{t}^{2}, \quad \forall t \geq 0,
$$

where the function $b: \mathbb{R} \rightarrow \mathbb{R}$ is uniformly bounded, the function $\sigma: \mathbb{R} \rightarrow \mathbb{R}$ is uniformly bounded and non-zero, the function $\eta: \mathbb{R} \rightarrow \mathbb{R}$ satisfies the dissipative condition 3.3.3), and $\kappa_{1}, \kappa_{2}>0$ are constants such that $\kappa_{1}^{2}+\kappa_{2}^{2}=1$. Also, assume that the market price of risk

$$
\theta(v)=\frac{b(v)}{\sigma(v)}, \quad \forall v \in \mathbb{R}^{d}
$$

is Lipschitz continuous in $v$. We also set

$$
\Pi=\mathbb{R} \times\{0\},
$$

and thus

$$
\pi_{t}^{2}=0, \quad \forall t \geq 0
$$

Therefore, the wealth process $X^{\pi}=\left\{X_{t}^{\pi}\right\}_{t \geq 0}$ in 3.3.4 satisfies

$$
d X_{t}^{\pi^{1}}=\pi_{t}^{1}\left(\theta\left(V_{t}\right) d t+d W_{t}^{1}\right), \quad \forall t \geq 0
$$

With the above setting, the driver $F$ in 3.4 .5 becomes

$$
\begin{aligned}
F\left(v,\left(z_{1}, z_{2}\right)^{t r}\right) & =-\frac{1}{2}\left|z_{1}+\theta(v)\right|^{2}+\frac{1}{2}\left|z_{1}\right|^{2}+\frac{1}{2}\left|z_{2}\right|^{2} \\
& =-\theta(v) z_{1}-\frac{1}{2}|\theta(v)|^{2}+\frac{1}{2}\left|z_{2}\right|^{2}, \quad \forall v, z_{1}, z_{2} \in \mathbb{R},
\end{aligned}
$$


while the driver $G$ in 3.5 .12 becomes

$$
\begin{aligned}
G\left(v,\left(z_{1}, z_{2}\right)^{t r},\left(\hat{z}_{1}, \hat{z}_{2}\right)^{t r}\right) & =\frac{1}{\gamma}\left(F\left(v,\left(z_{1}+\gamma \hat{z}_{1}, z_{2}+\gamma \hat{z}_{2}\right)^{t r}\right)-F\left(v,\left(z_{1}, z_{2}\right)^{t r}\right)\right) \\
& =-\theta(v) \hat{z}_{1}+z_{2} \hat{z}_{2}+\frac{\gamma}{2}\left|\hat{z}_{2}\right|^{2}, \quad \forall v, z_{1}, z_{2}, \hat{z}_{1}, \hat{z}_{2} \in \mathbb{R} .
\end{aligned}
$$

Therefore, the finite horizon BSDE (3.6.17) is given by

$$
\begin{aligned}
Y_{t}^{T, g}= & g\left(V_{T}\right)+\int_{t}^{T}\left(-\theta\left(V_{s}\right) Z_{s}^{T, g, 1}+Z_{s}^{2} Z_{s}^{T, g, 2}+\frac{\gamma}{2}\left|Z_{s}^{T, g, 2}\right|^{2}\right) d s \\
& -\int_{t}^{T} Z_{s}^{T, g, 1} d W_{s}^{1}-\int_{t}^{T} Z_{s}^{T, g, 2} d W_{s}^{2}, \quad \forall t \in[0, T] .
\end{aligned}
$$

Let $Z^{T, g}=\left\{Z_{t}^{T, g}\right\}_{t \in[0, T]}$ be a process such that

$$
Z_{t}^{T, g, 1}=\kappa_{1} Z_{t}^{T, g}, \quad \forall t \in[0, T]
$$

and

$$
Z_{t}^{T, g, 2}=\kappa_{2} Z_{t}^{T, g}, \quad \forall t \in[0, T]
$$

Then, the finite horizon BSDE becomes

$$
\begin{aligned}
Y_{t}^{T, g}= & g\left(V_{T}\right)+\int_{t}^{T}\left(\left(-\kappa_{1} \theta\left(V_{s}\right)+\kappa_{2} Z_{s}^{2}\right) Z_{s}^{T, g}+\frac{\gamma \kappa_{2}^{2}}{2}\left|Z_{s}^{T, g}\right|^{2}\right) d s \\
& -\int_{t}^{T} Z_{s}^{T, g}\left(\kappa_{1} d W_{s}^{1}+\kappa_{2} d W_{s}^{2}\right), \quad \forall t \in[0, T] .
\end{aligned}
$$

Let $\tilde{Y}^{T, g}=\left\{\tilde{Y}_{t}^{T, g}\right\}_{t \in[0, T]}$ and $\tilde{Z}^{T, g}=\left\{\tilde{Z}_{t}^{T, g}\right\}_{t \in[0, T]}$ be processes such that

$$
\tilde{Y}_{t}^{T, g}=e^{\gamma \kappa_{2}^{2} Y_{t}^{T, g}}, \quad \forall t \in[0, T]
$$

and

$$
\tilde{Z}_{t}^{T, g}=\gamma \kappa_{2}^{2} \tilde{Y}_{t}^{T, g} Z_{t}^{T, g}, \quad \forall t \in[0, T] .
$$

Then, the pair $\left(\tilde{Y}^{T, g}, \tilde{Z}^{T, g}\right)$ solves

$\tilde{Y}_{t}^{T, g}=e^{\gamma \kappa_{2}^{2} g\left(V_{T}\right)}+\int_{t}^{T} \tilde{Z}_{s}^{T, g}\left(\left(\kappa_{1} \theta\left(V_{s}\right)-\kappa_{2} Z_{s}^{2}\right) d s+\kappa_{1} d W_{s}^{1}+\kappa_{2} d W_{s}^{2}\right), \quad \forall t \in[0, T]$. 
Define the process $B=\left\{B_{t}\right\}_{t \geq 0}$ by

$$
B_{t}=\int_{0}^{t}\left(\kappa_{1} \theta\left(V_{s}\right)-\kappa_{2} Z_{s}^{2}\right) d s+\kappa_{1} d W_{s}^{1}+\kappa_{2} d W_{s}^{2}, \quad \forall t \geq 0 .
$$

Since $\theta$ and $Z^{2}$ are uniformly bounded, by Girsanov Theorem, there exists a $\mathbb{P}$ equivalent measure $\mathbb{Q}$ such that the process $B$ is a $\mathbb{Q}$-Brownian motion. Therefore, by taking the $\mathbb{Q}$-conditional expectation with respect to $\mathcal{F}_{t}$,

$$
\tilde{Y}_{t}^{T, g}=\mathbb{E}^{\mathbb{Q}}\left[e^{\gamma \kappa_{2}^{2} g\left(V_{T}\right)} \mid \mathcal{F}_{t}\right], \quad \forall t \in[0, T]
$$

and thus,

$$
Y_{t}^{T, g}=\frac{1}{\gamma \kappa_{2}^{2}} \ln \mathbb{E}^{\mathbb{Q}}\left[e^{\gamma \kappa_{2}^{2} g\left(V_{T}\right)} \mid \mathcal{F}_{t}\right], \quad \forall t \in[0, T] .
$$

Hence, by Theorem 3.5.1, the forward entropic risk measure has the closed-form representation

$$
\begin{aligned}
\rho_{t}\left(\xi_{T}\right) & =Y_{t}^{T, g} \\
& =\frac{1}{\gamma \kappa_{2}^{2}} \ln \mathbb{E}^{\mathbb{Q}}\left[e^{\gamma \kappa_{2}^{2} g\left(V_{T}\right)} \mid \mathcal{F}_{t}\right], \quad \forall t \in[0, T] .
\end{aligned}
$$

Finally, we demonstrate numerical results showing the large maturity behavior of the above forward entropic risk measure, with

$$
\begin{gathered}
\eta(v)=-\alpha v, \quad \forall v \in \mathbb{R}, \\
g(v)=\left(K_{1}-|v|\right)_{+}, \quad \forall v \in \mathbb{R}, \\
\theta(v)=\left(K_{2}-|v|\right)_{+}, \quad \forall v \in \mathbb{R},
\end{gathered}
$$

for some constants $\alpha, K_{1}, K_{2}>0$. 

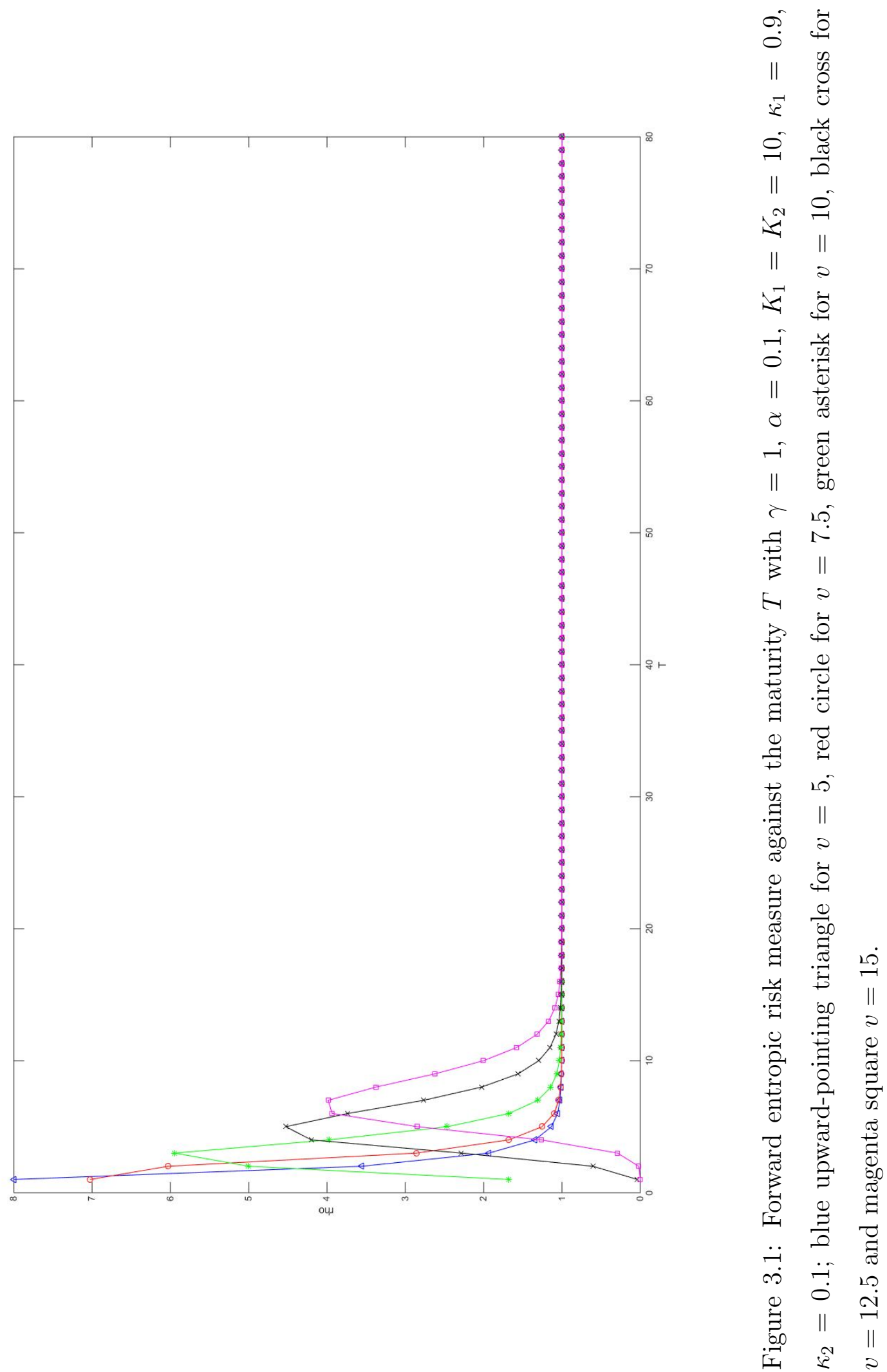

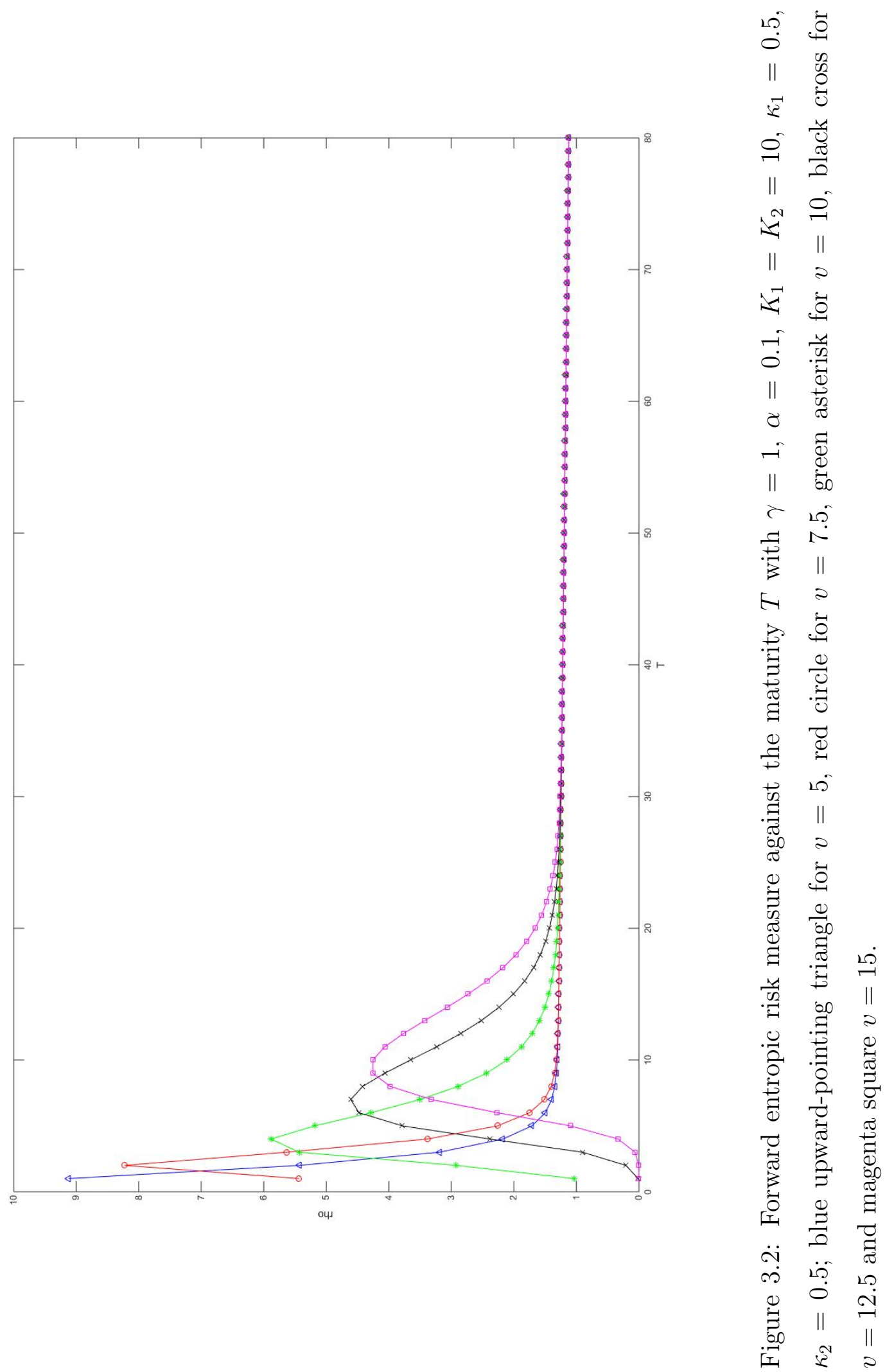

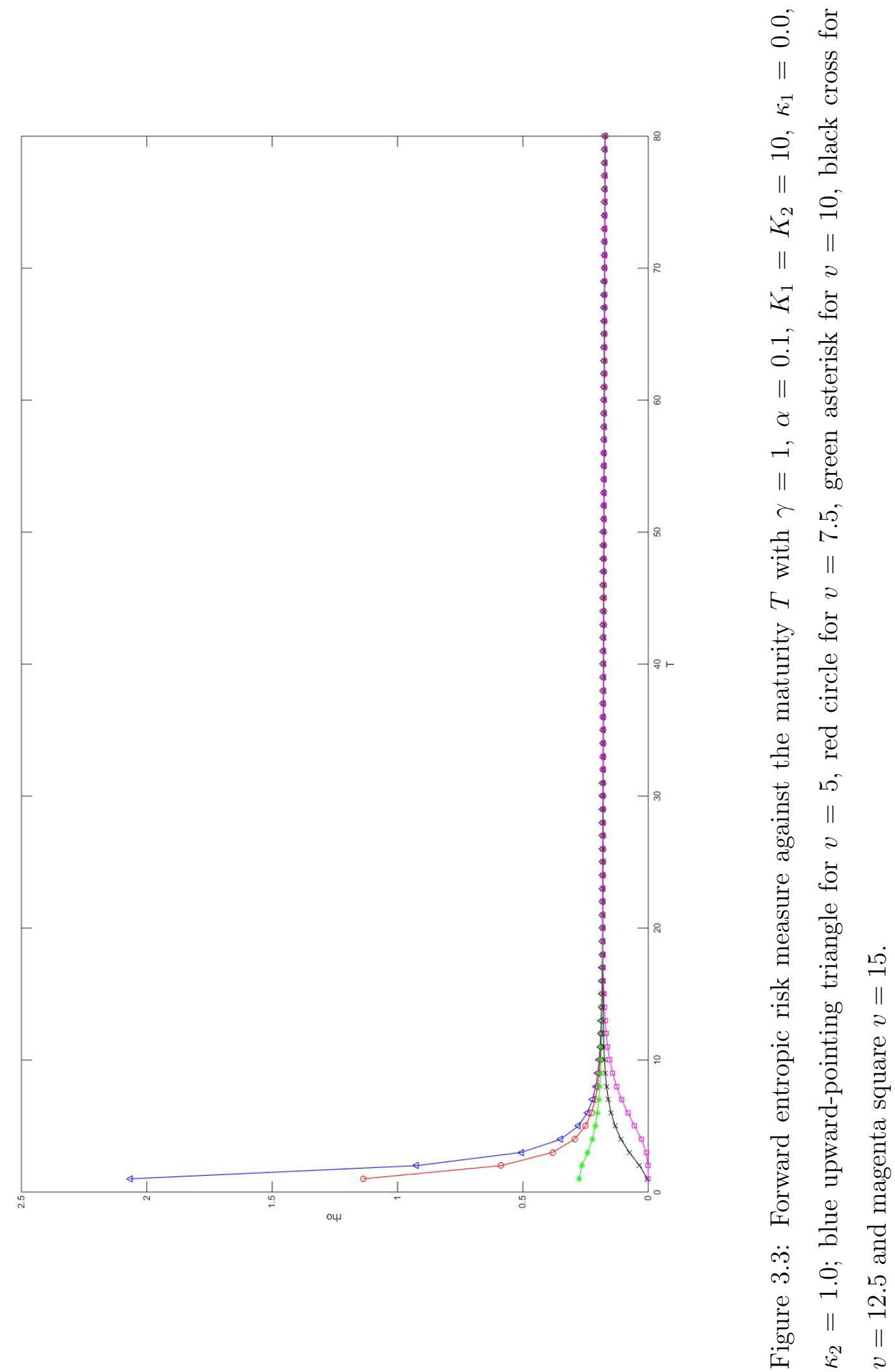


\section{Chapter 4}

\section{Forward Investment and \\ Consumption Performance}

\section{Processes}

\subsection{Motivation}

From the last chapter, we witnessed the importance of the representation for the forward investment performance processes by an ergodic or infinite horizon BSDE. In particular, that representation became the main component of the representation of the forward entropic risk measures by a finite horizon BSDE. Without the ergodic or infinite horizon BSDE representation for the forward investment performance processes, all arguments starting from Section 3.5 were vulnerable.

Hence, in this chapter, we prove a representation for the forward investment and consumption performance processes, which were introduced by Berrier and Tehranchi (2008) and Källblad (2016), by an infinite horizon BSDE. The forward investment and consumption performance processes are generalizations of the forward investment performance processes defined in the last chapter. We shall first 
recall the definition of the forward investment and consumption performance processes in the following section.

\subsection{Forward Investment and Consumption Per- formance Processes}

Let $W=\left\{W_{t}\right\}_{t \geq 0}$ be a $d$-dimensional Brownian motion on a probability space $(\Omega, \mathcal{F}, \mathbb{P})$. Denote by $\mathbb{F}=\left\{\mathcal{F}_{t}\right\}_{t \geq 0}$ the augmented filtration generated by $W$. Assume that we have a financial market environment building upon this filtered probability space $\left(\Omega, \mathcal{F}, \mathbb{F}=\left\{\mathcal{F}_{t}\right\}_{t \geq 0}, \mathbb{P}\right)$. Let $\pi$ be an investment strategy and $C$ be a consumption stream defined for all times $t \geq 0$, and denote $X^{\pi, C}=\left\{X_{t}^{\pi, C}\right\}_{t \geq 0}$ as the corresponding wealth process. We shall use the superscript $t, x$, whenever appropriate, to indicate the dependence on the initial condition $x \in \mathbb{R}$ of the wealth process at the initial time $t \geq 0$. Denote $\mathcal{A}$ as the set of admissible investment strategies and consumption streams defined for all times $t \geq 0$.

The (exponential) forward investment and consumption performance processes, which were developed by Berrier and Tehranchi (2008) and Källblad (2016), as a generalization of the (exponential) forward investment performance processes, are defined by:

Definition 4.2.1. A pair of processes

$$
\left\{\left(U(\omega, x, t), U^{c}(\omega, C, t)\right)\right\}_{\omega \in \Omega, x \in \mathbb{R}, C \in \mathbb{R}, t \geq 0}
$$

is called forward investment and consumption performance processes if they satisfy all of the following properties:

(i) for each $x \in \mathbb{R}$ and $C \in \mathbb{R},\{U(\omega, x, t)\}_{\omega \in \Omega, t \geq 0}$ and $\left\{U^{c}(\omega, C, t)\right\}_{\omega \in \Omega, t \geq 0}$ are $\mathbb{F}$-progressively measurable; 
(ii) for each $\omega \in \Omega$ and $t \geq 0,\{U(\omega, x, t)\}_{x \in \mathbb{R}}$ and $\left\{U^{c}(\omega, C, t)\right\}_{C \in \mathbb{R}}$ are strictly increasing and strictly concave;

(iii) for any $(\pi, C) \in \mathcal{A}$,

$\mathbb{E}\left[U\left(X_{s}^{\pi, C, t, x}, s\right)+\int_{t}^{s} U^{c}\left(C_{u}, u\right) d u \mid \mathcal{F}_{t}\right] \leq U(x, t), \quad \forall 0 \leq t \leq s<\infty, x \in \mathbb{R}$

and there exists an optimal pair $\left(\pi^{*}, C^{*}\right) \in \mathcal{A}$ such that

$\mathbb{E}\left[U\left(X_{s}^{\pi^{*}, C^{*}, t, x}, s\right)+\int_{t}^{s} U^{c}\left(C_{u}^{*}, u\right) d u \mid \mathcal{F}_{t}\right]=U(x, t), \quad \forall 0 \leq t \leq s<\infty, x \in \mathbb{R}$.

Moreover, a forward investment and consumption performance processes

$$
\left\{\left(U(\omega, x, t), U^{c}(\omega, C, t)\right)\right\}_{\omega \in \Omega, x \in \mathbb{R}, C \in \mathbb{R}, t \geq 0}
$$

is called an exponential forward investment and consumption performance processes, if there exist constants $\gamma, \gamma^{c}>0$ and $\mathbb{F}$-progressively measurable processes $K=\left\{K_{t}\right\}_{t \geq 0}$ and $K^{c}=\left\{K_{t}^{c}\right\}_{t \geq 0}$ such that

$$
U(x, t)=-e^{-\gamma x+K_{t}}, \quad \forall x \in \mathbb{R}, t \geq 0,
$$

and

$$
U^{c}(C, t)=-e^{-\gamma^{c} C+K_{t}^{c}}, \quad \forall C \in \mathbb{R}, t \geq 0
$$

Similar to the last chapter, we shall properly define the market model that shall be adapted since the forward investment and consumption performance processes depend on the financial market environment. We shall adapt, again, the stochastic factor market model, but, for the sake of completeness, we repeat the model in the next section. In particular, we shall consider the non-discounted stock price processes and wealth process in this chapter. 


\subsection{Stochastic Factor Market Model}

We consider a financial market model of a risk-free asset $S^{0}=\left\{S_{t}^{0}\right\}_{t \geq 0}$ with interest rate $r>0$ and $n$ risky stocks $S=\left\{\left(S_{t}^{1}, S_{t}^{2}, \ldots, S_{t}^{n}\right)^{t r}\right\}_{t \geq 0}$, with $n \leq d$, where $d$ is the dimension of the Brownian motion. The stock price processes solve, for $i=1,2, \ldots, n$,

$$
\frac{d S_{t}^{i}}{S_{t}^{i}}=b^{i}\left(V_{t}\right) d t+\sigma^{i}\left(V_{t}\right) d W_{t}
$$

while the $d$-dimensional stochastic factors $V=\left\{\left(V_{t}^{1}, V_{t}^{2}, \ldots, V_{t}^{d}\right)^{t r}\right\}_{t \geq 0}$ solves

$$
d V_{t}=\eta\left(V_{t}\right) d t+\kappa d W_{t}
$$

We assume the following hold in the remaining of this chapter.

\section{Assumption 4.3.1.}

(i) The drift and volatility coefficients $\left\{b^{i}(v)\right\}_{v \in \mathbb{R}^{d}} \in \mathbb{R}$ and $\left\{\sigma^{i}(v)\right\}_{v \in \mathbb{R}^{d}} \in \mathbb{R}^{1 \times d}$ are uniformly bounded in $v \in \mathbb{R}^{d}$;

(ii) The volatility matrix

$$
\{\sigma(v)\}_{v \in \mathbb{R}^{d}}=\left\{\left(\sigma^{1}(v), \sigma^{2}(v), \ldots, \sigma^{n}(v)\right)^{t r}\right\}_{v \in \mathbb{R}^{d}}
$$

has full row rank $n$;

(iii) The market price of risk

$$
\{\theta(v)\}_{v \in \mathbb{R}^{d}}=\left\{\sigma(v)^{t r}\left(\sigma(v) \sigma(v)^{t r}\right)^{-1}(b(v)-r \mathbb{1})\right\}_{v \in \mathbb{R}^{d}}
$$

where $\mathbb{1}=(1, \ldots, 1)^{t r} \in \mathbb{R}^{d}$, which exist by (ii), is uniformly bounded and Lipschitz continuous in $v \in \mathbb{R}^{d}$.

\section{Assumption 4.3.2.}


(i) The drift coefficient $\{\eta(v)\}_{v \in \mathbb{R}^{d}} \in \mathbb{R}^{d}$ satisfies a dissipative condition, namely, there exists a constant $C_{\eta}>0$ such that

$$
\left(\eta\left(v_{1}\right)-\eta\left(v_{2}\right)\right)^{t r}\left(v_{1}-v_{2}\right) \leq-C_{\eta}\left|v_{1}-v_{2}\right|^{2}, \quad \forall v_{1}, v_{2} \in \mathbb{R}^{d}
$$

and

$$
C_{\eta}>C_{v}>0
$$

where the positive constant $C_{v}$ is a locally Lipschitz constant for the driver in the upcoming infinite horizon BSDE (4.4.7).

(ii) The constant volatility matrix $\kappa \in \mathbb{R}^{d \times d}$ is positive definite and normalized such that $|\kappa|=1$.

Denote $\tilde{\pi}=\left\{\left(\tilde{\pi}_{t}^{1}, \tilde{\pi}_{t}^{2}, \ldots, \tilde{\pi}_{t}^{n}\right)^{t r}\right\}_{t \geq 0}$ as the amount investing into the $n$ risky assets, and $C=\left\{C_{t}\right\}_{t \geq 0}$ as the consumption rate. Then, in this stochastic factor market environment, the wealth process $X^{\tilde{\pi}, C}=\left\{X_{t}^{\tilde{\pi}, C}\right\}_{t \geq 0}$ satisfies

$$
\begin{aligned}
d X_{t}^{\tilde{\pi}, C} & =\left(X_{t}^{\tilde{\pi}, C}-\sum_{i=1}^{n} \tilde{\pi}_{t}^{i}\right) r d t+\sum_{i=1}^{n} \tilde{\pi}_{t}^{i} \frac{d S_{t}^{i}}{S_{t}^{i}}-C_{t} d t \\
& =X_{t}^{\tilde{\pi}, C} r d t+\tilde{\pi}^{t r} \sigma\left(V_{t}\right)\left(\theta\left(V_{t}\right) d t+d W_{t}\right)-C_{t} d t, \quad \forall t \geq 0 .
\end{aligned}
$$

Denote $\pi=\left\{\left(\pi_{t}^{1}, \pi_{t}^{2}, \ldots, \pi_{t}^{n}\right)^{t r}\right\}_{t \geq 0}$ as the investment strategies rescaled by the volatility:

$$
\pi_{t}^{t r}=\tilde{\pi}_{t}^{t r} \sigma\left(V_{t}\right), \quad \forall t \geq 0
$$

Therefore, the wealth process $X^{\pi, C}=\left\{X_{t}^{\pi, C}\right\}_{t \geq 0}$ satisfies

$$
d X_{t}^{\pi, C}=\left(X_{t}^{\pi, C} r+\pi_{t}^{t r} \theta\left(V_{t}\right)-C_{t}\right) d t+\pi_{t}^{t r} d W_{t}, \quad \forall t \geq 0
$$

For any $t \geq 0$, denote $\mathcal{A}[0, t]$ as the set of admissible investment and consump- 
tion strategies for times $s \in[0, t]$ :

$$
\begin{aligned}
& \mathcal{A}[0, t]=\{\left(\pi=\left\{\pi_{s}\right\}_{s \in[0, t]}, C=\left\{C_{s}\right\}_{s \in[0, t]}\right): \pi \in \mathcal{L}_{B M O}^{2}[0, t] ; \pi_{s} \in \Pi, \forall s \in[0, t] ; \\
& C \text { is } \mathbb{F} \text {-progessively measureable; } \int_{0}^{t}\left|C_{s}\right| d s<\infty \mathbb{P} \text {-a.s.; } \mathbb{E}\left[\int_{0}^{t} e^{-\gamma C_{s}} d s\right]<\infty ; \\
&\left.C_{s} \in \mathbb{R}, \forall s \in[0, t] ; \text { and }\left\{e^{-\gamma r X_{s}^{\pi, C}}\right\}_{s \in[0, t]} \text { is of class }(D)\right\},
\end{aligned}
$$

where

$$
\begin{aligned}
\mathcal{L}_{B M O}^{2}[0, t]=\left\{\pi=\left\{\pi_{s}\right\}_{s \in[0, t]}:\right. & \pi \text { is } \mathbb{F} \text {-progessively measureable and } \\
& \left.\begin{array}{l}
\text { ess sup } \\
\tau \in \mathcal{T}[0, t]
\end{array}\left[\int_{\tau}^{t}\left|\pi_{s}\right|^{2} d s \mid \mathcal{F}_{\tau}\right]<\infty\right\}
\end{aligned}
$$

$\mathcal{T}[0, t]$ is the set of $\mathbb{F}$-stopping time $\tau \in[0, t], \Pi$ is a closed and convex subset of $\mathbb{R}^{d}$, and $\gamma>0$. Then, the set $\mathcal{A}$ of admissible investment strategies defined for times $t \geq 0$ is defined by

$$
\mathcal{A}=\cup_{t \geq 0} \mathcal{A}[0, t]
$$

\subsection{Infinite Horizon BSDE Representation of Ex- ponential Forward Investment and Consump- tion Performance Processes}

In this section, we provide and prove the representation of an exponential forward investment and consumption performance processes.

Define a function $\mathbb{R}^{d} \times \mathbb{R} \times \mathbb{R}^{d} \rightarrow \mathbb{R}$ by

$$
\begin{array}{r}
F(v, y, z)=\frac{\gamma^{2} r^{2}}{2} \operatorname{dist}^{2}\left\{\Pi, \frac{z+\theta(v)}{\gamma r}\right\}-\frac{1}{2}|z+\theta(v)|^{2}+\frac{1}{2}|z|^{2}-r y-r(\ln r-1), \\
\forall v \in \mathbb{R}^{d}, y \in \mathbb{R}, z \in \mathbb{R}^{d} .
\end{array}
$$


By Assumption 4.3.1 and the Lipschitz continuous property of the distance function, there exist constants $C_{v}>0, C_{z}>0$, and $K>0$ such that, for any $v_{1}, v_{2}, z_{1}, z_{2} \in \mathbb{R}^{d}$ and $y_{1}, y_{2} \in \mathbb{R}$

$$
\begin{array}{r}
\left|F\left(v_{1}, y_{1}, z_{1}\right)-F\left(v_{2}, y_{1}, z_{1}\right)\right| \leq C_{v}\left(1+\left|z_{1}\right|\right)\left|v_{1}-v_{2}\right|, \\
\left|F\left(v_{1}, y_{1}, z_{1}\right)-F\left(v_{1}, y_{1}, z_{2}\right)\right| \leq C_{z}\left(1+\left|z_{1}\right|+\left|z_{2}\right|\right)\left|z_{1}-z_{2}\right|, \\
\left(y_{1}-y_{2}\right)\left(F\left(v_{1}, y_{1}, z_{1}\right)-F\left(v_{1}, y_{2}, z_{1}\right)\right) \leq-r\left(y_{1}-y_{2}\right)^{2},
\end{array}
$$

and

$$
\left|F\left(v_{1}, 0,0\right)\right| \leq K
$$

Proposition 4.4.1. Suppose that Assumptions 4.3.1 and 4.3.2 hold. Then the infinite horizon BSDE

$$
d Y_{t}=-F\left(V_{t}, Y_{t}, Z_{t}\right) d t+Z_{t}^{t r} d W_{t}, \quad \forall t \geq 0
$$

admits a unique Markovian solution $\left(Y=\left\{Y_{t}\right\}_{t \geq 0}, Z=\left\{Z_{t}\right\}_{t \geq 0}\right)$. Specifically, there exist a function $y: \mathbb{R}^{d} \rightarrow \mathbb{R}$ such that

$$
Y_{t}=y\left(V_{t}\right), \quad \forall t \geq 0
$$

and a function $z: \mathbb{R}^{d} \rightarrow \mathbb{R}^{d}$ such that

$$
Z_{t}=z\left(V_{t}\right), \quad \forall t \geq 0
$$

The functions $y$ and $z$ are uniformly bounded:

$$
|y(v)| \leq \frac{K}{r}, \quad \forall v \in \mathbb{R}^{d}
$$

and

$$
|z(v)| \leq \frac{C_{v}}{C_{\eta}-C_{v}}, \quad \forall v \in \mathbb{R}^{d}
$$

Proof. By 4.4.3 -4.4.6), the proof follows by the arguments in Briand and $\mathrm{Hu}$ (1998) and Liang and Zariphopoulou (2017). 
Theorem 4.4.2. Suppose that Assumptions 4.3.1 and 4.3.2 hold. Let $(Y, Z)$ be a solution to Equation (4.4.7) such that both $Y$ and $Z$ are uniformly bounded. Then the pair of processes

$$
\left\{\left(U(\omega, x, t), U^{c}(\omega, C, t)\right)\right\}_{\omega \in \Omega, x \in \mathbb{R}, C \in \mathbb{R}, t \geq 0}
$$

defined by

$$
U(x, t)=-e^{-\gamma r x+Y_{t}}, \quad \forall x \in \mathbb{R}, t \geq 0
$$

and

$$
U^{c}(C, t)=-e^{-\gamma C}, \quad \forall C \in \mathbb{R}, t \geq 0
$$

where $\gamma>0$, is exponential forward investment and consumption performance processes, with the associated optimal investment strategy $\left(\pi^{*}, C^{*}\right)$ defined by

$$
\pi_{t}^{*}=\mathcal{P}_{\Pi}\left(\frac{Z_{t}+\theta\left(V_{t}\right)}{\gamma r}\right), \quad \forall t \geq 0
$$

and

$$
C_{t}^{*}=r X_{t}^{\pi^{*}, C^{*}}-\frac{Y_{t}+\ln r}{\gamma}, \quad \forall t \geq 0
$$

Proof. We only need to check the condition (iii) in Definition 4.2.1 as other conditions in Definition 4.2.1 are obviously true.

Let $t \geq 0, x \in \mathbb{R}$ and $(\pi, C) \in \mathcal{A}$. Define a process $R^{\pi, C}=\left\{R_{s}^{\pi, C}\right\}_{s \geq t}$ by

$$
R_{s}^{\pi, C}=-e^{-\gamma r X_{s}^{\pi, C, t, x}+Y_{s}}+\int_{t}^{s}\left(-e^{-\gamma C_{u}}\right) d u, \quad \forall s \geq t .
$$

Then, by 4.3.1 and 4.4.7), $R^{\pi, C}$ satisfies

$$
\begin{aligned}
& d R_{s}^{\pi, C}=e^{-\gamma r X_{s}^{\pi, C, t, x}+Y_{s}}\left[\left(\left(-\frac{1}{2} \gamma^{2} r^{2}\left|\pi_{s}\right|^{2}+\gamma r \pi_{s}^{t r}\left(\theta\left(V_{s}\right)+Z_{s}\right)\right)\right.\right. \\
& +\left(-e^{\gamma r X_{s}^{\pi, C, t, x}-Y_{s}} e^{-\gamma C_{s}}-\gamma r C_{s}\right) \\
& \left.+\gamma r^{2} X_{s}^{\pi, C, t, x}-\frac{1}{2}\left|Z_{s}\right|^{2}+F\left(V_{s}, Y_{s}, Z_{s}\right)\right) d s \\
& \left.+\left(\gamma r \pi_{s}-Z_{s}\right)^{t r} d W_{s}\right], \quad \forall s \geq t
\end{aligned}
$$


Note that

$$
\begin{aligned}
& -\frac{1}{2} \gamma^{2} r^{2}\left|\pi_{s}\right|^{2}+\gamma r \pi_{s}^{t r}\left(\theta\left(V_{s}\right)+Z_{s}\right) \\
= & -\frac{1}{2} \gamma^{2} r^{2}\left(\left|\pi_{s}\right|^{2}-2 \pi_{s}^{t r} \frac{\theta\left(V_{s}\right)+Z_{s}}{\gamma r}\right) \\
= & -\frac{1}{2} \gamma^{2} r^{2}\left(\left|\pi_{s}-\frac{\theta\left(V_{s}\right)+Z_{s}}{\gamma r}\right|^{2}-\frac{\left|\theta\left(V_{s}\right)+Z_{s}\right|^{2}}{\gamma^{2} r^{2}}\right) \\
= & -\frac{1}{2} \gamma^{2} r^{2}\left|\pi_{s}-\frac{\theta\left(V_{s}\right)+Z_{s}}{\gamma r}\right|^{2}+\frac{1}{2}\left|\theta\left(V_{s}\right)+Z_{s}\right|^{2} \\
\leq & -\frac{1}{2} \gamma^{2} r^{2} \operatorname{dist}^{2}\left\{\Pi, \frac{\theta\left(V_{s}\right)+Z_{s}}{\gamma r}\right\}+\frac{1}{2}\left|\theta\left(V_{s}\right)+Z_{s}\right|^{2}, \quad \forall s \geq t,
\end{aligned}
$$

with the equality holds with

$$
\pi_{s}^{*}=\mathcal{P}_{\Pi}\left(\frac{Z_{s}+\theta\left(V_{s}\right)}{\gamma r}\right), \quad \forall s \geq t
$$

Since the function $h: \mathbb{R} \rightarrow \mathbb{R}$ given by

$$
h(z)=-e^{\gamma r X_{s}^{\pi, C, t, x}-Y_{s}} e^{-\gamma z}-\gamma r z, \quad \forall z \in \mathbb{R},
$$

is strictly concave,

$$
-e^{\gamma r X_{s}^{\pi, C, t, x}-Y_{s}} e^{-\gamma C_{s}}-\gamma r C_{s} \leq r(\ln r-1)+r Y_{s}-\gamma r^{2} X_{s}^{\pi, C, t, x}, \quad \forall s \geq t
$$

with the equality holds with

$$
C_{s}^{*}=r X_{s}^{\pi, C^{*}, t, x}-\frac{Y_{s}+\ln r}{\gamma}, \quad \forall s \geq t .
$$


Therefore, by the form of the driver $F$ in 4.4 .2 ,

$$
\begin{aligned}
& d R_{s}^{\pi, C}=e^{-\gamma r X_{s}^{\pi, C, t, x}+Y_{s}}\left[\left(\left(-\frac{1}{2} \gamma^{2} r^{2}\left|\pi_{s}\right|^{2}+\gamma r \pi_{s}^{t r}\left(\theta\left(V_{s}\right)+Z_{s}\right)\right)\right.\right. \\
& +\left(-e^{\gamma r X_{s}^{\pi, C, t, x}-Y_{s}} e^{-\gamma C_{s}}-\gamma r C_{s}\right) \\
& \left.+\gamma r^{2} X_{s}^{\pi, C, t, x}-\frac{1}{2}\left|Z_{s}\right|^{2}+F\left(V_{s}, Y_{s}, Z_{s}\right)\right) d s \\
& \left.+\left(\gamma r \pi_{s}-Z_{s}\right)^{t r} d W_{s}\right] \\
& \leq e^{-\gamma r X_{s}^{\pi, C, t, x}+Y_{s}}\left[\left(\left(-\frac{1}{2} \gamma^{2} r^{2} \operatorname{dist}^{2}\left\{\Pi, \frac{\theta\left(V_{s}\right)+Z_{s}}{\gamma r}\right\}+\frac{1}{2}\left|\theta\left(V_{s}\right)+Z_{s}\right|^{2}\right)\right.\right. \\
& +\left(r(\ln r-1)+r Y_{s}-\gamma r^{2} X_{s}^{\pi, C, t, x}\right) \\
& \left.+\gamma r^{2} X_{s}^{\pi, C, t, x}-\frac{1}{2}\left|Z_{s}\right|^{2}+F\left(V_{s}, Y_{s}, Z_{s}\right)\right) d s \\
& \left.+\left(\gamma r \pi_{s}-Z_{s}\right)^{t r} d W_{s}\right] \\
& =e^{-\gamma r X_{s}^{\pi, C, t, x}+Y_{s}}\left(\gamma r \pi_{s}-Z_{s}\right)^{t r} d W_{s}, \quad \forall s \geq t .
\end{aligned}
$$

By (4.4.8), we deduce that, for any $(\pi, C) \in \mathcal{A}, R^{\pi, C}$ is a local supermartingale. By the conditions in the admissible set of investment and consumption strategies, and the assumption that $Y$ is uniformly bounded, for any $(\pi, C) \in \mathcal{A}, R^{\pi, C}$ is of class $(D)$, and hence a proper supermartingale, i.e.,

$$
\mathbb{E}\left[R_{s}^{\pi, C} \mid \mathcal{F}_{t}\right] \leq R_{t}^{\pi, C}, \quad \forall 0 \leq t \leq s<\infty, x \in \mathbb{R}
$$

Moreover, by 4.4.8), we deduce that there exists a pair $\left(\pi^{*}, C^{*}\right) \in \mathcal{A}$ which is given by

$$
\pi_{t}^{*}=\mathcal{P}_{\Pi}\left(\frac{Z_{t}+\theta\left(V_{t}\right)}{\gamma r}\right), \quad \forall t \geq 0,
$$

and

$$
C_{t}^{*}=r X_{t}^{\pi^{*}, C^{*}}-\frac{Y_{t}+\ln r}{\gamma}, \quad \forall t \geq 0
$$

such that $R^{\pi^{*}, C^{*}}$ is a local martingale. By the conditions in the admissible set of investment and consumption strategies, and the assumption that $Y$ is uniformly 
bounded, $R^{\pi^{*}, C^{*}}$ is a proper martingale, i.e.,

$$
\mathbb{E}\left[R_{s}^{\pi^{*}, C^{*}} \mid \mathcal{F}_{t}\right]=R_{t}^{\pi^{*}, C^{*}}, \quad \forall 0 \leq t \leq s<\infty, x \in \mathbb{R}
$$

The proof that $\left(\pi^{*}, C^{*}\right) \in \mathcal{A}$ follows by similar arguments as in Cheridito and $\mathrm{Hu}$ (2011).

Before closing this chapter, we make a remark that, by Proposition 4.4.1, there exists at least one solution $(Y, Z)$ of the infinite horizon BSDE 4.4.7) which are uniformly bounded, which justifies the validity of the condition that the solution $(Y, Z)$ is uniformly bounded in Theorem 4.4.2. 


\section{Bibliography}

Arrow, K. J. (1974). Optimal insurance and generalized deductibles. Scandinavian Actuarial Journal, 1:1-42.

Artzner, P., Delbaen, F., Eber, J. M., and Heath, D. (1999). Coherent measures of risk. Mathematical Finance, 9(3):203-228.

Asimit, A. V., Badescu, A. M., and Cheung, K. C. (2013). Optimal reinsurance in the presence of counterparty default risk. Insurance: Mathematics and Economics, 53(3):690-697.

Balbás, A., Balbás, B., and Heras, A. (2009). Optimal reinsurance with general risk measures. Insurance: Mathematics and Economics, 44:374-384.

Barbu, V. and Precupanu, T. (2012). Convexity and Optimization in Banach Spaces. Springer.

Bernard, C., He, X., Yan, J. A., and Zhou, X. Y. (2015). Optimal insurance design under rank-dependent expected utility. Mathematical Finance, 25(1):154-186.

Berrier, F. P. Y. S. and Tehranchi, M. R. (2008). Forward utility of investment and consumption. Working Papers.

Billingsley, P. (2009). Convergence of Probability Measures, volume 493. WileyInterscience. 
Boonen, T. J., Tan, K. S., and Zhuang, S. C. (2016). The role of a representative reinsurer in optimal reinsurance. Insurance: Mathematics and Economics, 70:196-204.

Borch, K. (1960). An attempt to determine the optimum amount of stop loss reinsurance. In The Sixteenth International Congress of Actuaries.

Briand, P. and Hu, Y. (1998). Stability of BSDEs with random terminal time and homogenization of semilinear elliptic pdes. Journal of Functional Analysis, 155:455-494.

Bühlmann, H. (1970). Mathematical Methods in Risk Theory. Berlin: Springer.

Cai, J., Lemieux, C., and Liu, F. (2016). Optimal reinsurance from the perspective of both an insurer and a reinsurer. ASTIN Bulletin, 46(3):815-849.

Cai, J. and Tan, K. S. (2007). Optimal retention for a stop-loss reinsurance under the VaR and CTE risk measures. ASTIN Bulletin, 37:93-112.

Cai, J., Tan, K. S., Weng, C., and Zhang, Y. (2008). Optimal reinsurance under VaR and CTE risk measures. Insurance: Mathematics and Economics, 43:185196.

Cai, J. and Wei, W. (2012). Optimal reinsurance with positively dependent risks. Insurance: Mathematics and Economics, 50(1):57-63.

Cai, J. and Weng, C. (2016). Optimal reinsurance with expectile. Scandinavian Actuarial Journal, pages 624-645.

Carmona, R. E. (2009). Indifference Pricing: Theory and Applications. Princeton University Press.

Cheridito, P. and Hu, Y. (2011). Optimal consumption and investment in incomplete markets with general constraints. Stochastics and Dynamics, 11:283-299. 
Cheung, K. C. (2010). Optimal reinsurance revisited-geometric approach. ASTIN Bulletin, 40(1):221-239.

Cheung, K. C., Chong, W. F., and Lo, A. (2017). Optimal reinsurance under law-invariant coherent risk measures. Preprint.

Cheung, K. C., Chong, W. F., and Yam, S. C. P. (2015a). Convex ordering for insurance preferences. Insurance: Mathematics and Economics.

Cheung, K. C., Chong, W. F., and Yam, S. C. P. (2015b). The optimal insurance under disappointment theories. Insurance: Mathematics and Economics.

Cheung, K. C. and Lo, A. (2017). Characterizations of optimal reinsurance treaties: A cost-benefit approach. Scandinavian Actuarial Journal.

Cheung, K. C., Sung, K. C. J., and Yam, S. C. P. (2013). Risk-minimizing reinsurance protection for multivariate risks. Journal of Risk and Insurance, 81(1):219-236.

Cheung, K. C., Sung, K. C. J., Yam, S. C. P., and Yung, S. P. (2014). Optimal reinsurance under general law-invariant risk measures. Scandinavian Actuarial Journal, pages $72-91$.

Chi, Y. (2012a). Optimal reinsurance under variance related premium principles. Insurance: Mathematics and Economics, 51(2):310-321.

Chi, Y. (2012b). Reinsurance arrangements minimizing the risk-adjusted value of an insurer's liability. ASTIN Bulletin, 42(2):529-557.

Chi, Y. and Tan, K. S. (2013). Optimal reinsurance with general premium principles. Insurance: Mathematics and Economics, 52(2):180-189.

Chi, Y. and Weng, C. (2013). Optimal reinsurance subject to Vajda condition. Insurance: Mathematics and Economics, 53(1):179-189. 
Chong, W. F., Hu, Y., Liang, G., and Zariphopoulou, T. (2017). An ergodic BSDE approach to forward entropic risk measures: representation and large-maturity behavior. Revised and resubmitted to Finance and Stochastics. arXiv:1607.02289.

Chong, W. F. and Liang, G. (2017). Representation of forward investment and consumption performance process and its large time behavior. Working Papers.

Coquet, F., Hu, Y., Memin, J., and Peng, S. (2002). Filtratio-consistent nonlinear expectations and related g-expectations. Probability Theory and Related Fields, $123: 1-27$.

Cui, W., Yang, J., and Wu, L. (2013). Optimal reinsurance minimizing the distortion risk measure under general reinsurance premium principles. Insurance: Mathematics and Economics, 53:74-85.

Denuit, M., Dhaene, J., Goovaerts, M., and Kaas, R. (2005). Actuarial Theory for Dependent Risks: Measures, Orders and Models. John Wiley \& Sons.

Detlefsen, K. and Scandolo, G. (2005). Conditional and dynamic convex risk measures. Finance and Stochastics, 9:539-561.

Dhaene, J., Vanduffel, S., Goovaerts, M. J., Kaas, R., Tang, Q., and Vyncke, D. (2006). Risk measures and comonotonicity: a review. Stochastic Models, 22(4):573-606.

El Karoui, N. and Rouge, R. (2000). Pricing via utility maximization and entropy. Mathematical Finance, 10(2):259-276.

Fabian, M., Habala, P., Hajek, P., Montesinos Santalucia, V., Pelant, J., and Zizler, V. (2001). Functional Analysis and Infinite-Dimensional Geometry. Springer. 
Fleming, W. H. and McEneaney, W. M. (1995). Risk-sensitive control on an infinite time horizon. SIAM Journal on Control and Optimization, 33(6):18811915.

Föllmer, H. and Schied, A. (2002). Convex measures of risk and trading constraints. Stochastic Analysis and Applications to Finance, 6(4):429-447.

Föllmer, H. and Schied, A. (2010). Stochastic Finance: An Introduction in Discrete Time. Berlin: de Gruyter.

Frittelli, M. and Gianin, E. R. (2002). Putting order in risk measures. Journal of Banking \& Finance, 26(7):1473-1486.

Gerber, H. U. (1979). An Introduction to Mathematical Risk Theory. S. S. Huebner Foundation, R. D. Irwin Inc. Homeward Illinois.

Gollier, C. and Schlesinger, H. (1996). Arrow's theorem on the optimality of deductibles: a stochastic dominance approach. Economic Theory, 7(2):359363.

Goovaerts, M. J., De Vyler, F., and Haezendonck, J. (1984). Insurance Premiums: Theory and Applications. North-Holland: Elsevier Science Ltd.

Henderson, V. (2002). Valuation of claims on non-traded assets using utility maximization. Mathematical Finance, 12(4):351-373.

Henderson, V. and Liang, G. (2014). Pseudo linear pricing rule for utility indifference valuation. Finance and Stochastics, 18(3):593-615.

Hodges, S. and Neuberger, A. (1989). Optimal replication of contingent claims under transactions costs. Review of Futures Markets, 8:222-239.

Hu, Y., Imkeller, P., and Müller, M. (2005). Utility maximization in incomplete markets. Annals of Applied Probability, 15:1691-1712. 
Hu, Y., Madec, P., and Richou, A. (2015). A probabilistic approach to large time behavior of mild solutions of HJB equations in infinite dimension. SIAM Journal on Control and Optimization, 53(1):378-398.

Jobert, A. and Rogers, L. C. (2008). Valuations and dynamic convex risk measures. Mathematical Finance, 18(1):1-22.

Kaas, R., Goovaerts, M., Dhaene, J., and Denuit, M. (2008). Modern Actuarial Risk Theory: Using R. Springer Science \& Business Media.

Kahneman, D. and Tversky, A. (1992). Advances in prospect theory: Cumulative representation of uncertainty. Journal of Risk and Uncertainty, 5(4):297-323.

Källblad, S. (2016). Investment and consumption with forward criteria and Black's inverse investment problem. Working Papers.

Kaluszka, M. (2001). Optimal reinsurance under mean-variance premium principles. Insurance: Mathematics and Economics, 28:61-67.

Kaluszka, M. (2004). An extension of Arrow's result on optimality of a stop loss contract. Insurance: Mathematics and Economics, 35:527-536.

Kaluszka, M. (2005). Optimal reinsurance under convex principles of premium calculation. Insurance: Mathematics and Economics, 36:375-398.

Kaluszka, M. and Okolewski, A. (2008). An extension of Arrow's result on optimal reinsurance contract. Journal of Risk and Insurance, 75(2):275-288.

Karlin, S. and Novikoff, A. (1963). Generalized convex inequalities. Pacific Journal of Mathematics, 13(4):1251-1279.

Klenke, A. (2014). Probability Theory - A Comprehensive Course. Springer.

Klöppel, S. and Schweizer, M. (2007). Dynamic indifference valuation via convex risk measures. Mathematical Finance, 17(4):599-627. 
Kobylanski, M. (2000). Backward stochastic differential equations and partial differential equations with quadratic growth. Annals of Probability, 28:558-602.

Liang, G. and Zariphopoulou, T. (2017). Representation of homothetic forward performance processes in stochastic factor models via ergodic and infinite horizon BSDE. SIAM Journal on Financial Mathematics. Accepted.

Lo, A. (2017). A Neyman-Pearson perspective on optimal reinsurance with constraints. ASTIN Bulletin, 47(2).

Mania, M. and Schweizer, M. (2005). Dynamic exponential utility indifference valuation. Annals of Applied Probability, 15:2113-2143.

Musiela, M. and Zariphopoulou, T. (2004). An example of indifference prices under exponential preferences. Finance and Stochastics, 8:229-239.

Musiela, M. and Zariphopoulou, T. (2005). The backward and forward dynamic utilities and the associated pricing systems: Case study of the binomial model. Preprint.

Musiela, M. and Zariphopoulou, T. (2007). Investment and valuation under backward and forward dynamic exponential utilities in a stochastic factor model. Advances in Mathematical Finance, pages 303-334.

Musiela, M. and Zariphopoulou, T. (2008). Optimal asset allocation under forward exponential performance criteria. In Markov Processes and Related Topics: A Festschrift for Thomas G. Kurtz, Lecture Notes-Monograph Series. Institute for Mathematical Statistics.

Musiela, M. and Zariphopoulou, T. (2009a). Derivative pricing, investment management and the term structure of exponential utilities: The case of binomial model. In Indifference Pricing. Princeton University Press. 
Musiela, M. and Zariphopoulou, T. (2009b). Portfolio choice under dynamic investment performance criteria. Quantitative Finance, 9(2):161-170.

Musiela, M. and Zariphopoulou, T. (2010a). Portfolio choice under space-time monotone performance criteria. SIAM Journal of Financial Mathematics, $1: 326-365$.

Musiela, M. and Zariphopoulou, T. (2010b). Stochastic partial differential equations and portfolio choice. In Contemporary Quantitative Finance. Springer.

Ohlin, J. (1969). On a class of measures of dispersion with application to optimal reinsurance. ASTIN Bulletin, 5(2):249-266.

Pardoux, E. and Peng, S. (1990). Adapted solution of a backward stochastic differential equation. Systems \& Control Letters, 14:55-61.

Peng, S. (2004). Nonlinear expectations, nonlinear evaluations and risk measures. In Stochastic Methods in Finance. Springer Berlin Heidelberg.

Promislow, S. D. and Young, V. R. (2005). Unifying framework for optimal insurance. Insurance: Mathematics and Economics, 36(3):347-364.

Raviv, A. (1979). The design of an optimal insurance policy. American Economic Review, 69:84-96.

Riedel, F. (2004). Dynamic coherent risk measures. Stochastic Processes and their Applications, 112(2):185-200.

Stoyan, D. (1983). Comparison Methods for Queues and Other Stochastic Models. John Wiley \& Sons.

Sung, K. C. J., Yam, S. C. P., Yung, S. P., and Zhou, J. H. (2011). Behavioral optimal insurance. Insurance: Mathematics and Economics, 49(3):418-428. 
Tan, K. S., Weng, C., and Zhang, Y. (2009). VaR and CTE criteria for optimal quota-share and stop-loss reinsurance. North American Actuarial Journal, 13(4):450-482.

Tan, K. S., Weng, C., and Zhang, Y. (2011). Optimality of general reinsurance contracts under CTE risk measure. Insurance: Mathematics and Economics, 49(2):175-187.

Taylor, J. M. (1983). Comparisons of certain distribution functions. Statistics: A Journal of Theoretical and Applied Statistics, 14(3):397-408.

Wang, S. S., Young, V. R., and Panjer, H. H. (1997). Axiomatic characterization of insurance prices. Insurance: Mathematics and Economics, 21(2):173-183.

Young, V. R. (1999). Optimal insurance under Wang's premium principle. Insurance: Mathematics and Economics, 25(2):109-122.

Zariphopoulou, T. and Žitković, G. (2010). Maturity-independent risk measures. SIAM Journal of Financial Mathematics, 1:266-288.

Zheng, Y. and Cui, W. (2014). Optimal reinsurance with premium constraint under distortion risk measures. Insurance: Mathematics and Economics, 59:109120.

Zhuang, S. C., Boonen, T. J., Tan, K. S., and Xu, Z. Q. (2016). Optimal insurance in the presence of reinsurance. Scandinavian Actuarial Journal. 